\title{
Classical and quantum results on logarithmic terms in the soft theorem in four dimensions
}

\author{
Biswajit Sahoo and Ashoke Sen \\ Harish-Chandra Research Institute, HBNI, \\ Chhatnag Road, Jhusi, Allahabad 211019, India \\ E-mail: biswajitsahoo@hri.res.in, sen@hri.res.in
}

ABSTRACT: We explore the logarithmic terms in the soft theorem in four dimensions by analyzing classical scattering with generic incoming and outgoing states and one loop quantum scattering amplitudes. The classical and quantum results are consistent with each other. Although most of our analysis in quantum theory is carried out for one loop amplitudes in a theory of (charged) scalars interacting via gravitational and electromagnetic interactions, we expect the results to be valid more generally.

KEYwords: Gauge Symmetry, Scattering Amplitudes

ARXIV EPRINT: 1808.03288 


\section{Contents}

1 Introduction 1

2 Summary and analysis of the results 3

2.1 Summary of the results 4

2.2 Discussion of results 6

$\begin{array}{ll}2.3 \text { Special cases } & 10\end{array}$

3 Classical analysis $\quad \mathbf{1 2}$

$\begin{array}{lll}3.1 & \text { Effect of electromagnetic interactions } & 13\end{array}$

$\begin{array}{lll}3.2 & \text { Effect of gravitational interactions } & 15\end{array}$

$\begin{array}{lll}3.3 & \text { Effect of electromagnetic and gravitational interactions } & 17\end{array}$

4 How to treat momentum conservation and infrared divergences $\quad 18$

5 Soft photon theorem in scalar QED $\quad 20$

6 Soft graviton theorem in gravitational scattering 28

$\begin{array}{lll}7 & \text { Generalizations } & \mathbf{3 8}\end{array}$

$\begin{array}{lll}7.1 & \text { Soft graviton theorem } & 39\end{array}$

$\begin{array}{lll}7.2 & \text { Soft photon theorem } & 41\end{array}$

\section{Introduction}

Soft theorems [1-12] have been analyzed recently from different perspectives, both using asymptotic symmetries [13-36] and also by direct analysis of amplitudes in field theory and string theory [37-87]. There are general arguments establishing their validity in any spacetime dimensions in any theory as long as one maintains the relevant gauge symmetries - general coordinate invariance for soft graviton theorem and $\mathrm{U}(1)$ gauge invariance for soft photon theorem [81, 83, 84]. However these arguments break down in four space-time dimensions due to infrared divergences [41] where more care may be needed [27, 44]. Indeed, since the S-matrix itself is infrared divergent, it is not a priori clear how to interpret a relation whose both sides are divergent.

Although soft theorem is a relation between quantum scattering amplitudes - amplitudes with soft photon or graviton to amplitudes without soft photon or graviton - one can also relate soft theorem to classical scattering amplitudes. In four space-time dimensions this can be done via asymptotic symmetries [16, 31,34]. Ref. [88] produced a more direct relation between soft theorem and classical scattering in generic space-time dimensions by directly taking the classical limit of a quantum scattering amplitude. This relates various 
terms in soft theorem to appropriate terms in the radiative part of the electromagnetic and gravitational fields in classical scattering in generic space-time dimensions. Reversing the logic, one can use the classical scattering data to give an alternative definition of the soft factors.

Since classical scattering is well defined even in four space-time dimensions, one can hope to use the classical definition of soft factors to understand soft theorem in four dimensions. Since in higher dimensions the soft theorem expresses the low frequency radiative part of the electromagnetic and gravitational fields in terms of momenta and angular momenta of incoming and outgoing finite energy particles, the naive guess will be that the same formula will continue to hold in four dimensions. However in carrying out this procedure we encounter an obstacle $[89,90]$. The subleading terms in the soft theorem contain a factor of angular momentum $j^{\mu \nu}$ of the individual particles involved in the scattering, with the orbital contribution to the angular momentum given by $x^{\mu} p^{\nu}-x^{\nu} p^{\mu}$, where $x^{\mu}(\tau)$ and $p^{\mu}(\tau)$ label the asymptotic coordinates and momenta of the particle as a function of the proper time. In dimensions larger than four, $p^{\mu}$ approaches a constant and $x^{\mu}$ approaches the form $c^{\mu}+\alpha p^{\mu} \tau$ with constant $c^{\mu}$ and $\alpha$ as $\tau \rightarrow \infty$. Therefore $j^{\mu \nu}$ is independent of $\tau$ as $\tau \rightarrow \infty$ and we can use the asymptotic value of $j^{\mu \nu}$ computed this way to evaluate the soft factor. However in four space-time dimensions the long range gravitational and/or electromagnetic forces acting on the particles produce an additional term of the form $b^{\mu} \ln \tau$ in the expression for $x^{\mu}$. This gives a logarithmically divergent term of the form $\left(b^{\mu} p^{\nu}-b^{\nu} p^{\mu}\right) \ln \tau$ in the expression for $j^{\mu \nu}$, making the subleading soft factor divergent.

Since we do not expect the radiative component of the metric or gauge fields to diverge in classical scattering in four space-time dimensions, this suggests that the divergence in the subleading soft factor is a breakdown of the power series expansion in the energy $\omega$ of the soft particle. Therefore the soft factor must contain non-analytic terms in $\omega$. The natural guess is that the soft factor at the subleading order is given by replacing the factors of $\ln \tau$ in the naive expression by $\ln \omega^{-1}$. This has been tested in [89] by considering several examples of classical scattering in four space-time dimensions. ${ }^{1}$

The purpose of this paper is two fold. In all the examples considered in [89] the scattering process considered had one heavy center producing the long range Coulomb or gravitational field, and other particles carrying smaller masses were taken to be moving under the influence of the long range fields produced by the heavy center. In this paper we relax this assumption and consider a general scattering process where all particles involved in the scattering have masses of the same order, and then determine the logarithmic terms in the classical soft factor using the $\ln \tau \rightarrow \ln \omega^{-1}$ replacement rule. We also analyze directly the quantum subleading soft factor by considering one loop scattering of charged scalar fields in the presence of gravitational and electromagnetic interaction. The difference with the previous analysis, e.g. in [41], is that we do not insist on a power series expansion in $\omega$ and calculating the coefficients of the power series expansion. Instead we allow for possible nonanalytic terms of order $\ln \omega^{-1}$ in the soft expansion. This analysis yields results consistent

\footnotetext{
${ }^{1}$ The existence of various logarithmic terms in classical scattering has been known earlier [91-94]. Soft theorem provides a systematic procedure for computing the coefficient of the logarithmic term in the subleading soft factor without detailed knowledge of the forces responsible for the scattering.
} 
with the classical results, although the quantum results contain additional real part which we interpret as the result of back reaction of the radiation on the motion of the particles.

Since $[81,83,84]$ gave a general derivation of soft theorem including loop corrections as long as 1PI vertices do not generate soft factor in the denominator, one could ask to what extent we could derive the results of the current paper using the result of $[81,83,84]$. To this end we note that there are two distinct sources of logarithmic terms in the soft theorem. The first is the region of integration in which the loop momentum is large compared to the energy of the external soft particle. In this region we expect the arguments of $[81,83,84]$ to be valid, and we find that the contribution from this region can indeed be obtained by applying the usual soft operator on the amplitude without the soft graviton. The other source is the region of integration in which the loop momentum is small compared to the external soft momentum. The contribution from this region cannot be derived using the usual soft theorem, and need to be computed explicitly.

The rest of the paper is organized as follows. Section 2 contains a summary and a discussion of our results where we also discuss various special cases of our classical result. Section 3 describes the analysis of the logarithmic terms in the soft expansion for general classical scattering. Section 4 describes some general strategy for dealing with the infrared divergent part of the S-matrix and extracting the quantum soft factor by making use of momentum conservation. Section 5 describes one loop quantum computation of the logarithmic terms in the soft photon theorem in scalar quantum electrodynamics (scaler QED). Section 6 describes a similar computation in the soft graviton theorem in a theory of charge neutral scalars interacting with the gravitational field. In section 7 we consider charged scalars interacting via both gravitational and electromagnetic interaction, and determine the one loop contribution to the quantum soft graviton factor due to electromagnetic interaction and one loop contribution to the quantum soft photon factor due to gravitational interaction.

Classical gravitational radiation during a high energy scattering process has been analyzed in [95, 96]. We have been informed by G. Veneziano that for massless particle scattering, results related to the ones described here were found in [97], and also that the logarithmic terms in the classical scattering have been derived in [98] by taking the soft limit of the results of $[95,96]$.

Note added. The papers quoted above have now appeared in the arXiv [97, 98]. In particular the results of [98] can be shown to be in perfect agreement with our results for scattering of massless particles.

\section{Summary and analysis of the results}

In this section we shall first summarize our results and then discuss various aspects of the results. Finally we shall consider some special limits and compare with known results. We shall use $\hbar=c=8 \pi G=1$ units. 


\subsection{Summary of the results}

In order to give a uniform treatment of the classical soft photon and soft graviton theorem, we shall denote by $\phi(\vec{x}, t)$ the radiative part of the metric or electromagnetic field at a point $\vec{x}$ at time $t$ for a scattering event around the origin. For electromagnetic field, $\phi$ can be directly identified with the gauge field. For the gravitational field we define

$$
h_{\mu \nu}=\left(g_{\mu \nu}-\eta_{\mu \nu}\right) / 2, \quad e_{\mu \nu}=h_{\mu \nu}-\frac{1}{2} \eta_{\mu \nu} h_{\rho}^{\rho},
$$

and take $\phi$ to be $e_{\mu \nu}$. For both electromagnetism and gravity we define classical soft factor $S(\varepsilon, k)$ in $D$ space-time dimensions via the relation:

$$
\begin{aligned}
\int d t e^{i \omega t} \varepsilon \cdot \phi(\vec{x}, t) & =e^{i \omega R}\left(\frac{\omega}{2 \pi i R}\right)^{(D-2) / 2} \frac{1}{2 \omega} S(\varepsilon, k) \\
& =-\frac{i}{4 \pi R} e^{i \omega R} S(\varepsilon, k) \text { for } D=4,
\end{aligned}
$$

where $\varepsilon$ is the polarization tensor of the soft particle so that $\varepsilon \cdot \phi=\varepsilon^{\mu} A_{\mu}$ for gauge fields and $\varepsilon^{\mu \nu} e_{\mu \nu}$ for gravity, and

$$
k=-\omega(1, \hat{n}), \quad \hat{n} \equiv \vec{x} /|\vec{x}|, \quad R=|\vec{x}| .
$$

On the other hand the quantum soft factor $S(\varepsilon, k)$ is the ratio of an amplitude with an outgoing soft photon or graviton with momentum $k$ and polarization $\varepsilon$ and an amplitude without such a soft particle. It was shown in [88] that in the classical limit the quantum soft factor reduces to the classical soft factor for $D>4$. Our interest will be in analyzing the situation in $D=4$.

We consider the scattering of $n$ particles carrying electric charges $\left\{q_{a}\right\}$ and momenta $\left\{p_{a}\right\}$ for $a=1, \cdots n$. In our convention the momenta/charges carry extra minus sign if they are outgoing. The particles are taken to interact via electromagnetic and gravitational interactions besides other short range interactions whose nature we need not know. The symbol $\eta_{a}$ takes value $+1(-1)$ if the $a$-th particle is ingoing (outgoing). Then the classical result for the soft photon factor $S_{\mathrm{em}}(\varepsilon, k)$, containing terms of order $\omega^{-1}$ and $\ln \omega^{-1}$, is ${ }^{2}$

$$
\begin{aligned}
S_{\mathrm{em}}= & \sum_{a} \frac{\varepsilon_{\mu} p_{a}^{\mu}}{p_{a} \cdot k} q_{a}-i \ln \omega^{-1} \sum_{a} \frac{q_{a} \varepsilon_{\mu} k_{\rho}}{p_{a} \cdot k} \sum_{\substack{b \neq a \\
\eta_{a} \eta_{b}=1}} \frac{q_{a} q_{b}}{4 \pi} \frac{m_{a}^{2} m_{b}^{2}\left\{p_{b}^{\rho} p_{a}^{\mu}-p_{b}^{\mu} p_{a}^{\rho}\right\}}{\left\{\left(p_{b} \cdot p_{a}\right)^{2}-m_{a}^{2} m_{b}^{2}\right\}^{3 / 2}} \\
& +\frac{i}{4 \pi}\left(\ln \omega^{-1}+\ln R^{-1}\right) \sum_{\substack{b \\
\eta_{b}=-1}} k \cdot p_{b} \sum_{a} \frac{\varepsilon_{\mu} p_{a}^{\mu}}{p_{a} \cdot k} q_{a} \\
& +\frac{i}{8 \pi} \ln \omega^{-1} \sum_{a} \frac{q_{a} \varepsilon_{\mu} k_{\rho}}{p_{a} \cdot k} \sum_{\substack{b \neq a \\
\eta_{a} \eta_{b}=1}} \frac{p_{b} \cdot p_{a}}{\left\{\left(p_{b} \cdot p_{a}\right)^{2}-m_{a}^{2} m_{b}^{2}\right\}^{3 / 2}}\left(p_{b}^{\rho} p_{a}^{\mu}-p_{b}^{\mu} p_{a}^{\rho}\right)\left\{2\left(p_{b} \cdot p_{a}\right)^{2}-3 m_{a}^{2} m_{b}^{2}\right\} .
\end{aligned}
$$

\footnotetext{
${ }^{2}$ In this and subsequent expressions $R$ arises as an infrared cut-off. For the classical result the $\ln R$ terms arise due to long range gravitational force on the soft photon or graviton during its journey from the scattering center to the detector over a distance $R$. For the quantum part, the natural infrared cut-off is provided by the resolution of the detector. For a detector placed at a distance $R$ from the scattering center, the best energy resolution possible is of order $1 / R$. Therefore it is again natural to take $R$ as the infrared regulator.
} 
Since for real polarization the subleading contribution is purely imaginary, it does not affect the flux to this order. However the flux for circular polarization and/or the wave-form of the electromagnetic field do receive subleading contribution. An identical situation prevails for gravity.

The quantum result for $S_{\mathrm{em}}$ has additional terms: ${ }^{3}$

$$
\begin{aligned}
\Delta S_{\mathrm{em}}= & \frac{1}{16 \pi^{2}} \ln \omega^{-1} \sum_{a} q_{a} \frac{\varepsilon_{\mu} k_{\nu}}{p_{a} \cdot k}\left\{p_{a}^{\mu} \frac{\partial}{\partial p_{a \nu}}-p_{a}^{\nu} \frac{\partial}{\partial p_{a \mu}}\right\} \\
& \sum_{b \neq a}\left[\frac{\left\{2 q_{a} q_{b} p_{a} \cdot p_{b}+2\left(p_{a} \cdot p_{b}\right)^{2}-p_{a}^{2} p_{b}^{2}\right\}}{\sqrt{\left(p_{a} \cdot p_{b}\right)^{2}-p_{a}^{2} p_{b}^{2}}} \ln \left(\frac{p_{a} \cdot p_{b}+\sqrt{\left(p_{a} \cdot p_{b}\right)^{2}-p_{a}^{2} p_{b}^{2}}}{p_{a} \cdot p_{b}-\sqrt{\left(p_{a} \cdot p_{b}\right)^{2}-p_{a}^{2} p_{b}^{2}}}\right)\right] \\
& -\frac{1}{8 \pi^{2}}\left(\ln \omega^{-1}+\ln R^{-1}\right) \sum_{a} \frac{q_{a} \varepsilon_{\mu} p_{a}^{\mu}}{p_{a} \cdot k} \sum_{b}\left(p_{b} \cdot k\right) \ln \left(\frac{m_{b}^{2}}{\left(p_{b} \cdot \hat{k}\right)^{2}}\right) .
\end{aligned}
$$

The classical results are universal, independent of the theory and the nature of external particles. We expect that the quantum results are also universal, but we have derived them by working with one loop amplitudes in scalar QED coupled to gravity. It is easy to check that (2.4), (2.5) are invariant under gauge transformation $\varepsilon_{\mu} \rightarrow \varepsilon_{\mu}+\xi k_{\mu}$ for any constant $\xi$.

As will be discussed in section 2.2, the quantum correction (2.5) should not be directly added to (2.4) and substituted into (2.2) to compute the radiative component of the classical electromagnetic field. Rather, when the contribution (2.5) is small compared to (2.4), we can substitute (2.4) into (2.2) to compute the classical electromagnetic field produced by a scattering event.

As discussed in section 4, the quantum results are ambiguous and are defined up to addition of a term to $S_{\mathrm{em}}$ of the form $\ln R^{-1} k \cdot U S_{\mathrm{em}}^{(0)}$ where $S_{\mathrm{em}}^{(0)}$ is the leading soft factor given by the first term on the right hand side of (2.4) and $U$ is a vector constructed out of the $p_{a}$ 's. By choosing $U=\left(8 \pi^{2}\right)^{-1} \sum_{b} p_{b} \ln \left(m_{b}^{2} / \mu^{2}\right)$, we can replace the $\ln m_{b}^{2}$ term in the coefficient of $\ln R^{-1}$ in the last line of (2.5) by $\ln \mu^{2}$ for any mass parameter $\mu$. This makes manifest the fact that the coefficient is not divergent in the $m_{b} \rightarrow 0$ limit. The coefficient of $\ln \omega^{-1}$ cannot be changed this way, but in this case the finiteness of $m_{b} \rightarrow 0$ limit follows as a consequence of cancellation between the second and third line of (2.5) and momentum conservation.

If we want to consider the situation where we ignore the effect of gravity, then we need to set the terms proportional to $\ln \omega^{-1}$ that are linear in $q_{c}$ 's to zero. On the other hand if we want to consider the situation where we ignore the effect of electromagnetic interaction between the particles during scattering (but still use electromagnetic interaction to compute soft photon emission process), we have to set the terms proportional to $\ln \omega^{-1}$ that are cubic in the $q_{c}$ 's to zero.

\footnotetext{
${ }^{3}$ Note however that when we express the results in terms of the frequency/wavelength of the soft photon/graviton and momenta of the finite energy particles, neither the classical nor the quantum result has any power of $\hbar$. We shall discuss later the conditions under which we expect the quantum results to be small compared to the classical results.
} 
The classical result for soft graviton factor takes the form

$$
\begin{aligned}
S_{\mathrm{gr}}= & \sum_{a} \frac{\varepsilon_{\mu \nu} p_{a}^{\mu} p_{a}^{\nu}}{p_{a} \cdot k}-i \ln \omega^{-1} \sum_{a} \frac{\varepsilon_{\mu \nu} p_{a}^{\nu} k_{\rho}}{p_{a} \cdot k} \sum_{\substack{b \neq a \\
\eta_{a} \eta_{b}=1}} \frac{q_{a} q_{b}}{4 \pi} \frac{m_{a}^{2} m_{b}^{2}\left\{p_{b}^{\rho} p_{a}^{\mu}-p_{b}^{\mu} p_{a}^{\rho}\right\}}{\left\{\left(p_{b} \cdot p_{a}\right)^{2}-m_{a}^{2} m_{b}^{2}\right\}^{3 / 2}} \\
& +\frac{i}{4 \pi}\left(\ln \omega^{-1}+\ln R^{-1}\right) \sum_{\substack{b \\
\eta_{b}=-1}} k \cdot p_{b} \sum_{a} \frac{\varepsilon_{\mu \nu} p_{a}^{\mu} p_{a}^{\nu}}{p_{a} \cdot k} \\
& +\frac{i}{8 \pi} \ln \omega^{-1} \sum_{a} \frac{\varepsilon_{\mu \nu} p_{a}^{\nu} k_{\rho}}{p_{a} \cdot k} \sum_{\substack{b \neq a \\
\eta_{a} \eta_{b}=1}} \frac{p_{b} \cdot p_{a}}{\left\{\left(p_{b} \cdot p_{a}\right)^{2}-m_{a}^{2} m_{b}^{2}\right\}^{3 / 2}}\left(p_{b}^{\rho} p_{a}^{\mu}-p_{b}^{\mu} p_{a}^{\rho}\right)\left\{2\left(p_{b} \cdot p_{a}\right)^{2}-3 m_{a}^{2} m_{b}^{2}\right\} .
\end{aligned}
$$

The quantum result has additional terms

$$
\begin{aligned}
\Delta S_{\mathrm{gr}}= & \frac{1}{16 \pi^{2}} \ln \omega^{-1} \sum_{a} \frac{\varepsilon_{\mu \rho} p_{a}^{\rho} k_{\nu}}{p_{a} \cdot k}\left\{p_{a}^{\mu} \frac{\partial}{\partial p_{a \nu}}-p_{a}^{\nu} \frac{\partial}{\partial p_{a \mu}}\right\} \\
& \sum_{b \neq a}\left[\frac{\left\{2 q_{a} q_{b} p_{a} \cdot p_{b}+2\left(p_{a} \cdot p_{b}\right)^{2}-p_{a}^{2} p_{b}^{2}\right\}}{\sqrt{\left(p_{a} \cdot p_{b}\right)^{2}-p_{a}^{2} p_{b}^{2}}} \ln \left(\frac{p_{a} \cdot p_{b}+\sqrt{\left(p_{a} \cdot p_{b}\right)^{2}-p_{a}^{2} p_{b}^{2}}}{p_{a} \cdot p_{b}-\sqrt{\left(p_{a} \cdot p_{b}\right)^{2}-p_{a}^{2} p_{b}^{2}}}\right)\right] \\
& -\frac{1}{8 \pi^{2}}\left(\ln \omega^{-1}+\ln R^{-1}\right) \sum_{a} \frac{\varepsilon_{\mu \nu} p_{a}^{\mu} p_{a}^{\nu}}{p_{a} \cdot k} \sum_{b} p_{b} \cdot k \ln \frac{m_{b}^{2}}{\left(p_{b} \cdot \hat{k}\right)^{2}}
\end{aligned}
$$

where $\hat{k}=-k / \omega=(1, \hat{n})$. Again the classical results are valid universally. The quantum results are obtained from one loop calculation in scalar QED coupled to gravity, but we expect them to be universal. As in the case of (2.5), the $\ln m_{b}^{2}$ term in the coefficient of $\ln R^{-1}$ in the last line of (2.7) can be replaced by $\ln \mu^{2}$ by exploiting the ambiguity in the definition of the soft factor discussed in section 4. One can check that (2.6), (2.7) are invariant under gauge transformations $\varepsilon_{\mu \nu} \rightarrow \varepsilon_{\mu \nu}+\xi_{\mu} k_{\nu}+\xi_{\nu} k_{\mu}$ for any constant vector $\xi_{\mu}$.

If we want to consider the situation where we ignore the effect of electromagnetic interactions, then we need to set the terms proportional to $\ln \omega^{-1}$ that are quadratic in $q_{c}$ 's to zero. On the other hand if we want to consider the situation where we ignore the effect of gravitational interaction between the particles during scattering (but still use gravitational interaction to compute soft graviton emission process), we have to set the $q_{c}$ independent terms in the coefficient of $\ln \omega^{-1}$ to zero.

\subsection{Discussion of results}

First we shall briefly outline how these results are derived; more details can be found in later sections. The classical results (2.4) and (2.6) are the result of direct application of classical soft theorem to subleading order. As described in [89], the soft factor involves orbital angular momenta of initial and final particles and these diverge logarithmically in the elapsed time $\tau$ in four dimensions due to the long range gravitational/electromagnetic force on the incoming and outgoing particles that generates a term proportional to $\ln |\tau|$ in the trajectory. We follow the prescription of [89] of replacing $\ln |\tau|$ by $\ln \omega^{-1}$ to arrive at the first and third lines of the classical results (2.4), (2.6). The second lines of (2.4) 
and (2.6) arise from additional phases that are not directly determined by soft theorem. They represent the effect of long range gravitational force on the outgoing soft photon or graviton which causes the soft particle to slow down and also backscatter.

Quantum results are the result of direct one loop computation in a field theory of multiple charged scalars, coupled to electromagnetic and gravitational fields. We simply evaluate the order $\omega^{-1}$ and $\ln \omega^{-1}$ terms in the scattering amplitude of multiple finite energy scalars and an outgoing soft photon or graviton of energy $\omega$, and express this as the product of the amplitude without the soft photon or graviton and a multiplicative factor that we call the soft factor. The latter is given by the sum of (2.4) and (2.5) for soft photon and the sum of (2.6) and (2.7) for the soft graviton. Even though the S-matrix elements with and without the soft particle are infrared divergent, much of this cancels when we take the ratio of the two. The remaining infrared divergent part is regulated by the infra-red length cut-off $R$ and is responsible for the terms proportional to $\ln R$ in these expressions. This is related to the quantity $\sigma_{n}^{\prime}$ introduced in [41].

The different terms proportional to $\ln \omega^{-1}$ in $(2.4),(2.5)$ and in (2.6), (2.7) have different origin. We shall explain them in the context of the soft graviton factor, but the case of soft photon factor is very similar.

1. We begin with the classical result (2.6). The term proportional to $q_{a} q_{b}$ in the first line represents the effect of late time gravitational radiation due to the late time acceleration of the particles via long range electromagnetic interaction. The term in the last line of (2.6) represents the effect of late time gravitational radiation due to the late time acceleration of the particles via long range gravitational interaction. We expect the scale of these logarithms to be set by the largest length scale involved in the classical scattering process, e.g. the typical distance of closest approach between the particles involved in the scattering. This is taken to be larger than or of the order of the Schwarzschild radii of the particles and much larger than the Compton wave-lengths of the particles involved in the scattering. In the quantum one loop computation both these terms arise from the region of loop momentum integration where the loop momentum is large compared to $\omega$ but small compared to the energies of the other particles. In this case the scale of these logarithms is again set by the largest length scale involved in the quantum scattering which is the inverse of the typical energy carried by the finite energy external states. For one loop result to be reliable, this needs to be taken to be large compared to the Schwarzschild radii of these particles.

2. The term in the second line of (2.6) proportional to $\left(\ln \omega^{-1}+\ln R^{-1}\right)$ represents the effect of gravitational drag on the soft graviton due to the other finite energy particles in the final state. This has the effect of causing a time delay, represented by the $\ln R^{-1}$ term, for the soft graviton to travel to a distance $R$. This also has the effect of inducing backscattering of the soft graviton, represented by the $\ln \omega^{-1}$ term. In the quantum computation these terms arise from region of loop momentum integration where the loop momentum is smaller than $\omega$ and larger than the infrared cut-off $R^{-1}$. This term has appeared e.g. in [91, 93, 94]. As mentioned in footnote 2, 
the scale of these logarithms is set by the effective infrared cut-off, e.g. the distance $R$ to the detector for the classical scattering and the resolution of the detector for the quantum scattering. The latter in turn has a lower limit set by $R^{-1}$ since we cannot measure the energy of the outgoing particle with an accuracy better than $R^{-1}$ if the detector is placed at a distance $R$ from the scattering center.

3. We emphasize that the classical results are obtained by replacing in the classical soft theorem the logarithmically divergent terms by $\ln \omega^{-1}$ and not by direct calculation of electromagnetic and gravitational radiation during classical scattering. In special cases the equivalence of these two procedures was tested in [89] by direct classical computation. In principle similar tests can be done for the general formulae (2.4) and (2.6), but we have not done this.

4. We now turn to the additional terms (2.7) that arise in the quantum computation. First note that both these terms are real for real polarizations unlike the classical result where the coefficients of $\ln \omega^{-1}$ terms are imaginary for real polarizations. The terms in the first two lines come from regions of loop momentum integration where the loop momentum is large compared to $\omega$ but small compared to the energies of the other particles, while the term in the third line arise from region of loop momentum integration where the loop momentum is small compared to $\omega$ and large compared to the infrared cut-off $R^{-1}$.

5. In the quantum computation the terms that arise from loop momenta large compared to $\omega$, namely the terms in the first and third line of (2.6) and the first two lines of (2.7), can be generated using a simple algorithm. As discussed earlier, the amplitude without the soft graviton has an infrared divergent factor multiplying it. Let us call this the IR factor. If in the integration over loop momenta of this IR factor we restrict the loop momentum integration to be large compared to $\omega$ and apply the usual subleading soft differential operator that arises in higher dimensions to this IR factor, we recover precisely the results given in the first and third line of (2.6) and the first two lines of (2.7). The rest of the contribution that arises from integration region where the loop momentum is small compared to $\omega$ cannot be recovered this way. This indicates that the general argument of $[81,83]$, based on general coordinate invariance of 1PI effective action and power counting assuming that loops do not generate inverse power of soft momentum, remain valid in four dimensions as well as long as the loop momentum is large compared to the external soft momentum.

Since the real infrared divergent part of the amplitude reflects the effect of real graviton emission, our interpretation of the extra contributions (2.7) in the quantum theory is that they reflect the effect of backreaction of soft radiation on the classical trajectories. To this end note that the validity of the classical limit described in [88] requires that the total energy carried by soft radiation should remain small compared to the energies of the finite energy objects taking part in the scattering. Here 'soft radiation' represents those particles which are not included in the sum over $a$ in (2.6). Therefore we should expect that the extra terms arising in the quantum theory should be small in the limit when the total energy carried by the soft radiation is small. 
In order to test this hypothesis we need to consider a scattering where the energy carried away by soft radiation remains small compared to the energies of finite energy objects. One way to achieve this is to consider scattering at large impact parameter so that each incoming particle gets deflected by a small amount and the energy radiated during this process remains small. In this case the momenta $\left\{p_{a}\right\}$ come in approximately equal and opposite pairs - the incoming and the corresponding outgoing particle. Now in eq. (2.7) the last term changes sign under $p_{b} \rightarrow-p_{b}$ and also under $p_{a} \rightarrow-p_{a}$. This shows that it is small for small deflection scattering. The first term on the right hand side of (2.7) changes sign under $\left(p_{b}, q_{b}\right) \rightarrow-\left(p_{b}, q_{b}\right)$ and also under $\left(p_{a}, q_{a}\right) \rightarrow-\left(p_{a}, q_{a}\right)$, due to the argument of the log getting inverted under each of these operations. This shows that the terms approximately cancel making the result small. There is one exception to this that arises when $q_{b}=-q_{a}, p_{b} \simeq-p_{a}$, i.e. the pairs $(a, b)$ represent the incoming and the corresponding outgoing particle. In this case there is no other term that cancels this since the sum does not include the $b=a$ term, and we need to explicitly evaluate this and show that it vanishes. This can be checked explicitly by first evaluating the derivatives in the second line of (2.7), then setting $p_{b}=-p_{a}+\epsilon$ and then carefully evaluating the result in the $\epsilon \rightarrow 0$ limit. Even though individual terms diverge in the $\epsilon \rightarrow 0$ limit, a careful analysis shows that the result vanishes. This confirms that quantum corrections are small in this limit.

Another situation discussed in [88], where the radiated energy remains small compared to the energies of the hard particles, is the probe limit in which one of the particles has a large mass $M$ and the other particles are lighter carrying energy small compared to $M$. We shall now verify that in this case too the quantum corrections (2.7) are small compared to the classical result (2.6). For this we shall work in a frame in which the heavy particle is initially at rest, and using gauge invariance choose the polarization tensor $\varepsilon$ to have only spatial components. After the scattering the heavy particle acquires a momentum but it is small compared to $M$. In this case the dominant contribution to (2.6), of order $M$, comes from choosing $a$ to be one of the light particles and $b$ to be the heavy particle in the second and third line of (2.6). However in the quantum correction (2.7) similar contribution cancels between the choice of $b$ as the initial state heavy particle and the final state heavy particle, and we do not get any contribution proportional to $M$. This again shows that quantum corrections are small compared to the classical result in this limit.

We must emphasize however that the quantum analysis is carried out for single soft graviton emission. If we want to relate the quantum result to the radiative component of the classical gravitational field as in [88], then we need to first consider multiple soft graviton emission and then take the classical limit. The analysis of [88] relied on the fact that the soft factors associated with different bins in the phase space are independent of each other, i.e. the probability of emitting certain number of soft particles in one bin does not depend on how many soft particles are emitted in the other bin. This independence breaks down when the total energy carried by the soft particles becomes comparable to the energies of the hard particles - precisely when the quantum correction (2.7) becomes comparable to the classical result (2.6). Therefore we should not use (2.7) to modify the classical result (2.6). Instead we should use the smallness of (2.7) as a test of when the classical result (2.6) is valid. An identical discussion holds for electromagnetism. 


\subsection{Special cases}

As a special case we can consider the situation described in [90] where a neutral massive object of mass $M$ at rest decays into a heavy object of mass $M_{0} \simeq M$ and a set of neutral light objects carrying mass $m_{a} \ll M$ and momentum $p_{a}=-e_{a}\left(1, \vec{\beta}_{a}\right)$ with $e_{a} \ll M$ for $a=1, \cdots N$. Our goal will be to write down the classical soft graviton factor for this case. We shall take the polarization tensor of the soft graviton to have components only along the spatial direction, since the result for the other components may be found by using invariance under the gauge transformation $\varepsilon_{\mu \nu} \rightarrow \varepsilon_{\mu \nu}+\xi_{\mu} k_{\nu}+\xi_{\nu} k_{\mu}$ for any vector $\xi$. If we denote the momentum carried by final state heavy object of mass $M_{0}$ by $p_{N+1}$, then we have $p_{N+1}^{0} \simeq-M_{0}$ and $\left|p_{N+1}^{i}\right| \ll M_{0}$. Examining (2.6) with $q_{a}=q_{b}=0$ we see that dominant term proportional to $\ln \omega^{-1}$ comes from the terms where we choose $b=N+1$ and $a$ labels any of the $N$ finite energy states. Using the relation $e_{a}^{2}=m_{a}^{2} /\left(1-\vec{\beta}_{a}^{2}\right)$, the net contribution takes the form:

$$
\begin{aligned}
& \frac{i}{4 \pi} \ln \omega^{-1} M_{0} \sum_{a=1}^{N} e_{a} \frac{\varepsilon^{i j} \beta_{a i} \beta_{a j}}{1-\hat{n} \cdot \vec{\beta}_{a}}+\frac{i}{8 \pi} \ln \omega^{-1} M_{0} \sum_{a=1}^{N} e_{a} \frac{\varepsilon^{i j} \beta_{a i} \beta_{a j}}{1-\hat{n} \cdot \vec{\beta}_{a}} \frac{\left(-e_{a}\right)\left(2 e_{a}^{2}-3 m_{a}^{2}\right)}{\left(e_{a}^{2}-m_{a}^{2}\right)^{3 / 2}} \\
& =\frac{i}{8 \pi} \ln \omega^{-1} M_{0} \sum_{a=1}^{N} e_{a} \frac{\varepsilon^{i j} \beta_{a i} \beta_{a j}}{1-\hat{n} \cdot \vec{\beta}_{a}} \frac{2 \vec{\beta}_{a}^{3}+1-3 \vec{\beta}_{a}^{2}}{\left|\vec{\beta}_{a}\right|^{3}}+\cdots,
\end{aligned}
$$

where $\cdots$ contain terms without a factor of $M_{0}$ and are therefore smaller in the limit of large $M_{0}$. This agrees with the results of [90]. As discussed in [90], this produces a late time tail in the gravitational wave-form that falls off as inverse power of time.

Note that when all the final state light particles are massless, so that $\left|\vec{\beta}_{a}\right|=1$ for $1 \leq a \leq N$, the expression (2.8) vanishes. This would be the situation during binary black hole merger when the final state particles are only gravitons. However since in such processes the radiation carries away an appreciable fraction of the mass of the parent system, the $\cdots$ terms in (2.8) could be significant even though their contribution will be suppressed by the ratio of the total energy carried away by radiation to the mass of the parent system. We shall now evaluate the result without making any approximation. In this case in the sum over $a$ and $b$ in (2.6), either $a$ or $b$ (or both) represents a massless particle. Recalling that when $p_{a}$ and $p_{b}$ are both outgoing then $p_{a} \cdot p_{b}$ is negative, we can express the terms in (2.6) proportional to $\ln \omega^{-1}$ as

$$
\frac{i}{4 \pi} \ln \omega^{-1} \sum_{a=1}^{N+1} \varepsilon^{i j} p_{a i} p_{a j}+\frac{i}{4 \pi} \ln \omega^{-1} \sum_{a=1}^{N+1} \varepsilon^{i j} p_{a i} \sum_{\substack{b=1 \\ b \neq a}}^{N+1} p_{b j}=0,
$$

where in the last step we have used conservation of spatial momentum $\sum_{b=1}^{N+1} p_{b j}=0$. Therefore we see that even without making any approximation, the coefficient of the $\ln \omega^{-1}$ term in the classical soft graviton factor continues to vanish.

Another special case we can consider is when a charge neutral object of mass $M$ at rest breaks apart into two charge neutral objects of masses $m_{1}$ and $m_{2}$, spatial momenta $\vec{p}$ and $-\vec{p}$ and energies $e_{1}=\sqrt{m_{1}^{2}+\vec{p}^{2}}$ and $e_{2}=\sqrt{\vec{p}^{2}+m_{2}^{2}}$. In this case if we take the polarization tensor of the soft graviton to have components only along the spatial direction, 
then the contribution from the initial state to (2.6) vanishes and we need to only compute the contribution from a pair of final states. This can be easily evaluated and the terms proportional to $\ln \omega^{-1}$ take the form

$$
\begin{aligned}
\frac{i}{8 \pi} \ln \omega^{-1} & \varepsilon_{i j} p^{i} p^{j}\left(e_{1}+e_{2}\right)\left\{\frac{1}{e_{1}-\hat{n} \cdot \vec{p}}+\frac{1}{e_{2}+\hat{n} \cdot \vec{p}}\right\} \\
\times & {\left[\frac{e_{1} e_{2}+\vec{p}^{2}}{\left\{\left(e_{1} e_{2}+\vec{p}^{2}\right)^{2}-m_{1}^{2} m_{2}^{2}\right\}^{3 / 2}}\left\{2\left(e_{1} e_{2}+\vec{p}^{2}\right)^{2}-3 m_{1}^{2} m_{2}^{2}\right\}-2\right] . }
\end{aligned}
$$

Next special case we shall analyze is that of scattering of massless particles, again focussing on the classical result (2.6). Defining

$$
P \equiv \sum_{\eta_{a}=1} p_{a}=-\sum_{\eta_{a}=-1} p_{a}
$$

and the fact that $p_{a} \cdot p_{b}$ is negative for $\eta_{a} \eta_{b}=1$, we can express the term proportional to $\ln \omega^{-1}$ in (2.6) for massless particles as

$$
-\frac{i}{2 \pi} \ln \omega^{-1} k . P \sum_{\substack{a, b \\ b \neq a, \eta_{a}=\eta_{b}=1}} \frac{\varepsilon_{\mu \nu} p_{a}^{\mu} p_{a}^{\nu}}{p_{a} \cdot k}+\frac{i}{2 \pi} \ln \omega^{-1} \varepsilon_{\mu \nu} P^{\mu} P^{\nu} .
$$

Note that this involves only the momenta of the initial state particles and is insensitive to the momenta of the final state particles. This asymmetry is related to the fact that in our analysis we are considering soft particle only in the final state and not in the initial state.

More generally one can show that for a general scattering process involving both massive and massless particles, the terms proportional to $\ln \omega^{-1}$ in the classical formula (2.6) is not sensitive to the details of the final state massless particles except through overall momentum conservation. To see this let us first consider terms that could involve a final state massless particle momenta and the initial state momenta. These come from choosing $a$ to be an initial state and $b$ to be a final state massless state in the term in the second line of (2.6). The net contribution from such terms is given by

$$
\frac{i}{4 \pi} \ln \omega^{-1} \sum_{\substack{b \text { massless } \\ \eta_{\mathrm{b}}=-1}} k \cdot p_{b} \sum_{\substack{a \\ \eta_{a}=1}} \frac{\varepsilon_{\mu \nu} p_{a}^{\mu} p_{a}^{\nu}}{k \cdot p_{a}}=-\frac{i}{4 \pi} \ln \omega^{-1} k \cdot\left(P-P_{\text {massive }}\right) \sum_{\substack{a \\ \eta_{a}=1}} \frac{\varepsilon_{\mu \nu} p_{a}^{\mu} p_{a}^{\nu}}{k \cdot p_{a}},
$$

where $-P$ denotes total outgoing momentum as defined in $(2.11)$ and $-P_{\text {massive }}$ denotes the total outgoing momentum carried by the massive particles. Therefore this does not depend explicitly on the momenta of the outgoing massless states except through momentum conservation.

Next we consider terms that involve a pair of final state momenta at least one of which is massless. This term receives contribution from all three lines on the right hand side of (2.6) with the restriction $\eta_{a}=1, \eta_{b}=1$, and either $m_{a}$ or $m_{b}$ or both zero. Therefore the term proportional to $q_{a} q_{b}$ vanishes. Also the coefficient of $\ln \omega^{-1}$ in the summand in the last two lines simplifies to

$$
\frac{i}{4 \pi} \frac{\varepsilon_{\mu \nu} p_{a}^{\mu} p_{a}^{\nu}}{p_{a} \cdot k} p_{b} \cdot k-\frac{i}{4 \pi} \frac{\varepsilon_{\mu \nu} p_{a}^{\mu} p_{a}^{\nu}}{p_{a} \cdot k} p_{b} \cdot k+\frac{i}{4 \pi} \varepsilon_{\mu \nu} p_{a}^{\mu} p_{b}^{\nu} .
$$


In the first term the sum over $a$ and $b$ includes the term where $b=a$, but in the second and the third term the sum excludes the $b=a$ term. Therefore the first two terms almost cancel, leaving behind a contribution where we set $b=a$. This left over contribution $\frac{i}{4 \pi} \varepsilon_{\mu \nu} p_{a}^{\mu} p_{a}^{\nu}$ can now be added to the last term to include in the sum over $a$ or $b$ also the contribution where $b=a$. The net contribution from the terms where either $a$ or $b$ or both represent massless state is then

$$
\frac{i}{4 \pi} \ln \omega^{-1} \sum_{\substack{a, b ; \eta_{a}=\eta_{b}=-1 \\ \text { either a or b massless }}} \varepsilon_{\mu \nu} p_{a}^{\mu} p_{b}^{\nu} .
$$

This can be rewritten as

$$
\frac{i}{4 \pi} \ln \omega^{-1} \varepsilon_{\mu \nu}\left(\sum_{a, b ; \eta_{a}=\eta_{b}=-1} p_{a}^{\mu} p_{b}^{\nu}-\sum_{\substack{a, b ; \eta_{a}=\eta_{b}=-1 \\ \text { and } \mathrm{b} \text { massive }}} p_{a}^{\mu} p_{b}^{\nu}\right)=\frac{i}{4 \pi} \ln \omega^{-1} \varepsilon_{\mu \nu}\left(P^{\mu} P^{\nu}-P_{\text {massive }}^{\mu} P_{\text {massive }}^{\nu}\right) .
$$

This also does not depend on the details of the momenta of massless final state particles except for the total momentum carried by these particles.

\section{Classical analysis}

The goal of this section will be to calculate the logarithmic terms in the soft factors in four space-time dimensions by examining them in the classical limit.

In dimensions larger than 4, the soft factors for photons and gravitons are given respectively by

$$
S_{\mathrm{em}}=\sum_{a} \frac{\varepsilon_{\mu} p_{a}^{\mu}}{p_{a} . k} q_{a}+i \sum_{a} q_{a} \frac{\varepsilon_{\mu} k_{\rho} \mathbf{J}_{a}^{\rho \mu}}{p_{a} \cdot k},
$$

and

$$
S_{\mathrm{gr}}=\sum_{a} \frac{\varepsilon_{\mu \nu} p_{a}^{\mu} p_{a}^{\nu}}{p_{a} . k}+i \sum_{a} \frac{\varepsilon_{\mu \nu} p_{a}^{\nu} k_{\rho} \mathbf{J}_{a}^{\rho \mu}}{p_{a} . k} .
$$

Here the sum over $a$ runs over all the incoming and outgoing particles, and $q_{a}, p_{a}$ and $\mathbf{J}_{a}$ denote the charge, momentum and angular momentum of the $a$-th particle, counted with positive sign for an ingoing particle and negative sign for an outgoing particle. $S_{\mathrm{em}}$ may also contain a non-universal term at the subleading order. For S-matrix elements in quantum theory, $\mathbf{J}_{a}$ is a differential operator involving derivatives with respect to the external momenta. However in the classical limit in which the external finite energy states are macroscopic, $\mathbf{J}_{a}$ represents the classical angular momenta carried by the external particles. In this limit the soft factors describe the radiative part of the low frequency electromagnetic and gravitational fields during a classical scattering [88] as described in (2.2).

In applying (3.1), (3.2) to four dimensional theories, the complication arises from the contribution to $\mathbf{J}_{a}^{\mu \nu}$ from the orbital angular momentum. They are computed from the form of the asymptotic trajectories:

$$
r_{a}^{\mu}(\sigma)=\eta_{a} \frac{1}{m_{a}} p_{a}^{\mu} \sigma+c_{a}^{\mu} \ln |\sigma|+\cdots
$$


where $\eta_{a}$ is positive for incoming particles and negative for outgoing particles, $m_{a}$ is the mass of the $a$-th particle and the proper time $\sigma$ is large and negative for incoming particles and large and positive for outgoing particles. The term proportional to $\ln |\sigma|$ represents the effect of long range electromagnetic and/or gravitational interaction between the particles. This gives, for large $|\sigma|$,

$$
\mathbf{J}_{a}^{\mu \nu} \simeq r_{a}^{\mu}(\sigma) p_{a}^{\nu}-r_{a}^{\nu}(\sigma) p_{a}^{\mu}+\operatorname{spin}=\left(c_{a}^{\mu} p_{a}^{\nu}-c_{a}^{\nu} p_{a}^{\mu}\right) \ln |\sigma|+\cdots .
$$

Here and in the following we shall use the convention that when a variable is followed by an argument $(\sigma)$ it denotes the value of the variable at proper time $\sigma$, but when a variable is written without an argument, we take it to be its $\sigma$ independent asymptotic value. Therefore in (3.3), (3.4) the $p_{a}^{\mu}$ 's denote the asymptotic values of $p_{a}^{\mu}$, reflecting the fact that the difference between $p_{a}^{\mu}(\sigma)=m_{a} \eta_{a} d r_{a}^{\mu} / d \sigma$ and $p_{a}^{\mu}$ approaches zero asymptotically.

Analysis of [89] indicates that if we substitute (3.4) into (3.1) and (3.2) and replace $\ln |\sigma|$ by $\ln \omega^{-1}$ - where $\omega=k_{0}$ is the frequency of the outgoing soft radiation - we can recover the logarithmic terms in the soft factors up to overall phases. This gives, up to overall phases:

$$
S_{\mathrm{em}}=\sum_{a} \frac{\varepsilon_{\mu} p_{a}^{\mu}}{p_{a} . k} q_{a}+i \ln \omega^{-1} \sum_{a} q_{a} \frac{\varepsilon_{\mu} k_{\rho}\left(c_{a}^{\rho} p_{a}^{\mu}-c_{a}^{\mu} p_{a}^{\rho}\right)}{p_{a} . k}
$$

and

$$
S_{\mathrm{gr}}=\sum_{a} \frac{\varepsilon_{\mu \nu} p_{a}^{\mu} p_{a}^{\nu}}{p_{a} \cdot k}+i \ln \omega^{-1} \sum_{a} \frac{\varepsilon_{\mu \nu} p_{a}^{\nu} k_{\rho}\left(c_{a}^{\rho} p_{a}^{\mu}-c_{a}^{\mu} p_{a}^{\rho}\right)}{p_{a} \cdot k} .
$$

Note that although $S_{\mathrm{em}}$ may contain a non-universal term at the subleading order, the term proportional to $\ln \omega^{-1}$ comes from orbital angular momentum and is universal.

Irrespective of what forces are operative during the scattering, the coefficient $c_{a}^{\mu}$ are determined only by the long range forces acting on the incoming and the outgoing particles. These will be taken to be electromagnetic and/or gravitational interaction. We shall now compute $c_{a}^{\mu}$ due to electromagnetic and gravitational interactions. We know from explicit comparison with known results that in the case of scattering via electromagnetic interactions there are no additional phases in the soft factor, but in the case of gravitational long range interaction there is an additional phase reflecting the effect of backscattering of the soft photon or soft graviton in the background gravitational field [91, 93, 94]. This phase will also be determined below.

\subsection{Effect of electromagnetic interactions}

We shall first study the effect of logarithmic correction to the trajectory due to long range electromagnetic interaction. For this we need to compute the gauge potential $A_{\mu}^{(b)}(x)$ at space-time point $x$ due to particle $b$. We have

$$
A_{\mu}^{(b)}(x)=\frac{1}{2 \pi} \int d \sigma \eta_{b} q_{b} V_{b \mu}(\sigma) \delta_{+}\left(-\left(x-r_{b}(\sigma)\right)^{2}\right), \quad V_{b}^{\mu}(\sigma) \equiv \frac{d r_{b \mu}(\sigma)}{d \sigma} \simeq \eta_{b} \frac{p_{b}^{\mu}}{m_{b}}
$$

where $\delta_{+}$denotes the usual Dirac delta function with the understanding that we have to choose the zero of the argument for which $x^{0}>r_{b}^{0}(\sigma)$. $V_{b}$ denotes the asymptotic four 
velocity of the $b$-th particle. In evaluating (3.7) we shall ignore the logarithmic corrections to the trajectory and take $r_{b}(\sigma) \simeq V_{b} \sigma$. This gives, using $V_{b}^{2}=-1$,

$\delta_{+}\left(-\left(x-r_{b}(\sigma)\right)^{2}\right)=\delta_{+}\left(-x^{2}+2 V_{b} \cdot x \sigma+\sigma^{2}+\cdots\right) \simeq \frac{1}{2\left|V_{b} \cdot x+\sigma\right|} \delta\left(\sigma+V_{b} \cdot x+\sqrt{\left(V_{b} \cdot x\right)^{2}+x^{2}}\right)$,

where the sign in front of the square root has been chosen to ensure that $x^{0}>x_{b}^{0}(\sigma)$ at the solution. Substituting this into (3.7) we get

$$
A_{\mu}^{(b)}(x) \simeq \frac{1}{4 \pi} \frac{\eta_{b} q_{b} V_{b \mu}}{\sqrt{\left(V_{b} \cdot x\right)^{2}+x^{2}}} .
$$

From this we calculate

$$
F_{\mu \nu}^{(b)}(x)=\partial_{\mu} A_{\nu}^{(b)}(x)-\partial_{\nu} A_{\mu}^{(b)}(x) \simeq-\frac{\eta_{b} q_{b}}{4 \pi} \frac{x_{\mu} V_{b \nu}-x_{\nu} V_{b \mu}}{\left\{\left(V_{b} \cdot x\right)^{2}+x^{2}\right\}^{3 / 2}} .
$$

At the location $r_{a}=V_{a} \sigma=-V_{a}|\sigma| \eta_{a}$ of the $a$-th particle we get, using $V_{a}^{2}=-1$

$$
F_{\mu \nu}^{(b)}\left(r_{a}(\sigma)\right) \simeq \eta_{a} \eta_{b} \frac{q_{b}}{4 \pi \sigma^{2}} \frac{V_{a \mu} V_{b \nu}-V_{a \nu} V_{b \mu}}{\left\{\left(V_{b} \cdot V_{a}\right)^{2}-1\right\}^{3 / 2}} .
$$

Now the $a$-th particle will feel the field produced by the $b$-th particle if either both $a$-th and the $b$-th particle are outgoing or if both particles are ingoing. Therefore the equation of motion for the $a$-th particle takes the form

$$
\frac{d p_{a \mu}(\sigma)}{d \sigma}=q_{a} \sum_{\substack{b \neq a \\ \eta_{a} \eta_{b}=1}} F_{\mu \nu}^{(b)}\left(r_{a}(\sigma)\right) V_{a}^{\nu}(\sigma) \simeq \frac{1}{\sigma^{2}} \sum_{\substack{b \neq a \\ \eta_{a} \eta_{b}=1}} \eta_{a} \eta_{b} \frac{q_{a} q_{b}}{4 \pi} \frac{V_{a} \cdot V_{b} V_{a \mu}+V_{b \mu}}{\left\{\left(V_{b} \cdot V_{a}\right)^{2}-1\right\}^{3 / 2}} .
$$

On the other hand we have

$$
\frac{d p_{a \mu}(\sigma)}{d \sigma}=\frac{m_{a}}{\eta_{a}} \frac{d^{2} r_{a \mu}}{d \sigma^{2}}=-\frac{m_{a}}{\eta_{a}} \frac{c_{a \mu}}{\sigma^{2}}
$$

where in the last step we used (3.3). Comparing (3.12), (3.13) we get

$$
c_{a}^{\mu}=-\frac{1}{m_{a}} \sum_{\substack{b \neq a \\ \eta_{a} \eta_{b}=1}} \eta_{b} \frac{q_{a} q_{b}}{4 \pi} \frac{V_{a} \cdot V_{b} V_{a}^{\mu}+V_{b}^{\mu}}{\left\{\left(V_{b} \cdot V_{a}\right)^{2}-1\right\}^{3 / 2}}=-\sum_{\substack{b \neq a \\ \eta_{a} \eta_{b}=1}} \frac{q_{a} q_{b}}{4 \pi} \frac{m_{b}^{2} p_{a} \cdot p_{b} p_{a}^{\mu}+m_{a}^{2} m_{b}^{2} p_{b}^{\mu}}{\left\{\left(p_{b} \cdot p_{a}\right)^{2}-m_{a}^{2} m_{b}^{2}\right\}^{3 / 2}},
$$

and

$$
c_{a}^{\mu} p_{a}^{\nu}-c_{a}^{\nu} p_{a}^{\mu}=-\sum_{\substack{b \neq a \\ \eta_{a} \eta_{b}=1}} \frac{q_{a} q_{b}}{4 \pi} \frac{m_{a}^{2} m_{b}^{2}\left\{p_{b}^{\mu} p_{a}^{\nu}-p_{b}^{\nu} p_{a}^{\mu}\right\}}{\left\{\left(p_{b} \cdot p_{a}\right)^{2}-m_{a}^{2} m_{b}^{2}\right\}^{3 / 2}} .
$$

Eqs. (3.5) and (3.6) now give ${ }^{4}$

$$
S_{\mathrm{em}}=\sum_{a} \frac{\varepsilon_{\mu} p_{a}^{\mu}}{p_{a} \cdot k} q_{a}-i \ln \omega^{-1} \sum_{a} \frac{q_{a} \varepsilon_{\mu} k_{\rho}}{p_{a} \cdot k} \sum_{\substack{b \neq a \\ \eta_{a} \eta_{b}=1}} \frac{q_{a} q_{b}}{4 \pi} \frac{m_{a}^{2} m_{b}^{2}\left\{p_{b}^{\rho} p_{a}^{\mu}-p_{b}^{\mu} p_{a}^{\rho}\right\}}{\left\{\left(p_{b} \cdot p_{a}\right)^{2}-m_{a}^{2} m_{b}^{2}\right\}^{3 / 2}},
$$

\footnotetext{
${ }^{4}$ Note that even if we assume that the logarithmic corrections to the trajectories are generated predominantly by electromagnetic interaction, the resulting acceleration can generate logarithmic corrections to the gravitational radiation during the scattering.
} 
and

$$
S_{\mathrm{gr}}=\sum_{a} \frac{\varepsilon_{\mu \nu} p_{a}^{\mu} p_{a}^{\nu}}{p_{a} \cdot k}-i \ln \omega^{-1} \sum_{a} \frac{\varepsilon_{\mu \nu} p_{a}^{\nu} k_{\rho}}{p_{a} . k} \sum_{\substack{b \neq a \\ \eta_{a} \eta_{b}=1}} \frac{q_{a} q_{b}}{4 \pi} \frac{m_{a}^{2} m_{b}^{2}\left\{p_{b}^{\rho} p_{a}^{\mu}-p_{b}^{\mu} p_{a}^{\rho}\right\}}{\left\{\left(p_{b} \cdot p_{a}\right)^{2}-m_{a}^{2} m_{b}^{2}\right\}^{3 / 2}} .
$$

\subsection{Effect of gravitational interactions}

Let us now suppose that the logarithmic correction to the trajectories arise due to gravitational interaction. We introduce the graviton field $h_{\mu \nu}$ and its trace reversed version $e_{\mu \nu}$ via the equations

$$
h_{\mu \nu} \equiv\left(g_{\mu \nu}-\eta_{\mu \nu}\right) / 2, \quad e_{\mu \nu}=h_{\mu \nu}-\frac{1}{2} \eta_{\mu \nu} h_{\rho}^{\rho} .
$$

Then the analog of (3.7) for the gravitational field produced at $x$ due to the $b$-th particle is

$$
e_{\mu \nu}^{(b)}(x)=\frac{1}{2 \pi} \int d \sigma m_{b} V_{b \mu}(\sigma) V_{b \nu}(\sigma) \delta_{+}\left(-\left(x-r_{b}(\sigma)\right)^{2}\right) .
$$

Using $r_{b}(\sigma)=V_{b} \sigma+\cdots$ we get the analog of (3.9)

$$
e_{\mu \nu}^{(b)}(x) \simeq \frac{1}{4 \pi} \frac{m_{b} V_{b \mu} V_{b \nu}}{\sqrt{\left(V_{b} \cdot x\right)^{2}+x^{2}}}
$$

The associated Christoffel symbol is given by, in the weak field approximation,

$$
\begin{aligned}
\Gamma_{\rho \tau}^{(b) \alpha}(x)= & -\frac{m_{b}}{4 \pi} \frac{1}{\left\{\left(V_{b} \cdot x\right)^{2}+x^{2}\right\}^{3 / 2}} \eta^{\alpha \mu}\left[\left\{V_{b \mu} V_{b \tau}+\frac{1}{2} \eta_{\mu \tau}\right\}\left\{x_{\rho}+V_{b} \cdot x V_{b \rho}\right\}\right. \\
& \left.+\left\{V_{b \mu} V_{b \rho}+\frac{1}{2} \eta_{\mu \rho}\right\}\left\{x_{\tau}+V_{b} . x V_{b \tau}\right\}-\left\{V_{b \rho} V_{b \tau}+\frac{1}{2} \eta_{\rho \tau}\right\}\left\{x_{\mu}+V_{b} . x V_{b \mu}\right\}\right] .
\end{aligned}
$$

From this we can write down the equation of motion of the $a$-th particle

$$
\begin{aligned}
\frac{d^{2} r_{a}^{\alpha}(\sigma)}{d \sigma^{2}} & =-\sum_{\substack{b \neq a \\
\eta_{a} \eta_{b}=1}} \Gamma_{\rho \tau}^{(b) \alpha}\left(r_{a}(\sigma)\right) V_{a}^{\rho}(\sigma) V_{a}^{\tau}(\sigma) \\
& \simeq-\eta_{a} \frac{1}{4 \pi \sigma^{2}} \sum_{\substack{b \neq a \\
\eta_{a} \eta_{b}=1}} m_{b} \frac{1}{\left\{\left(V_{b} \cdot V_{a}\right)^{2}-1\right\}^{3 / 2}}\left[-\frac{1}{2} V_{a}^{\alpha}+\frac{1}{2} V_{b}^{\alpha}\left\{2\left(V_{b} \cdot V_{a}\right)^{3}-3 V_{b} \cdot V_{a}\right\}\right] .
\end{aligned}
$$

On the other hand using (3.3) the left hand side is given by $-c_{a}^{\alpha} / \sigma^{2}$. This gives

$$
c_{a}^{\alpha}=\eta_{a} \frac{1}{4 \pi} \sum_{\substack{b \neq a \\ \eta_{a} \eta_{b}=1}} m_{b} \frac{1}{\left\{\left(V_{b} \cdot V_{a}\right)^{2}-1\right\}^{3 / 2}}\left\{-\frac{1}{2} V_{a}^{\alpha}+\frac{1}{2} V_{b}^{\alpha}\left(2\left(V_{b} \cdot V_{a}\right)^{3}-3 V_{b} \cdot V_{a}\right)\right\},
$$

and

$$
\begin{aligned}
c_{a}^{\rho} p_{a}^{\mu}-c_{a}^{\mu} p_{a}^{\rho} & =\frac{1}{8 \pi \sigma^{2}} \sum_{\substack{b \neq a \\
\eta_{a} \eta_{b}=1}} m_{a} m_{b} \frac{1}{\left\{\left(V_{b} \cdot V_{a}\right)^{2}-1\right\}^{3 / 2}}\left(V_{b}^{\rho} V_{a}^{\mu}-V_{b}^{\mu} V_{a}^{\rho}\right)\left\{2\left(V_{b} \cdot V_{a}\right)^{3}-3 V_{b} \cdot V_{a}\right\} \\
& =\frac{1}{8 \pi} \sum_{\substack{b \neq a \\
\eta_{a} \eta_{b}=1}} \frac{p_{b} \cdot p_{a}}{\left\{\left(p_{b} \cdot p_{a}\right)^{2}-m_{a}^{2} m_{b}^{2}\right\}^{3 / 2}}\left(p_{b}^{\rho} p_{a}^{\mu}-p_{b}^{\mu} p_{a}^{\rho}\right)\left\{2\left(p_{b} \cdot p_{a}\right)^{2}-3 m_{a}^{2} m_{b}^{2}\right\} .
\end{aligned}
$$


Substituting this into (3.5) and (3.6) we get, ${ }^{5}$ up to overall phases:

$$
\begin{aligned}
S_{\mathrm{em}}=\sum_{a} \frac{\varepsilon_{\mu} p_{a}^{\mu}}{p_{a} \cdot k} q_{a} & +\frac{i}{8 \pi} \ln \omega^{-1} \sum_{a} \frac{q_{a} \varepsilon_{\mu} k_{\rho}}{p_{a} \cdot k} \sum_{\substack{b \neq a \\
\eta_{a} \eta_{b}=1}} \frac{p_{b} \cdot p_{a}}{\left\{\left(p_{b} \cdot p_{a}\right)^{2}-m_{a}^{2} m_{b}^{2}\right\}^{3 / 2}}\left(p_{b}^{\rho} p_{a}^{\mu}-p_{b}^{\mu} p_{a}^{\rho}\right) \\
& \times\left\{2\left(p_{b} \cdot p_{a}\right)^{2}-3 m_{a}^{2} m_{b}^{2}\right\}
\end{aligned}
$$

and

$$
\begin{aligned}
S_{\mathrm{gr}}=\sum_{a} \frac{\varepsilon_{\mu \nu} p_{a}^{\mu} p_{a}^{\nu}}{p_{a} \cdot k} & +\frac{i}{8 \pi} \ln \omega^{-1} \sum_{a} \frac{\varepsilon_{\mu \nu} p_{a}^{\nu} k_{\rho}}{p_{a} \cdot k} \sum_{\substack{b \neq a \\
\eta_{a} \eta_{b}=1}} \frac{p_{b} \cdot p_{a}}{\left\{\left(p_{b} \cdot p_{a}\right)^{2}-m_{a}^{2} m_{b}^{2}\right\}^{3 / 2}}\left(p_{b}^{\rho} p_{a}^{\mu}-p_{b}^{\mu} p_{a}^{\rho}\right) \\
& \times\left\{2\left(p_{b} \cdot p_{a}\right)^{2}-3 m_{a}^{2} m_{b}^{2}\right\} .
\end{aligned}
$$

In this case we expect the wave-form of the gauge field/metric to also have an additional phase factor reflecting the effect of the gravitational drag on the soft particle due to the other particles. For this let us characterize the asymptotic trajectory of the soft particle as

$$
x^{\mu}(\tau)=n^{\mu} \tau+m^{\mu} \ln |\tau|,
$$

where $\tau$ is the affine parameter associated with the trajectory, $n=(1, \hat{n})$ is a null vector along the asymptotic direction of motion of the soft particle and $m^{\mu}$ is a four vector to be determined. Now substituting (3.27) into the equation of motion

$$
\frac{d^{2} x^{\mu}}{d \tau^{2}}=-\Gamma_{\nu \rho}^{\mu} \frac{d x^{\nu}}{d \tau} \frac{d x^{\rho}}{d \tau},
$$

and using the form (3.21) of $\Gamma_{\nu \rho}^{\mu}$, we get the following expression for $m^{\mu}$ by comparing the $1 / \tau^{2}$ terms on the two sides of the equations of motion:

$$
m^{\alpha}=-\frac{1}{4 \pi} \sum_{\substack{b \\ \eta_{b}=-1}} \frac{m_{b}}{\left|n \cdot V_{b}\right|^{3}} V_{b}^{\alpha}\left(V_{b} \cdot n\right)^{3}=\frac{1}{4 \pi} \sum_{\substack{b \\ \eta_{b}=-1}} m_{b} V_{b}^{\alpha}=-\frac{1}{4 \pi} \sum_{\substack{b \\ \eta_{b}=-1}} p_{b}^{\alpha} .
$$

Now eliminating $\tau$ in terms of $t \equiv x^{0}$ using (3.27), we can express (3.27) as

$$
x^{i}=n^{i} t+\left(m^{i}-n^{i} m^{0}\right) \ln |t|+\text { finite } .
$$

Therefore if we denote by $k=\left(k^{0}, k\right)=-\omega(1, \hat{n})$ the four momentum of the soft particle, the overall - sign reflecting the fact that it is an outgoing particle, the wave-function of the particle will be proportional to

$$
\exp \left[-i \vec{k} \cdot\left\{\vec{x}-\hat{n} t-\left(\vec{m}-\hat{n} m^{0}\right) \ln |t|\right\}\right]=\exp [-i \omega t+i \omega \hat{n} \cdot \vec{x}] \exp \left[i\left(\vec{k} \cdot \vec{m}+\omega m^{0}\right) \ln |t|\right]
$$

\footnotetext{
${ }^{5}$ Even if the logarithmic correction to the trajectory is generated by gravitational interaction, the particles can emit electromagnetic waves. This happens for example if we have a scattering of a charged particle and a neutral particle.
} 
The second factor can be regarded as an additional infrared divergent contribution to the soft factor. Using $|t| \sim R$ where $R$ is the distance of the soft particle from the scattering center, and eq. (3.29), we can express the second factor in (3.31) as

$$
\exp [i k \cdot m \ln R]=\exp \left[-\frac{i}{4 \pi} \ln R \sum_{\substack{b \\ \eta_{b}=-1}} k \cdot p_{b}\right] .
$$

Since this is a pure phase it does not affect the flux. However it does produce observable effect on the electromagnetic/gravitational wave-form [90].

It follows from the analysis of $[91,93,94]$ that the effect of gravitational backscattering of the soft photon/graviton actually converts $\ln R$ in (3.32) to $\ln (R \omega)$. This has been reviewed in [89]. It is natural to absorb this multiplicative factor in the wave-form into the definition of the soft factors. Expanding the exponential in a power series, picking up the term of order $\omega \ln (\omega R)$ in the expansion, and multiplying this by the leading soft factor, we get additional contributions to the soft photon and soft graviton factor at the subleading order

$$
\frac{i}{4 \pi}\left(\ln \omega^{-1}+\ln R^{-1}\right) S_{\mathrm{em}}^{(0)} \sum_{\substack{b \\ \eta_{b}=-1}} k \cdot p_{b}, \quad \text { and } \quad \frac{i}{4 \pi}\left(\ln \omega^{-1}+\ln R^{-1}\right) S_{\mathrm{gr}}^{(0)} \sum_{\substack{b \\ \eta_{b}=-1}} k \cdot p_{b} .
$$

Adding these to (3.25) and (3.26) we get the net soft factors to be

$$
\begin{aligned}
S_{\mathrm{em}}= & \sum_{a} \frac{\varepsilon_{\mu} p_{a}^{\mu}}{p_{a} \cdot k} q_{a}+\frac{i}{4 \pi}\left(\ln \omega^{-1}+\ln R^{-1}\right) \sum_{\substack{b \\
\eta_{b}=-1}} k \cdot p_{b} \sum_{a} \frac{\varepsilon_{\mu} p_{a}^{\mu}}{p_{a} \cdot k} q_{a} \\
& +\frac{i}{8 \pi} \ln \omega^{-1} \sum_{a} \frac{q_{a} \varepsilon_{\mu} k_{\rho}}{p_{a} \cdot k} \sum_{\substack{b \neq a \\
\eta_{a} \eta_{b}=1}} \frac{p_{b} \cdot p_{a}}{\left\{\left(p_{b} \cdot p_{a}\right)^{2}-m_{a}^{2} m_{b}^{2}\right\}^{3 / 2}}\left(p_{b}^{\rho} p_{a}^{\mu}-p_{b}^{\mu} p_{a}^{\rho}\right)\left\{2\left(p_{b} \cdot p_{a}\right)^{2}-3 m_{a}^{2} m_{b}^{2}\right\},
\end{aligned}
$$

and

$$
\begin{aligned}
S_{\mathrm{gr}}= & \sum_{a} \frac{\varepsilon_{\mu \nu} p_{a}^{\mu} p_{a}^{\nu}}{p_{a} \cdot k}+\frac{i}{4 \pi}\left(\ln \omega^{-1}+\ln R^{-1}\right) \sum_{\substack{b \\
\eta_{b}=-1}} k \cdot p_{b} \sum_{a} \frac{\varepsilon_{\mu \nu} p_{a}^{\mu} p_{a}^{\nu}}{p_{a} \cdot k} \\
& +\frac{i}{8 \pi} \ln \omega^{-1} \sum_{a} \frac{\varepsilon_{\mu \nu} p_{a}^{\nu} k_{\rho}}{p_{a} \cdot k} \sum_{\substack{b \neq a \\
\eta_{a} \eta_{b}=1}} \frac{p_{b} \cdot p_{a}}{\left\{\left(p_{b} \cdot p_{a}\right)^{2}-m_{a}^{2} m_{b}^{2}\right\}^{3 / 2}}\left(p_{b}^{\rho} p_{a}^{\mu}-p_{b}^{\mu} p_{a}^{\rho}\right)\left\{2\left(p_{b} \cdot p_{a}\right)^{2}-3 m_{a}^{2} m_{b}^{2}\right\}
\end{aligned}
$$

\subsection{Effect of electromagnetic and gravitational interactions}

We now combine the results of last two subsections to write down the general expression for the soft factor when both gravitational interaction and electromagnetic interactions are responsible for the logarithmic corrections to the trajectory. The logarithmic terms get 
added up, yielding the results:

$$
\begin{aligned}
S_{\mathrm{em}}= & \sum_{a} \frac{\varepsilon_{\mu} p_{a}^{\mu}}{p_{a} \cdot k} q_{a}+\frac{i}{4 \pi}\left(\ln \omega^{-1}+\ln R^{-1}\right) \sum_{\substack{b \\
\eta_{b}=-1}} k \cdot p_{b} \sum_{a} \frac{\varepsilon_{\mu} p_{a}^{\mu}}{p_{a} \cdot k} q_{a} \\
& -i \ln \omega^{-1} \sum_{a} \frac{q_{a} \varepsilon_{\mu} k_{\rho}}{p_{a} \cdot k} \sum_{\substack{b \neq a \\
\eta_{a} \eta_{b}=1}} \frac{q_{a} q_{b}}{4 \pi} \frac{m_{a}^{2} m_{b}^{2}\left\{p_{b}^{\rho} p_{a}^{\mu}-p_{b}^{\mu} p_{a}^{\rho}\right\}}{\left\{\left(p_{b} \cdot p_{a}\right)^{2}-m_{a}^{2} m_{b}^{2}\right\}^{3 / 2}} \\
& +\frac{i}{8 \pi} \ln \omega^{-1} \sum_{a} \frac{q_{a} \varepsilon_{\mu} k_{\rho}}{p_{a} \cdot k} \sum_{\substack{b \neq a \\
\eta_{a} \eta_{b}=1}} \frac{p_{b} \cdot p_{a}}{\left\{\left(p_{b} \cdot p_{a}\right)^{2}-m_{a}^{2} m_{b}^{2}\right\}^{3 / 2}}\left(p_{b}^{\rho} p_{a}^{\mu}-p_{b}^{\mu} p_{a}^{\rho}\right)\left\{2\left(p_{b} \cdot p_{a}\right)^{2}-3 m_{a}^{2} m_{b}^{2}\right\},
\end{aligned}
$$

and

$$
\begin{aligned}
S_{\mathrm{gr}}= & \sum_{a} \frac{\varepsilon_{\mu \nu} p_{a}^{\mu} p_{a}^{\nu}}{p_{a} \cdot k}+\frac{i}{4 \pi}\left(\ln \omega^{-1}+\ln R^{-1}\right) \sum_{\substack{b \\
\eta_{b}=-1}} k \cdot p_{b} \sum_{a} \frac{\varepsilon_{\mu \nu} p_{a}^{\mu} p_{a}^{\nu}}{p_{a} \cdot k} \\
& -i \ln \omega^{-1} \sum_{a} \frac{\varepsilon_{\mu \nu} p_{a}^{\nu} k_{\rho}}{p_{a} \cdot k} \sum_{\substack{b \neq a \\
\eta_{a} \eta_{b}=1}} \frac{q_{a} q_{b}}{4 \pi} \frac{m_{a}^{2} m_{b}^{2}\left\{p_{b}^{\rho} p_{a}^{\mu}-p_{b}^{\mu} p_{a}^{\rho}\right\}}{\left\{\left(p_{b} \cdot p_{a}\right)^{2}-m_{a}^{2} m_{b}^{2}\right\}^{3 / 2}} \\
& +\frac{i}{8 \pi} \ln \omega^{-1} \sum_{a} \frac{\varepsilon_{\mu \nu} p_{a}^{\nu} k_{\rho}}{p_{a} \cdot k} \sum_{\substack{b \neq a \\
\eta_{a} \eta_{b}=1}} \frac{p_{b} \cdot p_{a}}{\left\{\left(p_{b} \cdot p_{a}\right)^{2}-m_{a}^{2} m_{b}^{2}\right\}^{3 / 2}}\left(p_{b}^{\rho} p_{a}^{\mu}-p_{b}^{\mu} p_{a}^{\rho}\right)\left\{2\left(p_{b} \cdot p_{a}\right)^{2}-3 m_{a}^{2} m_{b}^{2}\right\} .
\end{aligned}
$$

These reproduce (2.4) and (2.6) respectively.

Note that the soft factors given in (3.36) and (3.37) depend only on the charges and momenta carried by the external states. Therefore these can be reinterpreted as multiplicative soft factors in the full quantum theory - since there is no angular momentum there is no derivative with respect to the external momenta. In the next few sections we shall carry out some explicit quantum computations to examine to what extent this holds.

\section{How to treat momentum conservation and infrared divergences}

In quantum theory, single soft theorem is expected to relate an amplitude $\Gamma^{(n, 1)}$ with $n$ finite energy external states carrying momenta $p_{1}, \cdots p_{n}$ and one soft particle of momentum $k$ to an amplitude $\Gamma^{(n)}$ with just $n$ finite energy external states carrying momenta $p_{1}, \cdots p_{n}$. This relation takes the form

$$
\Gamma^{(n, 1)}\left(p_{1}, \cdots p_{n}, k\right) \simeq S\left(\varepsilon, k ;\left\{p_{a}\right\}\right) \Gamma^{(n)}\left(p_{1}, \cdots p_{n}\right),
$$

where $S\left(\varepsilon, k ;\left\{p_{a}\right\}\right)$ is the soft factor $S_{\mathrm{em}}$ or $S_{\mathrm{gr}}$. There is however a potential problem. While the amplitude $\Gamma^{(n, 1)}$ has momentum conservation $\sum_{a} p_{a}+k=0$, the amplitude $\Gamma^{(n)}$ has momentum conservation $\sum_{a} p_{a}=0$. Therefore we cannot keep the $p_{a}$ 's and $k$ as independent variables in (4.1). Usually this problem is overcome by including the momentum conserving delta-functions in the definition of the amplitudes $\Gamma^{(n, 1)}$ and $\Gamma^{(n)}$ and 
treating (4.1) as a relation between distributions. The soft factor $S\left(\varepsilon, k ;\left\{p_{a}\right\}\right)$ appearing in (4.1) is treated as a differential operator that also acts on the delta function and generates the Taylor series expansion of $\delta\left(\sum_{a} p_{a}+k\right)$ in power series of the momentum $k$ of the soft particle. The subleading term in this expansion, given by $k^{\mu}\left\{\partial / \partial p_{b}^{\mu}\right\} \delta\left(\sum_{a} p_{a}\right)$ for any $b$, is included in the full subleading soft theorem in dimensions $D>4$. However since in $D=4$ we only analyze subleading terms containing $\ln \omega^{-1}$ factors, the term proportional to derivative of the delta function will not appear in our analysis.

In four space-time dimensions there are additional issues due to infrared divergence. Both the amplitudes $\Gamma^{(n, 1)}$ and $\Gamma^{(n)}$ have infrared divergences which can be represented as overall multiplicative factors multiplying infrared finite amplitudes. For electromagnetic interactions these factors are common and can be factored out of the amplitudes but for gravity there is a residual infrared divergent factor in $\Gamma^{(n, 1)}$ besides the ones that appear in $\Gamma^{(n)}$. In any case we shall denote by $\exp [K]$ the infrared divergent factor of $\Gamma^{(n)}$ and define regulated amplitudes via the relation:

$$
\Gamma^{(n)}=\exp [K] \Gamma_{\text {reg }}^{(n)}, \quad \Gamma^{(n, 1)}=\exp [K] \Gamma_{\text {reg }}^{(n, 1)} .
$$

$K$ is in general a function of the momenta $p_{a}$ of the finite energy particles. This makes $\Gamma_{\text {reg }}^{(n)}$ free from infrared divergences, but $\Gamma_{\text {reg }}^{(n, 1)}$ still contains some residual infrared divergences for gravitational interaction. Eq. (4.1) is now replaced by ${ }^{6}$

$$
\Gamma_{\text {reg }}^{(n, 1)}\left(p_{1}, \cdots p_{n}, k\right) \simeq S\left(\varepsilon, k ;\left\{p_{a}\right\}\right) \Gamma_{\text {reg }}^{(n)}\left(p_{1}, \cdots p_{n}\right) .
$$

The residual infrared divergences in $\Gamma_{\text {reg }}^{(n, 1)}$ will be reflected in the infrared divergent contributions to $S\left(\varepsilon, k ;\left\{p_{a}\right\}\right)$.

There is however a potential ambiguity in the definition of $\Gamma_{\text {reg }}^{(n, 1)}$ and hence of $S\left(\varepsilon, k ;\left\{p_{a}\right\}\right)$. This is due to the fact that in the definition of $K$ we can add a term of the form $Q \cdot \sum_{a} p_{a}$ for any vector $Q$ (which could be a function of the $p_{a}$ 's) since by the momentum conserving delta function in $\Gamma^{(n)}, \sum_{a} p_{a}$ vanishes. However addition of such a term changes the definition of $\Gamma_{\text {reg }}^{(n, 1)}$ in (4.2) by a multiplicative factor of $\exp [k . Q]$ since the momentum conserving delta function in $\Gamma^{(n, 1)}$ gives $k+\sum_{a} p_{a}=0$. This has the effect of multiplying $S\left(\varepsilon, k ;\left\{p_{a}\right\}\right)$ by $\exp [k \cdot Q]$. Expanding $\exp (k \cdot Q)$ as $(1+k . Q)$ we see that the additional contribution appears at the subleading order, and has the form of $k . Q$ multiplying the leading soft factor. It does not affect the $\ln \omega^{-1}$ terms that we are after since the leading soft factor has no $\ln \omega^{-1}$ term and $Q$ is $\omega$ independent. However this can affect the genuine infrared divergent terms proportional to $\ln R$ in the expression for $\Gamma_{\text {reg }}^{(n, 1)}$, since in the definition of $Q$ we can include terms proportional to $\ln R$. Choosing $Q=-U \ln R$ for some vector $U$ constructed from the $p_{a}$ 's amounts to having an additive contribution to $S^{(1)}$ of the form

$$
-\ln R k \cdot U S^{(0)}\left(\varepsilon, k ;\left\{p_{a}\right\}\right) .
$$

\footnotetext{
${ }^{6}$ The situation here is somewhat different from the one in [41]. Since the logarithmic term in $S\left(\varepsilon, k ;\left\{p_{a}\right\}\right)$ that we are after is being represented as a multiplicative factor instead of a differential operator, the infrared divergent factor on the right hand side can be moved past $S$ to the extreme left.
} 


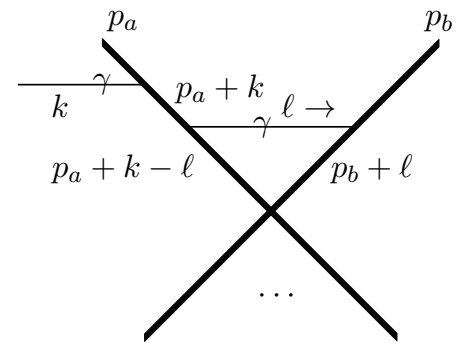

(a)

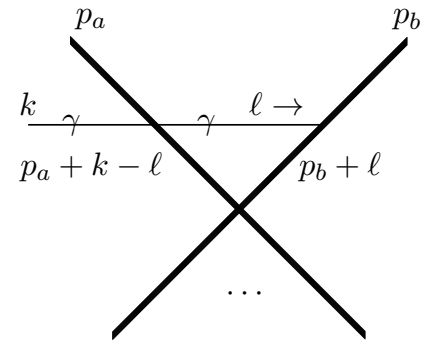

(b)

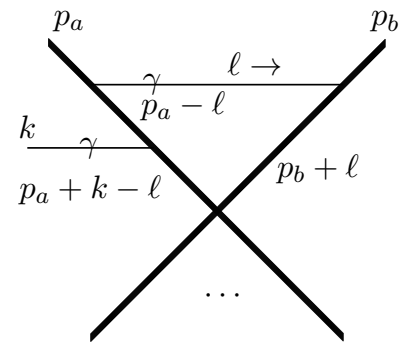

(c)

Figure 1. One loop contribution to $\Gamma^{(n, 1)}$ involving internal photon line connecting two different legs. The thick lines represent scalar particles and the thin lines carrying the symbol $\gamma$ represent photons. There are other diagrams related to this by permutations of the external scalar particles.

\section{Soft photon theorem in scalar QED}

Consider a theory containing a $\mathrm{U}(1)$ gauge field $A_{\mu}$ and $n$ scalars $\phi_{1}, \cdots \phi_{n}$ of masses $m_{1}, \cdots m_{n}$ and carrying $\mathrm{U}(1)$ charges $q_{1}, \cdots q_{n}$, satisfying $\sum_{a=1}^{n} q_{a}=0$. We further assume that there is a non-derivative contact interaction between the $n$-scalars. Then the relevant part of the action takes the form

$$
\begin{gathered}
\int d^{4} x\left[-\frac{1}{4} F_{\mu \nu} F^{\mu \nu}-\sum_{a=1}^{n}\left\{\left(\partial_{\mu} \phi_{a}^{*}+i q_{a} A_{\mu} \phi_{a}^{*}\right)\left(\partial^{\mu} \phi_{a}-i q_{a} A^{\mu} \phi_{a}\right)+m_{a}^{2} \phi_{a}^{*} \phi_{a}\right\}\right. \\
\left.+\lambda \phi_{1} \cdots \phi_{n}+\lambda \phi_{1}^{*} \cdots \phi_{n}^{*}\right] .
\end{gathered}
$$

We consider in this theory an amplitude with one external outgoing photon of momentum $k$ and $n$ external states corresponding to the fields $\phi_{1}, \cdots \phi_{n}$, carrying momenta $p_{1}, \cdots p_{n}$. All momenta are counted as positive if ingoing so that if the $a$-th particle is outgoing it will have negative $p_{a}^{0}$. Our goal will be to analyze this amplitude at one loop order, involving an internal photon connecting two matter lines. The relevant diagrams have been shown in figures 1 and 2 . We denote by $\Gamma^{(n, 1)}$ the sum over tree and one loop contribution to this amplitude. $\Gamma^{(n)}$ will denote the amplitude without the external soft photon to one loop order. One loop contribution to $\Gamma^{(n)}$ has been shown in figure 3.

In our analysis we shall ignore graphs with self energy insertions on external legs and assume that we follow on-shell renormalization so that the mass parameters appearing in the tree level propagators are the physical masses. The wave-function renormalization of the external scalars cancel between $\Gamma^{(n)}$ and $\Gamma^{(n, 1)}$.

We shall use Feynman gauge and decompose the photon propagator of momentum $\ell$, connecting the leg $a$ to the leg $b$ for $b \neq a$, with $\ell$ flowing from the $a$-th leg to the $b$-th leg, as [99]

$$
-i \frac{\eta^{\mu \nu}}{\ell^{2}-i \epsilon}=-\frac{i}{\ell^{2}-i \epsilon}\left\{K_{(a b)}^{\mu \nu}+G_{(a b)}^{\mu \nu}\right\}
$$

where,

$$
K_{(a b)}^{\mu \nu}=\ell^{\mu} \ell^{\nu} \frac{\left(2 p_{a}-\ell\right) \cdot\left(2 p_{b}+\ell\right)}{\left(2 p_{a} \cdot \ell-\ell^{2}+i \epsilon\right)\left(2 p_{b} \cdot \ell+\ell^{2}-i \epsilon\right)}, \quad G_{(a b)}^{\mu \nu}=\eta^{\mu \nu}-K_{(a b)}^{\mu \nu} .
$$



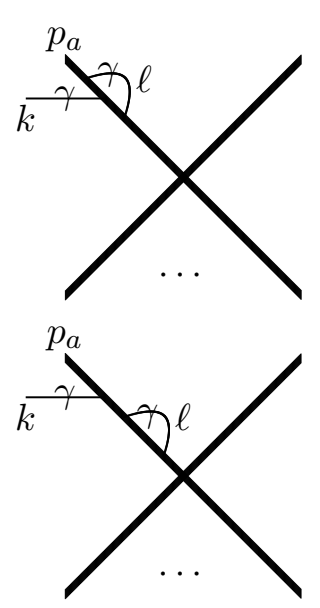
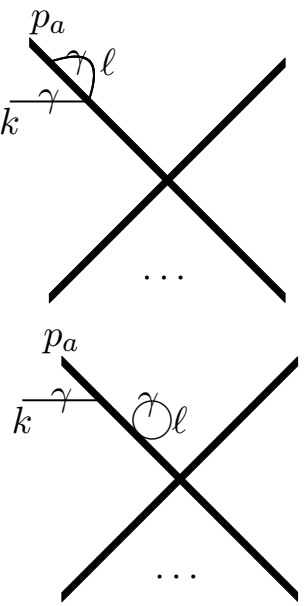
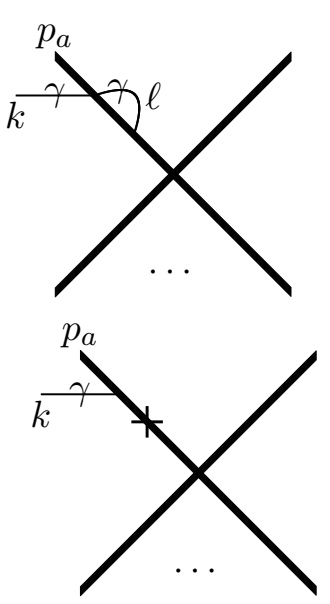

Figure 2. One loop contribution to $\Gamma^{(n, 1)}$ involving internal photon line connecting two different points on the same leg. There are other diagrams related to this by permutations of the external scalar particles. In the last term the + on the scalar line represents a counterterm associated with mass renormalization that has to be adjusted to cancel the net contribution proportional to $1 /\left(p_{a} \cdot k\right)^{2}$.

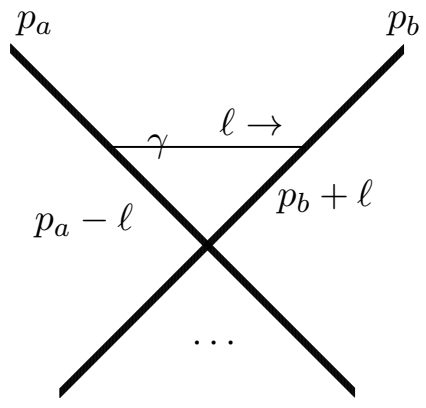

Figure 3. One loop contribution to $\Gamma^{(n)}$. There are other diagrams related to this by permutations of the external scalar particles.

Note that $p_{a}$ and $p_{b}$ refer to the external momenta flowing into the legs $a$ and $b$, and not necessarily the momenta of the lines to which the photon propagator attaches (which may have additional contribution from external soft momentum, e.g. in figures 1 (a)). $\ell$ denotes the momentum flowing from leg $a$ to leg $b$. For $a=b$ we do not carry out any decomposition.

Since the K-photon polarization is proportional to $\ell^{\mu} \ell^{\nu}$, it is pure gauge. This allows us to sum over K-photon insertions using Ward identities

$$
\frac{-i}{p_{c}^{2}+m_{c}^{2}} \ell^{\mu} i q_{c}\left(2 p_{c \mu}+\ell_{\mu}\right) \frac{-i}{\left(p_{c}+\ell\right)^{2}+m_{c}^{2}}=-q_{c}\left[\frac{-i}{\left(p_{c}+\ell\right)^{2}+m_{c}^{2}}-\frac{-i}{p_{c}^{2}+m_{c}^{2}}\right]
$$

and

$$
q_{c}\left[i q_{c} \varepsilon \cdot\left(2 p_{c}+2 \ell+k\right)-i q_{c} \varepsilon \cdot\left(2 p_{c}+k\right)\right]-2 i q_{c}^{2} \varepsilon \cdot \ell=0,
$$

whose diagrammatic representations have been shown in figure 4. Sum over all insertions 


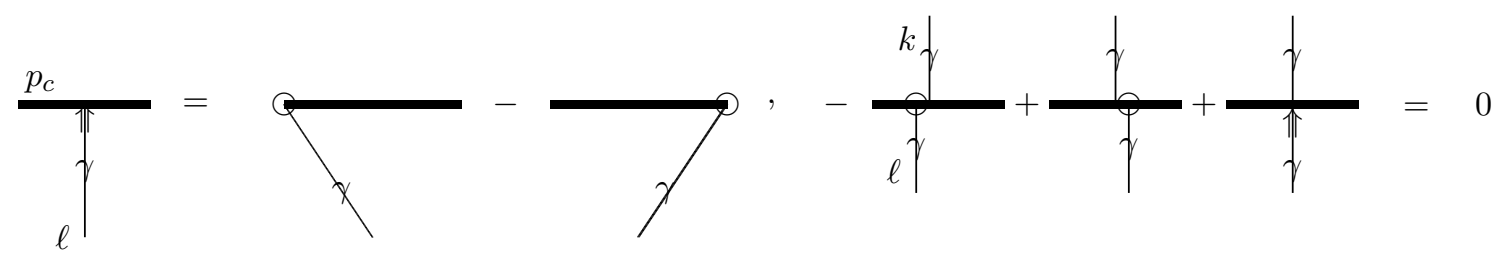

Figure 4. Diagrammatic representations of (5.4) and (5.5). The arrow on the photon line represents that the polarization of the photon is taken to be equal to the momentum entering the vertex. The circle denotes a simple vertex $-q_{c}$ with the polarization of the incoming photon stripped off.

of the K-photons to either $\Gamma^{(n)}$ or $\Gamma^{(n, 1)}$ produces an exponential factor [99]

$$
\exp \left[i \sum_{a<b} q_{a} q_{b} \int \frac{d^{4} \ell}{(2 \pi)^{4}} \frac{1}{\ell^{2}-i \epsilon} \frac{\left(2 p_{a}-\ell\right) \cdot\left(2 p_{b}+\ell\right)}{\left(2 p_{a} \cdot \ell-\ell^{2}+i \epsilon\right)\left(2 p_{b} \cdot \ell+\ell^{2}-i \epsilon\right)}\right] .
$$

Therefore we may write

$$
\begin{aligned}
& \Gamma^{(n)}=\exp \left[K_{\mathrm{em}}\right]\left\{\Gamma_{\text {tree }}^{(n)}+\Gamma_{\mathrm{G}}^{(n)}\right\}, \quad \Gamma^{(n, 1)}=\exp \left[K_{\mathrm{em}}\right]\left\{\Gamma_{\text {tree }}^{(n, 1)}+\Gamma_{\mathrm{G}}^{(n, 1)}+\Gamma_{\text {self }}^{(n, 1)}\right\}, \\
& K_{\mathrm{em}} \equiv \frac{i}{2} \sum_{\substack{a, b \\
b \neq a}} q_{a} q_{b} \int \frac{d^{4} \ell}{(2 \pi)^{4}} \frac{1}{\ell^{2}-i \epsilon} \frac{\left(2 p_{a}-\ell\right) \cdot\left(2 p_{b}+\ell\right)}{\left(2 p_{a} \cdot \ell-\ell^{2}+i \epsilon\right)\left(2 p_{b} \cdot \ell+\ell^{2}-i \epsilon\right)},
\end{aligned}
$$

where $\Gamma_{\mathrm{G}}^{(n)}$ and $\Gamma_{\mathrm{G}}^{(n, 1)}$ are computed by replacing the internal photons by the G-photons in figures 3 and 1 respectively and $\Gamma_{\text {self }}^{(n, 1)}$ denotes the sum of diagrams in figure 2 for which we use the full photon propagator. Therefore a relation of the form $\Gamma^{(n, 1)}=S_{\mathrm{em}} \Gamma^{(n)}$ takes the form

$$
\Gamma_{\text {tree }}^{(n, 1)}+\Gamma_{\mathrm{G}}^{(n, 1)}+\Gamma_{\text {self }}^{(n, 1)}=S_{\mathrm{em}}\left\{\Gamma_{\text {tree }}^{(n)}+\Gamma_{\mathrm{G}}^{(n)}\right\}
$$

Now it is easy to see that figure 3 vanishes when we replace the internal photon by G-photon. Therefore $\Gamma_{\mathrm{G}}^{(n)}=0$, and we have: ${ }^{7}$

$$
\Gamma_{\text {tree }}^{(n)}+\Gamma_{\mathrm{G}}^{(n)}=\Gamma_{\text {tree }}^{(n)}=i \lambda .
$$

If we write $S_{\mathrm{em}}=S_{\mathrm{em}}^{(0)}+S_{\mathrm{em}}^{(1)}$ where $S_{\mathrm{em}}^{(0)}$ is the leading soft factor $\sum_{a=1}^{n} q_{a} \varepsilon \cdot p_{a} / k \cdot p_{a}$ and $S_{\mathrm{em}}^{(1)}$ is the subleading multiplicative factor containing logarithmic terms, then eq. (5.8) can be written as

$$
\Gamma_{\text {tree }}^{(n, 1)}+\Gamma_{\mathrm{G}}^{(n, 1)}+\Gamma_{\mathrm{self}}^{(n, 1)}=i \lambda \sum_{a=1}^{n} q_{a} \frac{\varepsilon \cdot p_{a}}{k \cdot p_{a}}+i \lambda S_{\mathrm{em}}^{(1)},
$$

to one loop order. Now $\Gamma_{\text {tree }}^{(n, 1)}$ is equal to the first term on the right hand side up to terms involving Taylor series expansion of the momentum conserving delta function in powers of

\footnotetext{
${ }^{7}$ Note that we are not explicitly writing the momentum conserving delta function, but are implicitly assuming that both sides of (5.8) are multiplied by the appropriate delta functions. We also implicitly assume that the delta function $\delta\left(\sum_{a} p_{a}+k\right)$ on the left hand side has been expanded in a power series in $k$.
} 
$k$, but the latter are subleading contributions without any logarithmic terms and can be ignored in our analysis. Therefore (5.10) can be rewritten as:

$$
\Gamma_{\mathrm{self}}^{(n, 1)}+\Gamma_{\mathrm{G}}^{(n, 1)}=i \lambda S_{\mathrm{em}}^{(1)}
$$

This is a simple algorithm for determination of $S_{\mathrm{em}}^{(1)}$.

Therefore we need to focus on the evaluation of the one loop contribution to $\Gamma_{\mathrm{G}}^{(n, 1)}$ and $\Gamma_{\text {self }}^{(n, 1)}$ by summing the diagrams in figures 1 and 2 , with the internal photon replaced by G-photon in figure 1. We first consider the diagrams in figure 1. It is easy to see that the G-photon contribution to figure 1(c) vanishes. Therefore we need to focus on figures 1(a) and (b). The contribution from figure 1 (a) is given by

$$
\begin{aligned}
\mathcal{I}_{1}=\lambda q_{a}^{2} q_{b} \frac{\epsilon \cdot p_{a}}{k \cdot p_{a}} & \int \frac{d^{4} \ell}{(2 \pi)^{4}}\left[2 k \cdot\left(2 p_{b}+\ell\right)-\frac{2 k \cdot \ell\left(2 p_{a}-\ell\right) \cdot\left(2 p_{b}+\ell\right)}{\left(2 p_{a} \cdot \ell-\ell^{2}+i \epsilon\right)}\right] \\
& \times \frac{1}{\ell^{2}-i \epsilon} \frac{1}{2 p_{a} \cdot(k-\ell)+(k-\ell)^{2}-i \epsilon} \frac{1}{2 p_{b} \cdot \ell+\ell^{2}-i \epsilon}
\end{aligned}
$$

and the contribution from figure 1(b) is given by

$$
\begin{aligned}
\mathcal{I}_{2}=-\lambda q_{a}^{2} q_{b} \int & \frac{d^{4} \ell}{(2 \pi)^{4}}\left[2 \epsilon \cdot\left(2 p_{b}+\ell\right)-\frac{2 \epsilon \cdot \ell\left(2 p_{a}-\ell\right) \cdot\left(2 p_{b}+\ell\right)}{\left(2 p_{a} \cdot \ell-\ell^{2}+i \epsilon\right)}\right] \\
& \times \frac{1}{\ell^{2}-i \epsilon} \frac{1}{2 p_{a} \cdot(k-\ell)+(k-\ell)^{2}-i \epsilon} \frac{1}{2 p_{b} \cdot \ell+\ell^{2}-i \epsilon}
\end{aligned}
$$

Both $\mathcal{I}_{1}$ and $\mathcal{I}_{2}$ are infrared finite since for small $\ell$ the integrands diverge as $1 / \ell^{3}$. The terms involving logarithm of $k$ come from the region of $\ell$ integration where the components $\left|\ell^{\mu}\right|$ are large compared to $\omega \equiv k_{0}$ but small compared to the $p_{a}$ 's. In this range we can approximate $\mathcal{I}_{1}$ and $\mathcal{I}_{2}$ as

$$
\begin{aligned}
\mathcal{I}_{1} & \simeq-\lambda q_{a}^{2} q_{b} \frac{\epsilon \cdot p_{a}}{k \cdot p_{a}} \int_{\mathrm{reg}} \frac{d^{4} \ell}{(2 \pi)^{4}}\left[k \cdot p_{b}-\frac{k \cdot \ell p_{a} \cdot p_{b}}{p_{a} \cdot \ell+i \epsilon}\right] \frac{1}{\ell^{2}-i \epsilon} \frac{1}{p_{a} \cdot \ell+i \epsilon} \frac{1}{p_{b} \cdot \ell-i \epsilon} \\
& =-\lambda q_{a}^{2} q_{b} \frac{\epsilon \cdot p_{a}}{k \cdot p_{a}}\left[k \cdot p_{b}+p_{a} \cdot p_{b} k^{\mu} \frac{\partial}{\partial p_{a}^{\mu}}\right] \int_{\mathrm{reg}} \frac{d^{4} \ell}{(2 \pi)^{4}} \frac{1}{\ell^{2}-i \epsilon} \frac{1}{p_{a} \cdot \ell+i \epsilon} \frac{1}{p_{b} \cdot \ell-i \epsilon},
\end{aligned}
$$

and

$$
\begin{aligned}
\mathcal{I}_{2} & \simeq \lambda q_{a} q_{a} q_{b} \int_{\mathrm{reg}} \frac{d^{4} \ell}{(2 \pi)^{4}}\left[\epsilon \cdot p_{b}-\frac{\epsilon \cdot \ell p_{a} \cdot p_{b}}{p_{a} \cdot \ell+i \epsilon}\right] \frac{1}{\ell^{2}-i \epsilon} \frac{1}{p_{a} \cdot \ell+i \epsilon} \frac{1}{p_{b} \cdot \ell-i \epsilon} \\
& =\lambda q_{a}^{2} q_{b}\left[\epsilon \cdot p_{b}+p_{a} \cdot p_{b} \epsilon^{\mu} \frac{\partial}{\partial p_{a}^{\mu}}\right] \int_{\mathrm{reg}} \frac{d^{4} \ell}{(2 \pi)^{4}} \frac{1}{\ell^{2}-i \epsilon} \frac{1}{p_{a} \cdot \ell+i \epsilon} \frac{1}{p_{b} \cdot \ell-i \epsilon},
\end{aligned}
$$

where the subscript reg indicates that the integration needs to be carried out over the region where $\left|\ell^{\mu}\right|$ is large compared to $\omega$ but small compared to the energies of the finite energy particles. Adding $\mathcal{I}_{1}$ and $\mathcal{I}_{2}$ and summing over $a, b$ we get the total contribution to 


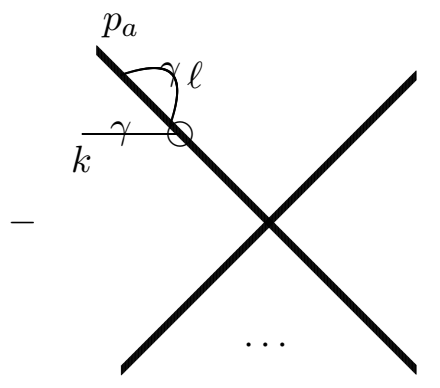

Figure 5. Sum of the first four diagrams in figure 2 with $\varepsilon$ replaced by $k$.

$\Gamma_{\mathrm{G}}^{(n, 1)}$ to one loop order:

$$
\begin{aligned}
\Gamma_{G}^{(n, 1)}= & -\lambda \sum_{\substack{a, b \\
b \neq a}}\left(q_{a}\right)^{2} q_{b}\left[\frac{\epsilon \cdot p_{a}}{k \cdot p_{a}} \quad k \cdot p_{b}+\frac{\epsilon \cdot p_{a}}{k \cdot p_{a}} p_{a} \cdot p_{b} k^{\mu} \frac{\partial}{\partial p_{a}^{\mu}}-\epsilon \cdot p_{b}-p_{a} \cdot p_{b} \epsilon^{\mu} \frac{\partial}{\partial p_{a}^{\mu}}\right] \\
& \int_{\text {reg }} \frac{d^{4} \ell}{(2 \pi)^{4}} \frac{1}{\ell^{2}-i \epsilon} \frac{1}{p_{a} \cdot \ell+i \epsilon} \frac{1}{p_{b} \cdot \ell-i \epsilon} \\
= & -\lambda \sum_{\substack{a, b \\
b \neq a}}\left(q_{a}\right)^{2} q_{b} \frac{\varepsilon_{\mu} k_{\nu}}{p_{a} \cdot k}\left\{p_{a}^{\mu} \frac{\partial}{\partial p_{a \nu}}-p_{a}^{\nu} \frac{\partial}{\partial p_{a \mu}}\right\} \int_{\mathrm{reg}} \frac{d^{4} \ell}{(2 \pi)^{4}} \frac{1}{\ell^{2}-i \epsilon} \frac{p_{a} \cdot p_{b}}{\left(p_{a} \cdot \ell+i \epsilon\right)\left(p_{b} \cdot \ell-i \epsilon\right)} .
\end{aligned}
$$

The contribution to $\Gamma_{\text {self }}^{(n, 1)}$ from figure 2 can be analyzed using the following indirect method. First of all we note that the net dependence on $\varepsilon$ and $k$ from the first four diagrams must be of the form $\varepsilon \cdot p_{a} f\left(p_{a} . k\right)$ for some function $f$. To determine $f$, we can set $\varepsilon=k$ and sum over all insertions of the external photon using the Ward identities shown in figure 4. The final result, given in figure 5, has the form:

$$
\frac{C_{1}}{p_{a} \cdot k}
$$

for some constant $C_{1}$. Therefore we get

$$
p_{a} \cdot k f\left(p_{a} \cdot k\right)=\frac{C_{1}}{p_{a} \cdot k} \quad \Rightarrow \quad f\left(p_{a} \cdot k\right)=\frac{C_{1}}{\left(p_{a} \cdot k\right)^{2}} .
$$

The fifth and sixth diagrams also have the form

$$
\frac{C_{2}}{\left(p_{a} \cdot k\right)^{2}} \text { and } \frac{C_{3}}{\left(p_{a} \cdot k\right)^{2}},
$$

for appropriate constants $C_{2}$ and $C_{3}$. Now since we are using on-shell renormalization the counterterm proportional to $C_{3}$ is to be adjusted precisely so that the net contribution proportional to $1 /\left(p_{a} \cdot k\right)^{2}$ vanishes. Therefore we must choose $C_{3}=-C_{1}-C_{2}$, and the total contribution to $\Gamma_{\text {self }}^{(n, 1)}$ from all the diagrams in figure 2 vanishes. We have verified this by explicitly computing the Feynman diagrams in figure 2 . 
From (5.11) we now see that the net contribution to the logarithmic terms in $S_{\mathrm{em}}^{(1)}$ is obtained by dividing $\Gamma_{\mathrm{G}}^{(n, 1)}$ given in (5.16) by $i \lambda$. This can be written as

$$
S_{\mathrm{em}}^{(1)}=\sum_{c} q_{c} \frac{\varepsilon_{\mu} k_{\nu}}{p_{c} . k}\left\{p_{c}^{\mu} \frac{\partial}{\partial p_{c \nu}}-p_{c}^{\nu} \frac{\partial}{\partial p_{c \mu}}\right\} K_{\mathrm{em}}^{\mathrm{reg}},
$$

where $K_{\mathrm{em}}^{\mathrm{reg}}$ is the factor $K_{\mathrm{em}}$ defined in (5.7) with the understanding that integration over the loop momentum $\ell$ will run over the range where $\left|\ell^{\mu}\right|$ is larger than $\omega$ but small compared to the momenta of the finite energy external states:

$$
K_{\mathrm{em}}^{\mathrm{reg}} \equiv \frac{i}{2} \sum_{\substack{a, b \\ b \neq a}} q_{a} q_{b} \int_{\mathrm{reg}} \frac{d^{4} \ell}{(2 \pi)^{4}} \frac{1}{\ell^{2}-i \epsilon} \frac{\left(2 p_{a}-\ell\right) \cdot\left(2 p_{b}+\ell\right)}{\left(2 p_{a} \cdot \ell-\ell^{2}+i \epsilon\right)\left(2 p_{b} \cdot \ell+\ell^{2}-i \epsilon\right)} .
$$

So essentially we need to evaluate $K_{\mathrm{em}}^{\mathrm{reg}}$. For this we need to evaluate the integral: ${ }^{8}$

$$
\begin{aligned}
\mathcal{I}_{a b} & \equiv \int_{\mathrm{reg}} \frac{d^{4} \ell}{(2 \pi)^{4}} \frac{1}{\ell^{2}-i \epsilon} \frac{1}{p_{a} \cdot \ell+i \epsilon} \frac{1}{p_{b} \cdot \ell-i \epsilon} \\
& =-\frac{1}{E_{a} E_{b}} \int_{\operatorname{reg}} \frac{d^{3} \ell}{(2 \pi)^{3}} \int_{-\infty}^{\infty} \frac{d \ell^{0}}{2 \pi} \frac{1}{\left(\ell^{0}-|\vec{\ell}|+i \epsilon\right)\left(\ell^{0}+|\vec{\ell}|-i \epsilon\right)} \frac{1}{\ell^{0}-\vec{v}_{a} \cdot \vec{\ell}-i \epsilon} \frac{1}{\ell^{0}-\vec{v}_{b} \cdot \vec{\ell}+i \epsilon},
\end{aligned}
$$

where $E_{a}=p_{a}^{0}, E_{b}=p_{b}^{0}, \vec{v}_{a}=\vec{p}_{a} / E_{a}$ and $\vec{v}_{b}=\vec{p}_{b} / E_{b}$. In writing down the above equation we have assumed that $E_{a}$ and $E_{b}$ are positive, i.e. both lines represent incoming states. The integrand has simple poles at,

$$
\ell^{0}=(|\vec{\ell}|-i \epsilon),-(|\vec{\ell}|-i \epsilon),\left(\vec{v}_{a} \cdot \vec{\ell}+i \epsilon\right),\left(\vec{v}_{b} \cdot \vec{\ell}-i \epsilon\right) .
$$

So now if we close the contour in the lower half plane we have to take the pole contributions from $\ell^{0}=(|\vec{\ell}|-i \epsilon)$ and $\ell^{0}=(\vec{v} \cdot \vec{\ell}-i \epsilon)$. This gives

$$
\begin{aligned}
\mathcal{I}_{a b}= & \frac{i}{E_{a} E_{b}} \int_{\operatorname{reg}} \frac{d^{3} \vec{\ell}}{(2 \pi)^{3}} \frac{1}{2|\vec{\ell}|} \frac{1}{|\vec{\ell}|-\vec{v}_{a} \cdot \vec{\ell}} \frac{1}{|\vec{\ell}|-\vec{v}_{b} \cdot \vec{\ell}} \\
& +\frac{i}{E_{a} E_{b}} \int_{\text {reg }} \frac{d^{3} \vec{\ell}}{(2 \pi)^{3}} \frac{1}{\left(\vec{v}_{b} \cdot \vec{\ell}\right)^{2}-|\vec{\ell}|^{2}} \frac{1}{\left(\vec{v}_{b}-\vec{v}_{a}\right) \cdot \vec{\ell}-i \epsilon}
\end{aligned}
$$

Note that we have removed the $i \epsilon$ 's from the denominators that never vanish.

Let us first analyze the second term. Since the result should be Lorentz invariant, it should not depend on the chosen frame. For simplicity choose a frame in which $\vec{v}_{b}$ and $\vec{v}_{a}$ are along the positive $z$-axis with $\left|\vec{v}_{b}\right|>\left|\vec{v}_{a}\right|$. Denoting by $\theta$ the angle between $\vec{\ell}$ and the $z$-axis, we can express the second term in (5.24) as

$$
\mathcal{I}_{a b}^{\prime}=\frac{i}{E_{a} E_{b}(2 \pi)^{2}} \frac{1}{\left|\vec{v}_{a}-\vec{v}_{b}\right|} \int_{\mathrm{reg}} \frac{d|\vec{\ell}|}{|\vec{\ell}|} \int_{-1}^{1} d(\cos \theta) \frac{1}{\left|\vec{v}_{b}\right|^{2} \cos ^{2} \theta-1} \frac{1}{\cos \theta-i \epsilon} .
$$

\footnotetext{
${ }^{8}$ Since the $\ell^{\mu}$ integration runs over a limited range, one might wonder why we are choosing the $\ell^{0}$ integration range from $-\infty$ to $\infty$. To this end, note that once we have imposed the range restriction on $|\vec{\ell}|$, we can let the $\ell^{0}$ integral in (5.22) run over the entire real axis since the regions outside the allowed range do not generate any logarithmic contribution.
} 
Without the $i \epsilon$ piece of the last term the integral vanishes since the integrand is an odd function of $\cos \theta$. However the imaginary part of the last term makes the integral nonvanishing. Using $1 /(x-i \epsilon)=i \pi \delta(x)+P(1 / x)$ in the integral, and using the fact that the value of $|\vec{\ell}|$ for which our approximation of the integrand is valid ranges from $\omega$ to some finite energy, we get,

$$
\mathcal{I}_{a b}^{\prime} \simeq \frac{1}{4 \pi E_{a} E_{b}} \ln \omega^{-1} \frac{1}{\left|\vec{v}_{a}-\vec{v}_{b}\right|}=\frac{1}{4 \pi} \ln \omega^{-1} \frac{1}{\sqrt{\left(p_{a} \cdot p_{b}\right)^{2}-m_{a}^{2} m_{b}^{2}}}
$$

where in the intermediate stage we used $\left|\vec{p}_{a}\right|\left|\vec{p}_{b}\right|=\left|\vec{p}_{a} \cdot \vec{p}_{b}\right|$, since $\vec{p}_{a}$ and $\vec{p}_{b}$ are parallel.

If both the legs $a$ and $b$ are outgoing instead of ingoing, then $E_{a}$ and $E_{b}$ are negative and the signs of the $i \epsilon$ in the last two terms in (5.22) are reversed. But this can be brought back to the form given in (5.22) by making a change of variables $\ell^{\mu} \rightarrow-\ell^{\mu}$. Therefore the net result for the residue at $\ell^{0}=\vec{v}_{b} \cdot \vec{\ell}-i \epsilon$ will continue to be described by (5.26). Finally if one of the momenta is outgoing and the other is ingoing, then both the $i \epsilon$ 's in the last two terms of (5.22) come with the same sign. By changing variables from $\ell^{\mu}$ to $-\ell^{\mu}$ if necessary, we can ensure that both the poles are in the upper half plane and close the contour to the lower half plane. In this case there will be no analog of the contribution given in (5.26).

We now turn to the contribution from the first term on the right hand side of (5.24), which we will call $\mathcal{I}^{\prime \prime}{ }_{a b}$. We will again evaluate this integral in the frame in which $\vec{v}_{a}$ and $\vec{v}_{b}$ are parallel to the $z$-axis with $\left|\vec{v}_{b}\right|>\left|\vec{v}_{a}\right|$. We get

$$
\begin{aligned}
\mathcal{I}^{\prime \prime}{ }_{a b} & =\frac{i}{E_{a} E_{b}} \int_{\mathrm{reg}} \frac{d^{3} \vec{l}}{(2 \pi)^{3}} \frac{1}{2|\vec{l}|} \frac{1}{|\vec{l}|-\vec{v}_{a} \cdot \vec{l}} \frac{1}{|\vec{l}|-\vec{v}_{b} \cdot \vec{l}} \\
& =\frac{i}{8 \pi^{2} E_{a} E_{b}} \ln \omega^{-1} \int_{-1}^{1} d(\cos \theta) \frac{1}{v_{b}-v_{a}}\left[\frac{v_{b}}{1-v_{b} \cos \theta}-\frac{v_{a}}{1-v_{a} \cos \theta}\right] \\
& =\frac{i}{8 \pi^{2}} \ln \omega^{-1} \frac{1}{\left|\vec{p}_{b}\right| E_{a}-\left|\vec{p}_{a}\right| E_{b}} \ln \left[\frac{\left(E_{a}-\left|\vec{p}_{a}\right|\right)\left(E_{b}+\left|\vec{p}_{b}\right|\right)}{\left(E_{a}+\left|\vec{p}_{a}\right|\right)\left(E_{b}-\left|\vec{p}_{b}\right|\right)}\right] \\
& =-\frac{i}{8 \pi^{2}} \ln \omega^{-1} \frac{1}{\sqrt{\left(p_{a} \cdot p_{b}\right)^{2}-p_{a}^{2} p_{b}^{2}}} \ln \left[\frac{p_{a} \cdot p_{b}+\sqrt{\left(p_{a} \cdot p_{b}\right)^{2}-p_{a}^{2} p_{b}^{2}}}{p_{a} \cdot p_{b}-\sqrt{\left(p_{a} \cdot p_{b}\right)^{2}-p_{a}^{2} p_{b}^{2}}}\right] .
\end{aligned}
$$

It is easy to check that the form of the contribution remains unchanged even when both legs are outgoing or one leg is incoming and the other leg is outgoing.

Combining these results we get

$$
K_{\mathrm{em}}^{\mathrm{reg}}=\frac{i}{2} \sum_{\substack{a, b \\ b \neq a}} q_{a} q_{b} \frac{1}{4 \pi} \ln \omega^{-1} \frac{p_{a} \cdot p_{b}}{\sqrt{\left(p_{a} \cdot p_{b}\right)^{2}-p_{a}^{2} p_{b}^{2}}}\left\{\delta_{\eta_{a} \eta_{b}, 1}-\frac{i}{2 \pi} \ln \left(\frac{p_{a} \cdot p_{b}+\sqrt{\left(p_{a} \cdot p_{b}\right)^{2}-p_{a}^{2} p_{b}^{2}}}{p_{a} \cdot p_{b}-\sqrt{\left(p_{a} \cdot p_{b}\right)^{2}-p_{a}^{2} p_{b}^{2}}}\right)\right\}
$$

Using (5.20) we can now write down the expression for the logarithmic term in the sub- 
leading soft factor $S_{\mathrm{em}}^{(1)}$

$$
\begin{aligned}
& -\frac{i}{4 \pi} \ln \omega^{-1} \sum_{a=1}^{n} \sum_{\substack{b \neq a \\
\eta_{a} \eta_{b}=1}} q_{a}^{2} q_{b} \frac{\epsilon_{\mu} k_{\rho}}{p_{a} \cdot k} \frac{m_{a}^{2} m_{b}^{2}\left[p_{a}^{\mu} p_{b}^{\rho}-p_{b}^{\mu} p_{a}^{\rho}\right]}{\left[\left(p_{a} \cdot p_{b}\right)^{2}-m_{a}^{2} m_{b}^{2}\right]^{3 / 2}} \\
& -\frac{1}{8 \pi^{2}} \ln \omega^{-1} \sum_{\substack{a, b \\
b \neq a}} q_{a}^{2} q_{b} \ln \left[\frac{p_{a} \cdot p_{b}+\sqrt{\left(p_{a} \cdot p_{b}\right)^{2}-p_{a}^{2} p_{b}^{2}}}{p_{a} \cdot p_{b}-\sqrt{\left(p_{a} \cdot p_{b}\right)^{2}-p_{a}^{2} p_{b}^{2}}}\right] \frac{p_{a}^{2} p_{b}^{2}}{\left\{\left(p_{a} \cdot p_{b}\right)^{2}-p_{a}^{2} p_{b}^{2}\right\}^{3 / 2}}\left\{-\epsilon \cdot p_{b}+\frac{\epsilon \cdot p_{a}}{k \cdot p_{a}} k \cdot p_{b}\right\} \\
& +\frac{1}{4 \pi^{2}} \ln \omega^{-1} \sum_{\substack{a, b \\
b \neq a}} q_{a}^{2} q_{b} \frac{p_{a} \cdot p_{b}}{\left(p_{a} \cdot p_{b}\right)^{2}-p_{a}^{2} p_{b}^{2}}\left\{-\epsilon \cdot p_{b}+\frac{\epsilon \cdot p_{a}}{k \cdot p_{a}} k \cdot p_{b}\right\} .
\end{aligned}
$$

The term in the first line agrees with the classical expression for $S_{\mathrm{em}}^{(1)}$ given by the second term of (3.16). The rest of the contribution is extra.

We have also checked that (5.29) holds if instead of scalars we have interacting fermions. This confirms that the logarithmic correction to the soft factor is independent of the spin of the particle.

We end this section by making some observation on the results derived above:

1. Suppose we assume the validity of the naive version of the subleading soft photon theorem: ${ }^{9}$

$$
\Gamma^{(n, 1)}=\left\{S_{\mathrm{em}}^{(0)}+\widehat{S}_{\mathrm{em}}^{(1)}\right\} \Gamma^{(n)},
$$

where the 'hat' on $S^{(1)}$ denotes that we are using the differential operator form that arises in the quantum theory:

$$
S_{\mathrm{em}}^{(0)}=\sum_{a} q_{a} \frac{\varepsilon \cdot p_{a}}{p_{a} \cdot k}, \quad \widehat{S}_{\mathrm{em}}^{(1)}=\sum_{a} q_{a} \frac{\varepsilon_{\mu} k_{\nu}}{p_{a} \cdot k}\left\{p_{a}^{\mu} \frac{\partial}{\partial p_{a \nu}}-p_{a}^{\nu} \frac{\partial}{\partial p_{a \mu}}\right\} .
$$

Then using (5.7) and the fact that $\Gamma_{\mathrm{G}}^{(n)}$ vanishes at one loop order, we get

$$
\Gamma_{\text {tree }}^{(n, 1)}+\Gamma_{\text {self }}^{(n, 1)}+\Gamma_{\mathrm{G}}^{(n, 1)}=S_{\mathrm{em}}^{(0)} \Gamma_{\text {tree }}^{(n)}+\left\{\widehat{S}_{\mathrm{em}}^{(1)} K_{\mathrm{em}}\right\} \Gamma_{\text {tree }}^{(n)}+\widehat{S}_{\mathrm{em}}^{(1)} \Gamma_{\text {tree }}^{(n)} .
$$

Using $\Gamma_{\text {tree }}^{(n)}=i \lambda$, using (5.10) to replace the left hand side, and throwing away terms like $\widehat{S}_{\mathrm{em}}^{(1)} \Gamma_{\text {tree }}^{(n)}$ which vanishes, we get

$$
S_{\mathrm{em}}^{(1)}=\widehat{S}_{\mathrm{em}}^{(1)} K_{\mathrm{em}} .
$$

In the definition of $K_{\mathrm{em}}$ the integration over loop momentum runs over all range and we have an infrared divergence from the region of small $\ell$. However if we make an ad hoc restriction that the loop momentum integral will run in the range much larger than the energy $\omega$ of the external soft photon, then $K_{\mathrm{em}}$ reduces to $K_{\mathrm{em}}^{\mathrm{reg}}$ defined in (5.21) and we recover the correct logarithmic terms in $S_{\mathrm{em}}^{(1)}$ as given in (5.20). This suggests an ad hoc rule for computing the logarithmic terms in the soft expansion

\footnotetext{
${ }^{9}$ Since the presence of the logarithmic term makes the finite part ambiguous, we consider only the logarithmic terms in the subleading factor.
} 
in quantum theory - begin with the usual soft expansion and explicitly evaluate the action of the differential operator on the amplitude, restricting the region of loop momentum integration to lie in a range larger than the soft momenta but smaller than the momenta of the finite energy particles. With hindsight, this prescription can be justified by noting that the general arguments of [81, 83], that assumes existence of 1PI effective action with no powers of soft momenta coming from the vertices, breaks down for the contribution where the loop momentum is smaller than the external soft momenta. On the other hand we do not expect any large contribution from the region of integration where the loop momentum is of the order of the external momenta or larger.

This argument also suggests that although we have carried out the explicit calculation only at one loop order, the result may be valid to all orders in perturbation theory, since $K_{\mathrm{em}}$ is known to be valid to all orders in perturbation theory [99].

2. The second observation concerns the relation between the classical and the quantum results. As already noted, compared to the classical result that agrees with the first line of (5.29), the quantum result found here has an extra term given in the second and third line of (5.29). If however we replace in (5.22) the Feynman propagator for the photon by the retarded propagator, we get only the contribution from the first line of (5.29), since the contribution from the pole at $\ell^{2}=0$ can then be avoided by appropriate choice of contour. Therefore at least for the soft photon theorem in quantum electrodynamics, the rule for relating the quantum and the classical result seems to be to replace the Feynman propagator of the photon in the loop in the quantum result by retarded propagator.

We shall now write down the results for the other cases and test if the generalization of observation 1 works. We shall also explore if the results satisfy the generalization of observation 2 .

\section{$6 \quad$ Soft graviton theorem in gravitational scattering}

We now turn to the analysis of the soft graviton theorem in the scattering of scalar particles, interacting via gravity, to one loop order. The action is taken to be

$$
\int d^{4} x \sqrt{-\operatorname{det} g}\left[\frac{1}{16 \pi G} R-\sum_{a=1}^{n}\left\{g^{\mu \nu} \partial_{\mu} \phi_{a}^{*} \partial_{\nu} \phi_{a}+m_{a}^{2} \phi_{a}^{*} \phi_{a}\right\}+\lambda \phi_{1} \cdots \phi_{n}+\lambda \phi_{1}^{*} \cdots \phi_{n}^{*}\right] .
$$

Even though in this case we could take the scalar fields to be real, we have kept them complex in order to extend the analysis to the case where the scalars have both electromagnetic and gravitational interaction. As in section 5, we shall postulate a relation of the form

$$
\Gamma^{(n, 1)}=\left\{S_{\mathrm{gr}}^{(0)}+S_{\mathrm{gr}}^{(1)}\right\} \Gamma^{(n)},
$$

and try to determine the logarithmic terms in $S_{\mathrm{gr}}^{(1)}$ by comparing the two sides up to one loop order. 
We shall carry out our computation in the de Donder gauge in which the propagator of a graviton of momentum $\ell$ is given by:

$$
-\frac{i}{\ell^{2}-i \epsilon} \frac{1}{2}\left(\eta^{\mu \rho} \eta^{\nu \sigma}+\eta^{\mu \sigma} \eta^{\nu \rho}-\eta^{\mu \nu} \eta^{\rho \sigma}\right)
$$

For our analysis we also need the vertices involving the graviton. The scalar-scalar-graviton vertex, with the scalars carrying ingoing momenta $p_{1}, p_{2}$ and the graviton carrying ingoing momentum $-p_{1}-p_{2}$ and Lorentz index $(\mu \nu)$, is given by

$$
-i \kappa\left[p_{1 \mu} p_{2 \nu}+p_{1 \nu} p_{2 \mu}-\eta_{\mu \nu}\left(p_{1} \cdot p_{2}-m^{2}\right)\right]
$$

where $\kappa=\sqrt{8 \pi G}=1$ in our convention. The vertex involving two scalars carrying ingoing momenta $p_{1}, p_{2}$, and two gravitons carrying ingoing momenta $k_{1}, k_{2}$ and Lorentz indices $(\alpha \beta)$ and $(\mu \nu)$ is given by ${ }^{10}$

$$
\begin{aligned}
2 i \kappa^{2}[- & \eta_{\alpha \mu} \eta_{\beta \nu} p_{1} \cdot p_{2}+\frac{1}{2} \eta_{\alpha \beta} \eta_{\mu \nu} p_{1} \cdot p_{2}-\eta_{\alpha \beta} p_{1 \mu} p_{2 \nu}-\eta_{\mu \nu} p_{1 \alpha} p_{2 \beta} \\
& \left.+2 \eta_{\alpha \mu}\left\{p_{1 \beta} p_{2 \nu}+p_{2 \beta} p_{1 \nu}\right\}+m^{2}\left(\eta_{\mu \alpha} \eta_{\nu \beta}-\frac{1}{2} \eta_{\mu \nu} \eta_{\alpha \beta}\right)\right] .
\end{aligned}
$$

If we label the ingoing graviton momenta by $k_{1}, k_{2}$ and $k_{3}=-k_{1}-k_{2}$ and the Lorentz indices carried by them by $(\mu \alpha),(\nu \beta)$ and $(\sigma \gamma)$ respectively, then the 3 -graviton vertex takes the form:

$$
\begin{aligned}
i \kappa[ & \left(k_{1} . k_{2} \eta_{\mu \alpha} \eta_{\nu \sigma} \eta_{\beta \gamma}+k_{2} . k_{1} \eta_{\nu \beta} \eta_{\mu \sigma} \eta_{\alpha \gamma}+k_{1} . k_{3} \eta_{\mu \alpha} \eta_{\nu \sigma} \eta_{\beta \gamma}\right. \\
& \left.+k_{3} \cdot k_{1} \eta_{\sigma \gamma} \eta_{\mu \nu} \eta_{\alpha \beta}+k_{2} . k_{3} \eta_{\nu \beta} \eta_{\mu \sigma} \eta_{\alpha \gamma}+k_{3} . k_{2} \eta_{\sigma \gamma} \eta_{\mu \nu} \eta_{\alpha \beta}\right) \\
& -2\left(k_{1 \sigma} k_{2 \gamma} \eta_{\mu \nu} \eta_{\alpha \beta}+k_{2 \mu} k_{3 \alpha} \eta_{\nu \sigma} \eta_{\beta \gamma}+k_{3 \nu} k_{1 \beta} \eta_{\mu \sigma} \eta_{\alpha \gamma}\right) \\
& -4\left(k_{1} \cdot k_{2}+k_{2} . k_{3}+k_{3} . k_{1}\right) \eta_{\alpha \nu} \eta_{\beta \sigma} \eta_{\gamma \mu} \\
& +\left(k_{1} \cdot k_{2} \eta_{\mu \nu} \eta_{\alpha \beta} \eta_{\sigma \gamma}+k_{2} . k_{3} \eta_{\nu \sigma} \eta_{\beta \gamma} \eta_{\mu \alpha}+k_{3} . k_{1} \eta_{\mu \sigma} \eta_{\alpha \gamma} \eta_{\nu \beta}\right) \\
& +2\left(k_{1 \sigma} k_{2 \mu} \eta_{\alpha \nu} \eta_{\beta \gamma}+k_{2 \mu} k_{3 \nu} \eta_{\sigma \alpha} \eta_{\gamma \beta}+k_{3 \nu} k_{1 \sigma} \eta_{\mu \beta} \eta_{\alpha \gamma}\right. \\
& \left.+k_{2 \sigma} k_{1 \nu} \eta_{\mu \beta} \eta_{\alpha \gamma}+k_{3 \mu} k_{2 \sigma} \eta_{\nu \gamma} \eta_{\beta \alpha}+k_{1 \nu} k_{3 \mu} \eta_{\sigma \beta} \eta_{\gamma \alpha}\right) \\
& \left.-\frac{1}{2}\left(k_{1} \cdot k_{2}+k_{2} . k_{3}+k_{3} \cdot k_{1}\right) \eta_{\mu \alpha} \eta_{\nu \beta} \eta_{\sigma \gamma}\right] .
\end{aligned}
$$

In (6.5) and (6.6) it is understood that the vertices need to be symmetrized under the exchange of the pair of Lorentz indices carried by each external graviton, e.g. $\mu \leftrightarrow \nu$ and $\alpha \leftrightarrow \beta$ in (6.5) and $\mu \leftrightarrow \alpha, \nu \leftrightarrow \beta$ and $\sigma \leftrightarrow \gamma$ in (6.6). Even though (6.6) has a complicated form, we shall need the form of the vertex when one of the external momenta (say $k_{3}$ ) is small compared to the others. In this limit it simplifies.

The vertex where a graviton carrying Lorentz index $(\mu \nu)$ attaches to $n$ scalar fields is given by:

$$
i \kappa \lambda \eta_{\mu \nu}
$$

\footnotetext{
${ }^{10}$ In writing this and other vertices we already include the symmetry factor related to exchange of identical particles. Therefore if we were to use this vertex to compute tree level two graviton, two scalar amplitude, no further symmetry factor is necessary.
} 

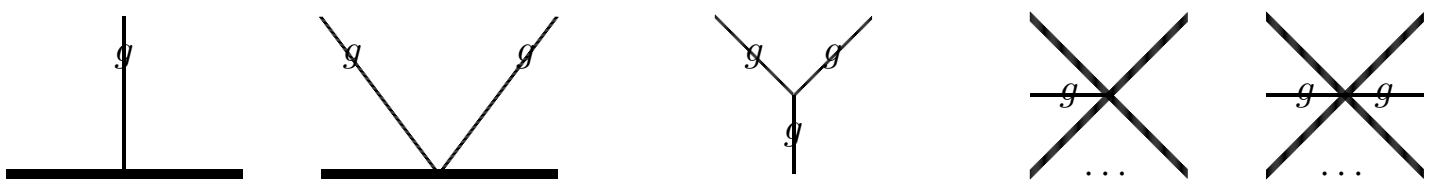

Figure 6. This diagram shows various vertices induced from the action (6.1) that are needed for our computation. Here the thinner lines carrying the symbol $g$ denote gravitons and the thicker lines denote scalars.

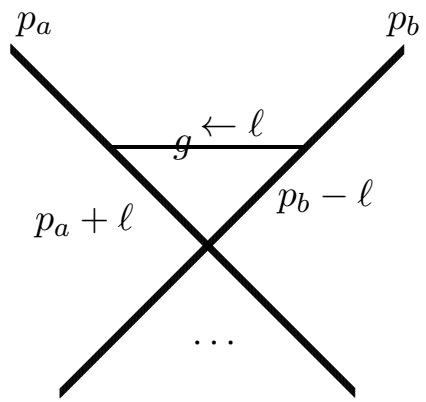

Figure 7. Diagram contributing to $\Gamma^{(n)}$.

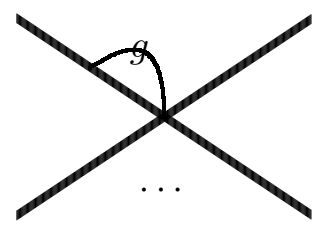

Figure 8. Another diagram contributing to $\Gamma^{(n)}$. We can also have a diagram where both ends of the internal graviton are attached to the $n$-scalar vertex, but this vanishes in dimensional regularization and so we have not displayed them.

The vertex where two gravitons carrying Lorentz index $(\mu \nu)$ and $(\rho \sigma)$ attach to $n$ scalar fields is given by:

$$
-i \kappa^{2} \lambda\left(\eta_{\mu \nu} \eta_{\rho \sigma}-\eta_{\mu \rho} \eta_{\nu \sigma}-\eta_{\mu \sigma} \eta_{\nu \rho}\right)
$$

We also need the vertex containing two scalars and three gravitons for evaluating the fifth diagram of figure 10. However even without knowing the form of this vertex one can see that this diagram does not generate contributions proportional to $\ln \omega^{-1}$. Therefore we have not written down the expression for this vertex.

We can use these vertices to compute one loop contribution to the $n$ scalar amplitude $\Gamma^{(n)}$ and $n$-scalar and one soft graviton amplitude $\Gamma^{(n, 1)}$. At one loop order $\Gamma^{(n)}$ receives contribution from diagrams shown in figure 7 that are analogous to figure 3 with the internal photon replaced by a graviton. There are also some additional diagrams shown in figure 8 .

The relevant diagrams for $\Gamma^{(n, 1)}$ include the analogs figures 1 and 2 with all photons replaced by gravitons. This have been shown in figures 9 and 10. However there are also some extra diagrams that we shall list below: 


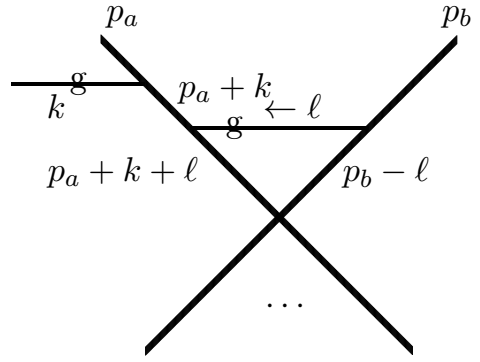

(a)

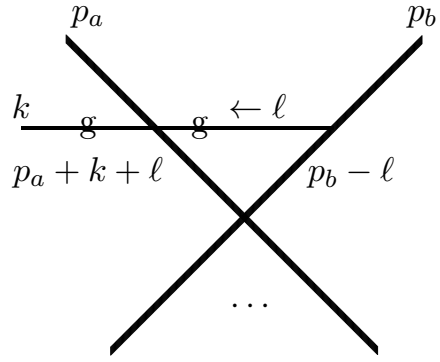

(b)

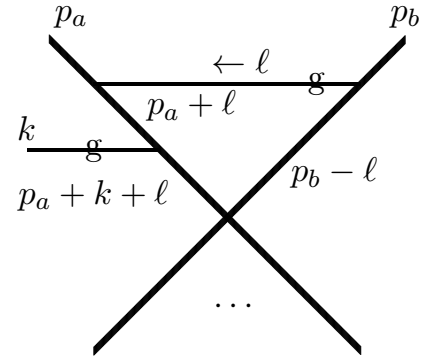

(c)

Figure 9. One loop contribution to $\Gamma^{(n, 1)}$ involving internal graviton line connecting two different legs. The thicker lines represent scalar particles and the thinner lines represent gravitons.
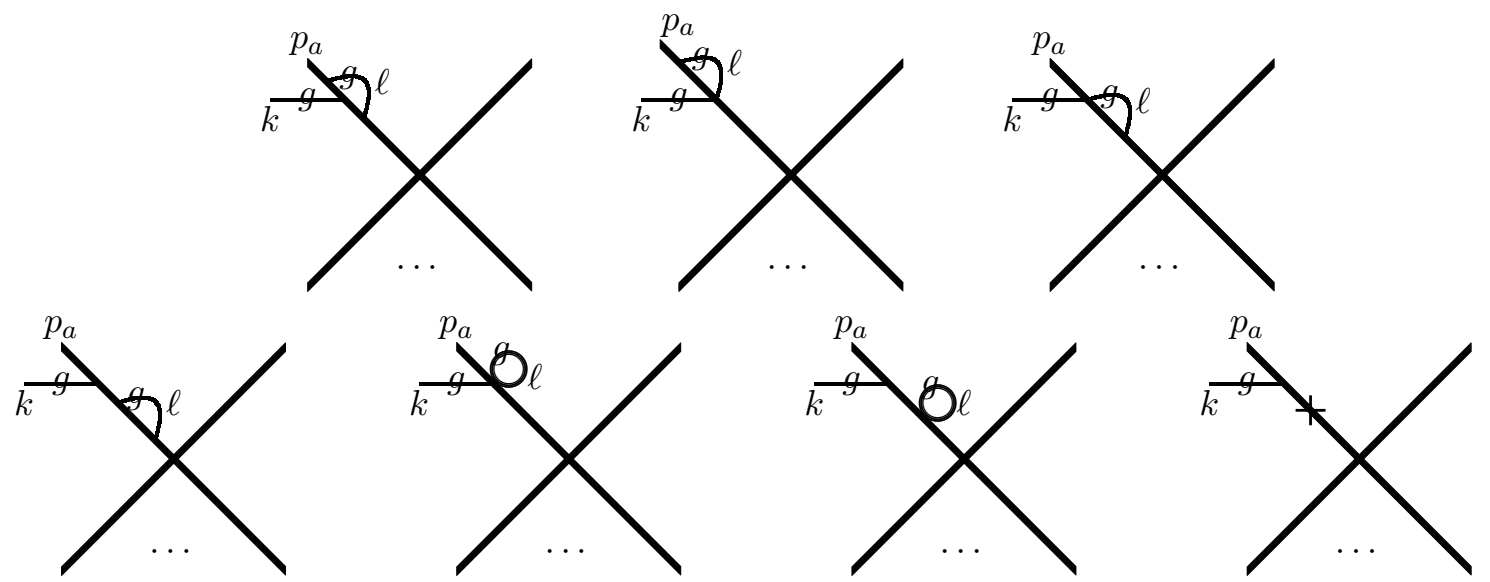

Figure 10. One loop contribution to $\Gamma^{(n, 1)}$ involving internal graviton line connecting two different points on the same leg.

1. There are diagrams where the external graviton couples to the internal graviton via the cubic coupling (6.6). Examples of these are shown in figure 11.

2. There are diagrams where one end of the internal graviton attaches to the $n$-scalar vertex via the coupling (6.7). These have been shown in figures 12 .

3. There are diagrams where the external graviton attaches to the scalar $n$-point vertex via the coupling (6.7) or (6.8). These have been shown in figure 13. The first diagram can be made to vanish by taking the external graviton polarization to be traceless: $\varepsilon_{\rho}^{\rho}=0$. The second diagram has no logarithmic terms. Therefore we shall ignore these diagrams in subsequent discussions.

4. There are diagrams of the type shown in figure 14 where two ends of the internal graviton attach to the $n$-scalar vertex. In dimensional regularization these diagrams vanish. Therefore we shall ignore these diagrams in our analysis.

Our analysis of these diagrams will proceed as in section 5, but there will be some important differences that we shall point out below. For an internal graviton of momentum 

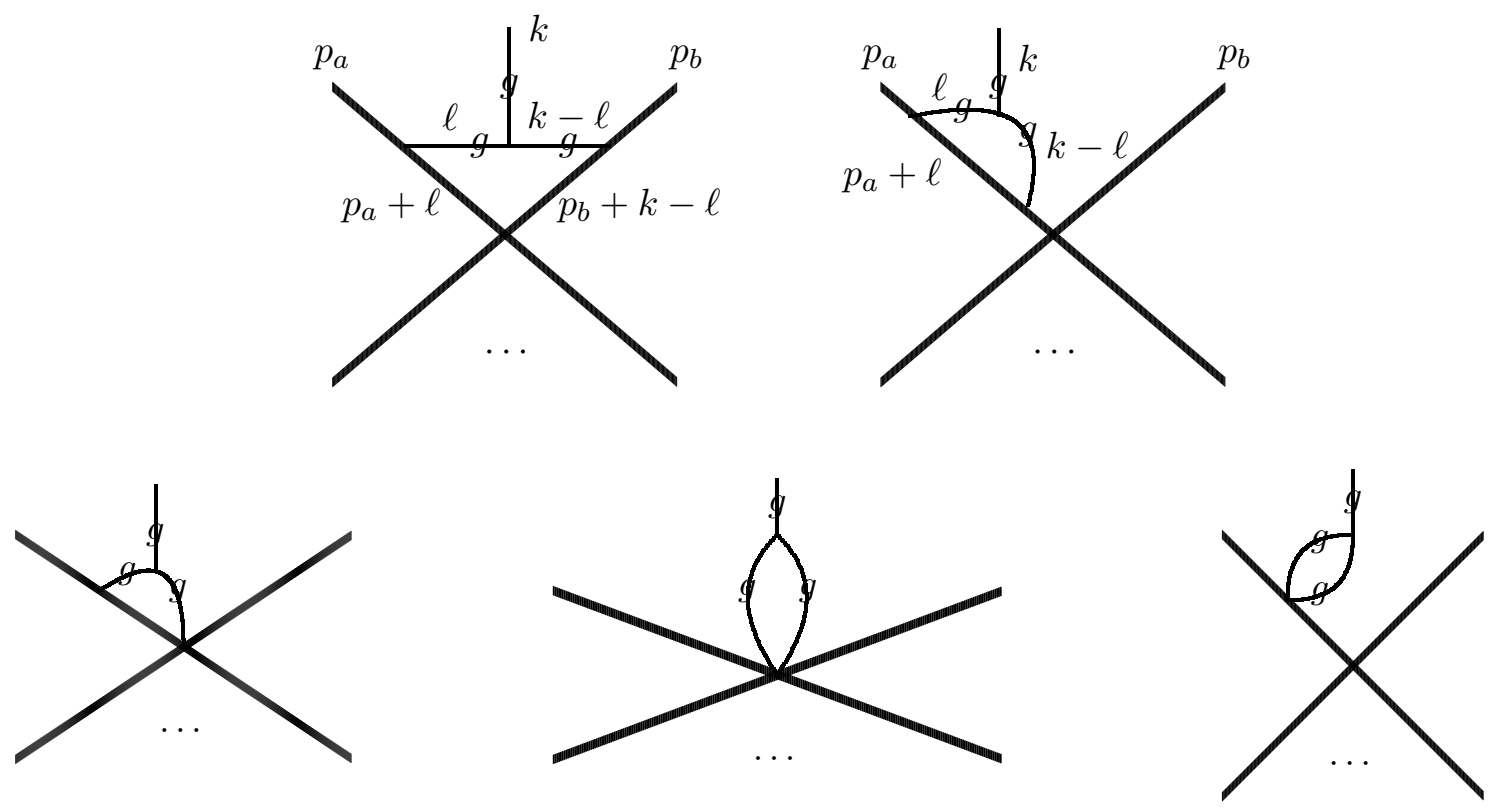

Figure 11. Diagrams involving 3-graviton vertex.
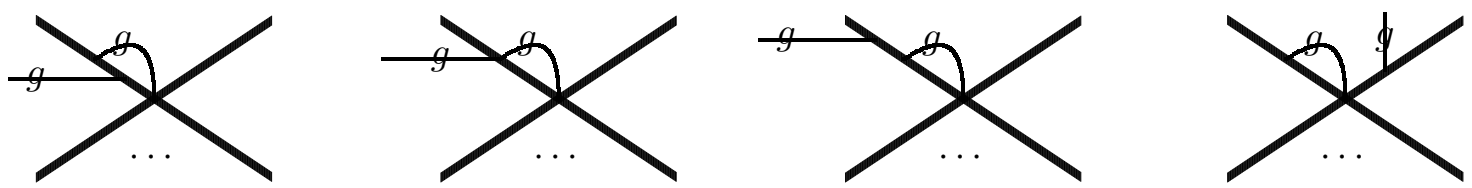

Figure 12. Diagrams where the internal graviton attaches to the $n$-point vertex.
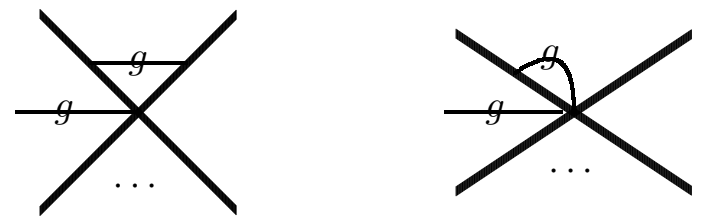

Figure 13. Diagrams where the external graviton attaches to the $n$-point vertex. The first diagram vanishes if we take the external graviton polarization to be traceless. The second diagram has no logarithmic terms.
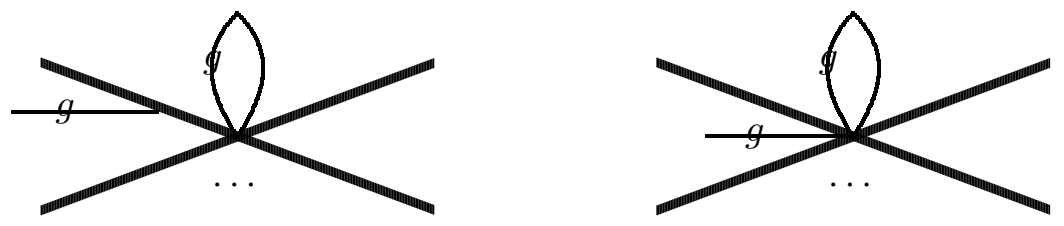

Figure 14. Diagrams where both ends of the internal graviton attach to the $n$-point vertex. In dimensional regularization these diagrams vanish. Even if we use momentum cut-off, these diagrams cannot have any contribution proportional to $\ln \omega^{-1}$ since the soft momentum $k$ does not flow through any loop. 
$\ell$, whose two ends are attached to two scalar lines $a$ and $b$ with $\ell$ flowing from the leg $b$ towards the leg $a$, as in figures 7, 9, the analog of Grammer-Yennie decomposition of the graviton propagator will be taken to be

$$
\begin{aligned}
& G_{(a b)}^{\mu \nu, \rho \sigma}\left(\ell, p_{a}, p_{b}\right)=\left(\eta^{\mu \rho} \eta^{\nu \sigma}+\eta^{\mu \sigma} \eta^{\nu \rho}-\eta^{\mu \nu} \eta^{\rho \sigma}\right)-K_{(a b)}^{\mu \nu, \rho \sigma}\left(\ell, p_{a}, p_{b}\right), \\
& K_{(a b)}^{\mu \nu, \rho \sigma}\left(\ell, p_{a}, p_{b}\right)=\mathcal{C}\left(\ell, p_{a}, p_{b}\right)\left[\left(p_{a}+\ell\right)^{\mu} \ell^{\nu}+\left(p_{a}+\ell\right)^{\nu} \ell^{\mu}\right]\left[\left(p_{b}-\ell\right)^{\rho} \ell^{\sigma}+\left(p_{b}-\ell\right)^{\sigma} \ell^{\rho}\right],
\end{aligned}
$$

where

$$
\begin{aligned}
\mathcal{C}\left(\ell, p_{a}, p_{b}\right)= & \frac{(-1)}{\left\{p_{a} \cdot\left(p_{a}+\ell\right)-i \epsilon\right\}\left\{p_{b} \cdot\left(p_{b}-\ell\right)-i \epsilon\right\}\left\{\ell \cdot\left(\ell+2 p_{a}\right)-i \epsilon\right\}\left\{\ell \cdot\left(\ell-2 p_{b}\right)-i \epsilon\right\}} \\
& {\left[2\left(p_{a} \cdot p_{b}\right)^{2}-p_{a}^{2} p_{b}^{2}-\ell^{2}\left(p_{a} \cdot p_{b}\right)-2\left(p_{a} \cdot p_{b}\right)\left(p_{a} \cdot \ell\right)+2\left(p_{a} \cdot p_{b}\right)\left(p_{b} \cdot \ell\right)\right] . }
\end{aligned}
$$

If one end of an internal graviton is attached to the $n$-scalar vertex and the other end is attached to the $a$ 'th scalar leg as in figures 8,12 , with $\ell$ flowing from the vertex towards the $a$ 'th leg, we express the propagator as:

$$
-\frac{i}{\ell^{2}-i \epsilon} \frac{1}{2}\left\{G_{(a)}^{\mu \nu, \rho \sigma}\left(\ell, p_{a}\right)+K_{(a)}^{\mu \nu, \rho \sigma}\left(\ell, p_{a}\right)\right\},
$$

where

$$
\begin{gathered}
G_{(a)}^{\mu \nu, \rho \sigma}\left(\ell, p_{a}\right)=\left(\eta^{\mu \rho} \eta^{\nu \sigma}+\eta^{\mu \sigma} \eta^{\nu \rho}-\eta^{\mu \nu} \eta^{\rho \sigma}\right)-K_{(a)}^{\mu \nu, \rho \sigma}\left(\ell, p_{a}\right) \\
K_{(a)}^{\mu \nu, \rho \sigma}\left(\ell, p_{a}\right)=\widetilde{\mathcal{C}}\left(\ell, p_{a}\right)\left[\left(p_{a}+\ell\right)^{\mu} \ell^{\nu}+\left(p_{a}+\ell\right)^{\nu} \ell^{\mu}\right] \eta^{\rho \sigma},
\end{gathered}
$$

and

$$
\widetilde{\mathcal{C}}\left(\ell, p_{a}\right)=-\frac{2 p_{a} \cdot\left(p_{a}-\ell\right)}{\left\{p_{a} \cdot\left(p_{a}+\ell\right)-i \epsilon\right\}\left\{\ell \cdot\left(\ell+2 p_{a}\right)-i \epsilon\right\}} .
$$

For internal gravitons whose one end is attached to a 3 -graviton vertex instead of a scalar, as in figure 11, we do not carry out any Grammer-Yennie decomposition.

The decomposition into $\mathrm{G}$ and $\mathrm{K}$-gravitons is not arbitrary but has been chosen to ensure two properties:

1. The K-graviton polarization, being proportional to $\ell$, is pure gauge and allows us to sum over K-graviton insertions using Ward identities. The relevant Ward identities have been shown in figure 15 , with the quantity $A(p, k, \ell, \xi, \zeta)$ is given by

$$
\begin{aligned}
A(p, k, \ell, \xi, \zeta)= & 2 i \xi \cdot p \zeta^{\mu \nu}\left[2\left(2 p_{\mu}+\ell_{\mu}\right) k_{\nu}+2 k_{\mu} k_{\nu}-\eta_{\mu \nu}\left\{k \cdot(2 p+\ell)+k^{2}\right\}\right] \\
& +2 i \xi \cdot(k+\ell) \zeta^{\mu \nu}\left[-2 p_{\mu}(p+\ell)_{\nu}+\eta_{\mu \nu}\left\{p \cdot(p+\ell)+m^{2}\right\}\right] \\
& +2 i\left(\xi^{\alpha} k^{\beta}+\xi^{\beta} k^{\alpha}\right) \zeta^{\mu \nu}\left[\eta_{\alpha \mu} \eta_{\beta \nu} p \cdot(p+k+\ell)-\frac{1}{2} \eta_{\alpha \beta} \eta_{\mu \nu} p \cdot(p+k+\ell)\right. \\
& +\eta_{\alpha \beta} p_{\mu}(p+k+\ell)_{\nu}+\eta_{\mu \nu} p_{\alpha}(p+k+\ell)_{\beta}-2 \eta_{\alpha \mu} p_{\beta}(p+k+\ell)_{\nu} \\
& \left.-2 \eta_{\alpha \mu} p_{\nu}(p+k+\ell)_{\beta}+m^{2}\left(\eta_{\mu \alpha} \eta_{\nu \beta}-\frac{1}{2} \eta_{\mu \nu} \eta_{\alpha \beta}\right)\right] .
\end{aligned}
$$

Due to this additional term, the sum over K-gravitons will leave behind some residual terms that will be discussed below. 

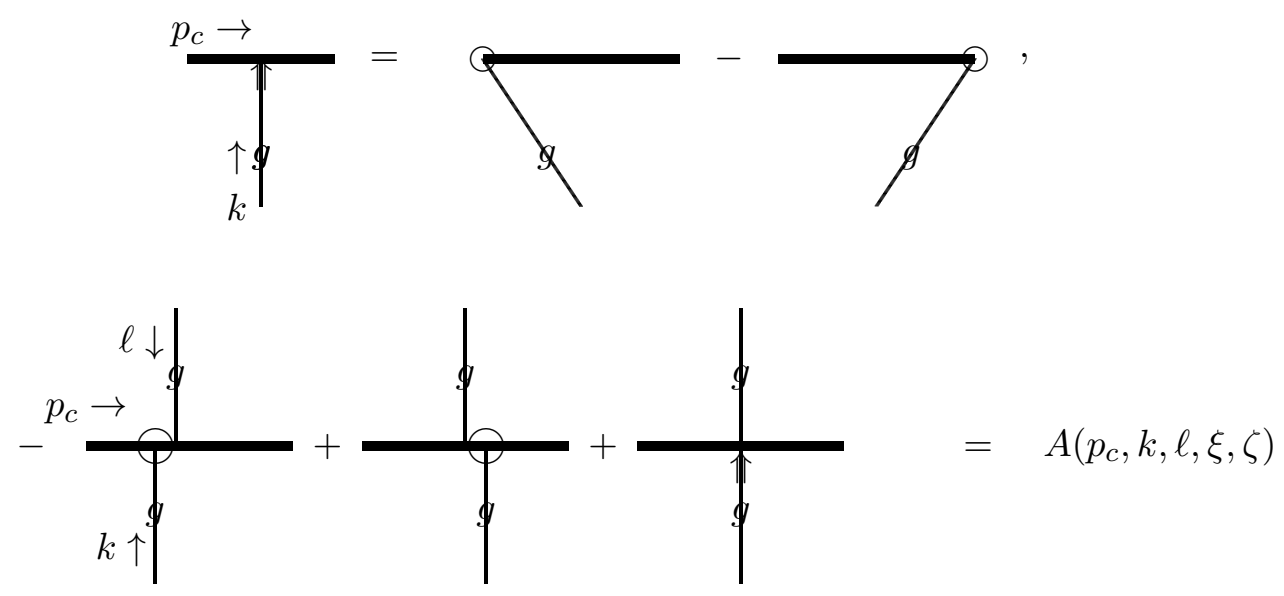

Figure 15. Analog of figure 4 for gravity. The arrow on the graviton line represents that the polarization of the graviton carrying momentum $k$ is taken to be equal to $\xi_{\mu} k_{\nu}+\xi_{\nu} k_{\mu}$. The polarization of the graviton carrying momentum $k$ is taken to be $\zeta_{\rho \sigma}$. In the first diagram the circle on the left denotes a vertex $-2 \xi .\left(p_{c}+k\right)$ while the circle on the right denotes a vertex $-2 \xi . p_{c}$. $A\left(p_{c}, k, \ell, \xi, \zeta\right)$ appearing on the right hand side of the second diagram is given in eq. (6.15).

2. In any one loop diagram contributing to the amplitude $\Gamma^{(n)}$ without external soft graviton, the result vanishes if we replace the internal graviton by G-graviton.

With this convention the K-graviton contribution to figure 7 for gravity can be computed as in section 5, leading to a contribution of the form $i \lambda K_{\mathrm{gr}}$ to $\Gamma^{(n)}$, where $K_{\mathrm{gr}}$ is the gravitational counterpart of $K_{\mathrm{em}}$. The relevant part of the expression for $K_{\mathrm{gr}}$ will be described later. The K-graviton contribution to figure 8 can be carried out similarly, leading to an expression of the form $i \lambda \widetilde{K}_{\mathrm{gr}}$. $\widetilde{K}_{\mathrm{gr}}$ has no infrared divergence and we shall not write down its expression explicitly although it is straightforward to do so. The G-graviton contributions to figures 7 and 8 vanish by construction. Therefore the net contribution to $\Gamma^{(n)}$ to one loop order may be written as $i \lambda \exp \left[K_{\mathrm{gr}}+\widetilde{K}_{\mathrm{gr}}\right]$.

The K-graviton contributions to figures 9 and 12 may be evaluated similarly, with the factorized term giving $i \lambda S_{\mathrm{gr}}^{(0)} \exp \left[K_{\mathrm{gr}}+\widetilde{K}_{\mathrm{gr}}\right]$. There are however some left-over terms arising as follows:

1. As shown in figure 15 , in the sum over K-graviton insertions in $\Gamma^{(n, 1)}$ there is a residual contribution $A$ that comes from lack of complete cancellation among terms where a K-graviton is inserted to the two sides of a scalar-scalar-graviton vertex and into the scalar-scalar-graviton vertex.

2. As explained in the caption of figure 15 , the circled vertices are momentum dependent. Therefore the two circled vertices shown in figure 16 are not the same, one carries a factor of $\xi \cdot p_{a}$ while the other carries a factor of $\xi \cdot\left(p_{a}+k\right)$. The left hand figure is relevant for $\Gamma^{(n)}$ while the right-hand figure is relevant for $\Gamma^{(n, 1)}$. Therefore, even after factoring out $\exp \left[K_{\mathrm{gr}}+\widetilde{K}_{\mathrm{em}}\right]$ factor multiplying $\Gamma^{(n)}$, we are left with an additional contribution to $\Gamma^{(n, 1)}$ from sum over K-gravitons that must be accounted for. 



Figure 16. Figure illustrating the difference in the factorized K-graviton contribution to $\Gamma^{(n)}$ and $\Gamma^{(n+1)}$.

We shall denote the sum of these two types of residual contributions as $\Gamma_{\text {residual }}^{(n, 1)}$. The Ggraviton contributions to figures 9 and 12 will be denoted by $\Gamma_{G}^{(n, 1)}$ and the net contribution from figure 10 will be called $\Gamma_{\text {self }}^{(n, 1)}$. Finally the contribution to the diagrams in figure 11

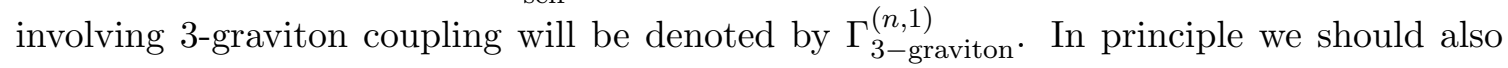
include the contributions from figure 13 and figure 14, but we ignore them since they do not generate logarithmic terms. In this case the analog of (5.11) takes the form:

$$
\Gamma_{\text {self }}^{(n, 1)}+\Gamma_{\mathrm{G}}^{(n, 1)}+\Gamma_{3 \text {-graviton }}^{(n, 1)}+\Gamma_{\text {residual }}^{(n, 1)}=i \lambda S_{\mathrm{gr}}^{(1)}
$$

We shall now briefly describe how we evaluate these contributions and then give the final result. First let us consider $\Gamma_{\text {residual }}^{(n, 1)}$. This receives contribution from figure 9 and figure 12. As explained above, there are two kinds of terms: one due to the right hand side of the second figure of figure 15, and the other due to the momentum dependence of the circled vertices in figure 15. It turns out that the residual part of the K-graviton contribution from figure 12 does not have any logarithmic term. On the other hand the residual part of the K-graviton contribution from figure 9 receives logarithmic contribution only from the region where the loop momentum is large compared to $\omega$. The result takes the form:

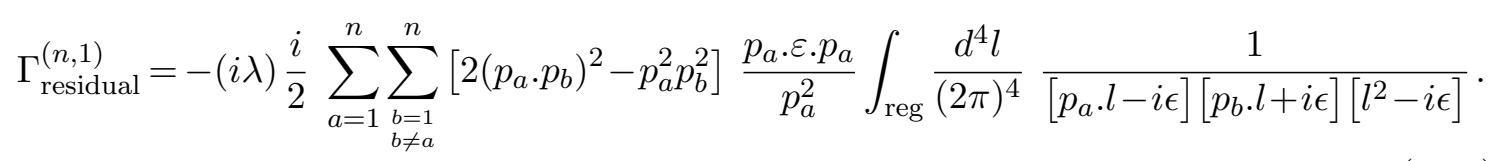

This contribution may be evaluated following a procedure similar to the one used in section 5 .

Contribution to $\Gamma_{3 \text {-graviton }}^{(n, 1)}$ arises from the five diagrams in figure 11, but only the first two give terms proportional to $\ln \omega^{-1}$. Individually these diagrams suffer from collinear divergence from region of integration where the momenta of the internal gravitons become parallel to that of the external graviton, but these divergences cancel in the sum over such graphs after using momentum conservation. Therefore we always work with sum of these diagrams. The net contribution from these diagrams receive logarithmic contribution from two regions - one where the loop momentum is large compared to $\omega$ and the other where the loop momentum is small compared to $\omega$. We shall analyze the contribution from the region of small loop momentum later. Contribution from the region where the loop 
momentum is large compared to $\omega$ may be approximated as

$$
\begin{aligned}
& -(i \lambda) \frac{i}{4} \sum_{\substack { a=1 \\
\begin{subarray}{c}{b=1 \\
b \neq a{ a = 1 \\
\begin{subarray} { c } { b = 1 \\
b \neq a } }\end{subarray}}^{n} \int_{\mathrm{reg}} \frac{d^{4} \ell}{(2 \pi)^{4}} \frac{1}{\left[p_{a} \cdot \ell-i \epsilon\right]\left[p_{b} \cdot \ell+i \epsilon\right]\left[\ell^{2}-i \epsilon\right]} \\
& {\left[-8\left(p_{a} \cdot \varepsilon \cdot p_{b}\right)\left(p_{a} \cdot p_{b}\right)+2\left(p_{a} \cdot \varepsilon \cdot p_{a}\right) p_{b}^{2}+2\left(p_{b} \cdot \varepsilon \cdot p_{b}\right) p_{a}^{2}-2\left\{2\left(p_{a} \cdot p_{b}\right)^{2}-p_{a}^{2} p_{b}^{2}\right\} \frac{\ell \cdot \varepsilon \cdot \ell}{\ell^{2}-i \epsilon}\right]} \\
& -(i \lambda) \frac{i}{2} \sum_{a=1}^{n} \int_{\mathrm{reg}} \frac{d^{4} \ell}{(2 \pi)^{4}} \frac{1}{\left[p_{a} \cdot \ell-i \epsilon\right]^{2}} \frac{1}{\left[\ell^{2}-i \epsilon\right]}\left[-2 p_{a}^{2}\left(p_{a} \cdot \varepsilon \cdot p_{a}\right)+\frac{\left(p_{a}^{2}\right)^{2}}{p_{a} \cdot \ell-i \epsilon}\left(p_{a} \cdot \varepsilon \cdot \ell\right)\right]
\end{aligned}
$$

In arriving at this result we have used integration by parts and also conservation of total momentum $\sum_{a=1}^{n} p_{a}=0$. We have also used the fact that in the expression for the graviton propagator carrying momentum $(k-\ell)$ in the second diagram of figure 11 , we can use the identity

$$
\frac{1}{(k-\ell)^{2}-i \epsilon}=\frac{2 \ell . k}{\left\{(k-\ell)^{2}-i \epsilon\right\}\left\{\ell^{2}-i \epsilon\right\}}+\frac{1}{\ell^{2}-i \epsilon}
$$

and ignore the contribution from the $\left(\ell^{2}-i \epsilon\right)^{-1}$ term, since the expression for the amplitude involving this term has no $k$-dependent denominator and therefore cannot have a $\ln \omega^{-1}$ term. ${ }^{11}$ Similar manipulations will be used in other terms as well.

Contribution to $\Gamma_{\text {self }}^{(n, 1)}$ given in figure 10 may be analyzed following the argument given below (5.16). We assume a general form $\varepsilon_{\mu \nu} p_{a}^{\mu} p_{a}^{\nu} f\left(p_{a} . k\right)$ for this amplitude based on Lorentz invariance and replace $\varepsilon_{\mu \nu}$ by $\xi_{\mu} k_{\nu}+\xi_{\nu} k_{\mu}$ for an arbitrary vector $\xi$ satisfying $k . \xi=0$. Then the amplitude reduces to $2 p_{a} \cdot \xi p_{a} \cdot k f\left(p_{a} \cdot k\right)$. On the other hand the diagrams in figure 10 for this choice of polarization may be evaluated using the Ward identity given in figure 15. Due to the presence of the non-vanishing right-hand side in figure 15, the result does not vanish. Comparing this with the expected result $2 p_{a} \cdot \xi p_{a} \cdot k f\left(p_{a} \cdot k\right)$, we can compute $f\left(p_{a} . k\right)$ and hence $\Gamma_{\text {self }}^{(n, 1)}$. It turns out that it receives logarithmic contribution from region of integration where the loop momentum is large compared to $\omega$. The result is:

$$
\Gamma_{\text {self }}^{(n, 1)}=-(i \lambda) \frac{i}{2} \sum_{a=1}^{n} p_{a}^{2} p_{a} \cdot \varepsilon \cdot p_{a} \int_{\text {reg }} \frac{d^{4} \ell}{(2 \pi)^{4}} \frac{1}{\left[p_{a} \cdot \ell-i \epsilon\right]^{2}\left[\ell^{2}-i \epsilon\right]} .
$$

This cancels the term in the last line of (6.18).

One loop contribution from the diagrams involving G-gravitons in figures 9 and 12 may be evaluated following the procedure described in section 5 . We find that the G-graviton contribution to figure 12 has no logarithmic contribution. Therefore we are left with the G-graviton contributions to figure 9. These diagrams have the same structure as in scalar QED and can be evaluated similarly. As in the case of scalar QED, these diagrams receive significant contribution only from the region where the loop momentum is large compared

\footnotetext{
${ }^{11}$ This manipulation can be carried out only for terms containing at least two powers of $\ell$ in the numerator so that each of the terms in (6.19) generates infrared finite integral.
} 
to $\omega$ and small compared to the momenta of finite energy particles. The net logarithmic contributions from these diagrams is given by

$$
\begin{aligned}
\Gamma_{G}^{(n, 1)}= & -(i \lambda) \frac{i}{2} \sum_{\substack { a=1 \\
\begin{subarray}{c}{b=1 \\
b \neq a{ a = 1 \\
\begin{subarray} { c } { b = 1 \\
b \neq a } }\end{subarray}}^{n} \int \frac{d^{4} l}{(2 \pi)^{4}} \frac{1}{\left[p_{a} \cdot l-i \epsilon\right]\left[p_{b} \cdot l+i \epsilon\right]\left[l^{2}-i \epsilon\right]} \\
& {\left[8\left(p_{a} \cdot p_{b}\right)\left(p_{a} \cdot \varepsilon \cdot p_{b}\right)-2 p_{b}^{2}\left(p_{a} \cdot \varepsilon \cdot p_{a}\right)-\left[2\left(p_{a} \cdot p_{b}\right)^{2}-p_{a}^{2} p_{b}^{2}\right]\left(\frac{p_{a} \cdot \varepsilon \cdot p_{a}}{p_{a}^{2}}+2 \frac{p_{a} \cdot \varepsilon \cdot l}{p_{a} \cdot l}\right)\right] } \\
& +(i \lambda) \frac{i}{2}\left(\frac{p_{a} \cdot \varepsilon \cdot p_{a}}{p_{a} \cdot k}\right) \int \frac{d^{4} l}{(2 \pi)^{4}} \frac{1}{\left[p_{a} \cdot l-i \epsilon\right]\left[p_{b} \cdot l+i \epsilon\right]\left[l^{2}-i \epsilon\right]} \\
& {\left[4\left(p_{a} \cdot p_{b}\right)\left(p_{b} \cdot k\right)-\left[2\left(p_{a} \cdot p_{b}\right)^{2}-p_{a}^{2} p_{b}^{2}\right] \frac{k \cdot l}{p_{a} \cdot l}\right] }
\end{aligned}
$$

The total logarithmic terms in $\Gamma_{\mathrm{G}}^{(n, 1)}, \Gamma_{\text {self }}^{(n, 1)}, \Gamma_{\text {residual }}^{(n, 1)}$ and $\Gamma_{3 \text {-graviton }}^{(n, 1)}$ from the region of integration where the loop momentum is large compared to $\omega$, can be expressed as ${ }^{12}$

$$
(i \lambda) \widehat{S}_{\mathrm{gr}}^{(1)} K_{\mathrm{gr}}^{\mathrm{reg}}
$$

where $\widehat{S}_{\mathrm{gr}}^{(1)}$ is the quantum subleading soft graviton operator

$$
\widehat{S}_{\mathrm{gr}}^{(1)}=\sum_{a} \frac{\varepsilon_{\mu \rho} p_{a}^{\rho} k_{\nu}}{p_{a} . k}\left\{p_{a}^{\mu} \frac{\partial}{\partial p_{a \nu}}-p_{a}^{\nu} \frac{\partial}{\partial p_{a \mu}}\right\},
$$

and

$$
K_{\mathrm{gr}}^{\mathrm{reg}} \equiv \frac{i}{2} \sum_{\substack{a, b \\ b \neq a}}\left\{\left(p_{a} \cdot p_{b}\right)^{2}-\frac{1}{2} p_{a}^{2} p_{b}^{2}\right\} \int_{\mathrm{reg}} \frac{d^{4} \ell}{(2 \pi)^{4}} \frac{1}{\ell^{2}-i \epsilon} \frac{1}{\left(p_{a} \cdot \ell-i \epsilon\right)\left(p_{b} \cdot \ell+i \epsilon\right)} .
$$

$K_{\mathrm{gr}}^{\mathrm{reg}}$ is the analog of $K_{\mathrm{em}}^{\mathrm{reg}}$ for gravitational scattering, namely it is the factor that appears in the exponent of the soft factor in the scattering of $n$ scalars, with the understanding that the integration over loop momentum is restricted to the region larger than $\omega$. We note however that the full expression for $K_{\mathrm{gr}}$ has more terms - (6.24) already involves an approximation that the loop momentum is small compared to the energies of external lines since this is the region that generates $\ln \omega^{-1}$ terms. Explicit evaluation gives the following expression for the terms involving $\ln \omega^{-1}$ :

$$
K_{\mathrm{gr}}^{\mathrm{reg}}=\frac{i}{2} \sum_{\substack{a, b \\ b \neq a}} \frac{1}{4 \pi} \ln \omega^{-1} \frac{\left\{\left(p_{a} \cdot p_{b}\right)^{2}-\frac{1}{2} p_{a}^{2} p_{b}^{2}\right\}}{\sqrt{\left(p_{a} \cdot p_{b}\right)^{2}-p_{a}^{2} p_{b}^{2}}}\left\{\delta_{\eta_{a} \eta_{b}, 1}-\frac{i}{2 \pi} \ln \left(\frac{p_{a} \cdot p_{b}+\sqrt{\left(p_{a} \cdot p_{b}\right)^{2}-p_{a}^{2} p_{b}^{2}}}{p_{a} \cdot p_{b}-\sqrt{\left(p_{a} \cdot p_{b}\right)^{2}-p_{a}^{2} p_{b}^{2}}}\right)\right\} .
$$

At this stage the only remaining terms are the contributions to $\Gamma_{3-\text { graviton }}^{(n, 1)}$ from regions of loop momentum integration where the loop momentum is small compared to $\omega$. These

\footnotetext{
${ }^{12}$ It is natural to conjecture that this pattern continues to hold also for subsubleading soft graviton theorem, i.e. the universal part of the subsubleading contribution is given by the action of the subsubleading soft graviton operator $\widehat{S}_{\mathrm{gr}}^{(2)}$ acting on $\exp \left[K_{\mathrm{gr}}^{\mathrm{reg}}\right]$. But we have not verified this by explicit computation.
} 
come from the first two diagrams in figure 11. In the first diagram there are two relevant regions: when $\ell$ is small and when $k-\ell$ is small, but they are related to each other by $\ell \rightarrow k-\ell$ and $a \leftrightarrow b$ symmetry. In the second diagram the relevant region is when $\ell$ is small. The net contribution from these regions may be approximated by

$$
\lambda \sum_{a=1}^{n} \sum_{b=1}^{n} \int \frac{d^{4} \ell}{(2 \pi)^{4}} \frac{1}{\left[2 k \cdot \ell+\ell^{2}+i \epsilon\right]\left[p_{a} \cdot \ell-i \epsilon\right]\left[\ell^{2}+i \epsilon\right]}\left[2\left(p_{a} \cdot \varepsilon \cdot p_{b}\right)\left(p_{a} \cdot k\right)-2\left(p_{b} \cdot \varepsilon \cdot p_{b}\right) \frac{\left(p_{a} \cdot k\right)^{2}}{p_{b} \cdot k}\right],
$$

with the understanding that the integration over $\ell$ runs in the region where the components of $\ell$ are small compared to $\omega$. The result may be expressed as

$$
i \lambda\left(\ln \omega^{-1}+\ln R^{-1}\right)\left[\frac{i}{4 \pi} \sum_{\substack{b \\ \eta_{b}=-1}} k \cdot p_{b} \sum_{a} \frac{\varepsilon_{\mu \nu} p_{a}^{\mu} p_{a}^{\nu}}{p_{a} \cdot k}-\frac{1}{8 \pi^{2}} \sum_{a} \frac{\varepsilon_{\mu \nu} p_{a}^{\mu} p_{a}^{\nu}}{p_{a} \cdot k} \sum_{b} p_{b} \cdot k \ln \frac{m_{b}^{2}}{\left(p_{b} . \hat{k}\right)^{2}}\right]
$$

where $1 / R$ is an infrared lower cut-off on momentum integration and $\hat{k}=-k / \omega=(1, \hat{n})$.

Adding (6.22) to (6.27) and dividing by $i \lambda$ we get the terms involving $\ln \omega^{-1}$ and $\ln R$ in $S_{\mathrm{gr}}^{(1)}$ :

$$
\begin{aligned}
S_{\mathrm{gr}}^{(1)}= & \widehat{S}_{g r}^{(1)} K_{\mathrm{gr}}^{\mathrm{reg}} \\
& +\frac{1}{4 \pi}\left(\ln \omega^{-1}+\ln R^{-1}\right)\left[i \sum_{\substack{b \\
\eta_{b}=-1}} k \cdot p_{b} \sum_{a} \frac{\varepsilon_{\mu \nu} p_{a}^{\mu} p_{a}^{\nu}}{p_{a} \cdot k}-\frac{1}{2 \pi} \sum_{a} \frac{\varepsilon_{\mu \nu} p_{a}^{\mu} p_{a}^{\nu}}{p_{a} \cdot k} \sum_{b} p_{b} \cdot k \ln \frac{m_{b}^{2}}{\left(p_{b} \cdot \hat{k}\right)^{2}}\right] .
\end{aligned}
$$

\section{Generalizations}

In this section we shall consider the case where the scalars interact via both electromagnetic and gravitational interaction via the action:

$$
\begin{gathered}
\int d^{4} x \sqrt{-\operatorname{det} g}\left[-\frac{1}{4} F_{\mu \nu} F^{\mu \nu}+\frac{1}{16 \pi G} R-\sum_{a=1}^{n}\left\{g^{\mu \nu}\left(\partial_{\mu} \phi_{a}^{*}+i q_{a} A_{\mu} \phi_{a}^{*}\right)\left(\partial_{\nu} \phi_{a}-i q_{a} A_{\nu} \phi_{a}\right)\right.\right. \\
\left.\left.+m_{a}^{2} \phi_{a}^{*} \phi_{a}\right\}+\lambda \phi_{1} \cdots \phi_{n}+\lambda \phi_{1}^{*} \cdots \phi_{n}^{*}\right]
\end{gathered}
$$

For this analysis we need two new vertices, the graviton-photon-photon vertex and the graviton-photon-scalar-scalar vertex. If the graviton carries an ingoing momentum $q$ and Lorentz index $(\rho \sigma)$, and the two photons carry ingoing momenta $k_{1}$ and $k_{2}$ and Lorentz indices $\mu$ and $\nu$ respectively, then the graviton-photon-photon vertex is given by:

$$
\begin{aligned}
& -i \kappa\left[\eta_{\rho \sigma}\left(-k_{1} \cdot k_{2} \eta_{\mu \nu}+k_{1 \nu} k_{2 \mu}\right)+\eta_{\mu \nu}\left(k_{1 \rho} k_{2 \sigma}+k_{2 \rho} k_{1 \sigma}\right)+k_{1} \cdot k_{2}\left(\eta_{\mu \rho} \eta_{\nu \sigma}+\eta_{\mu \sigma} \eta_{\nu \rho}\right)\right. \\
& \left.-\left(k_{1 \sigma} k_{2 \mu} \eta_{\rho \nu}+k_{2 \sigma} k_{1 \nu} \eta_{\rho \mu}+k_{1 \rho} k_{2 \mu} \eta_{\sigma \nu}+k_{2 \rho} k_{1 \nu} \eta_{\sigma \mu}\right)\right] .
\end{aligned}
$$


On the other hand the vertex with a pair of scalars carrying charges $q,-q$ and momenta $p_{1}$ and $p_{2}$, a graviton carrying Lorentz indices $(\mu \nu)$ and momentum $k_{1}$ and a photon carrying Lorentz index $\rho$ and momentum $k_{2}$, all counted ingoing, is given by

$$
-i \kappa q\left[\eta_{\mu \rho}\left(p_{1}-p_{2}\right)_{\nu}+\eta_{\nu \rho}\left(p_{1}-p_{2}\right)_{\mu}-\eta_{\mu \nu}\left(p_{1}-p_{2}\right)_{\rho}\right] \text {. }
$$

In this theory we shall analyze the extra terms in both the soft graviton theorem and the soft photon theorem.

There are two other vertices that are needed for our analysis. For example the sixth diagram of figure 18 needs the vertex containing two scalars, two photons and one graviton, whereas the sixth diagram of figure 23 requires the two scalar, two graviton and one photon vertex. However even without knowing the form of these vertices one can see that these diagrams do not generate contributions proportional to $\ln \omega^{-1}$. Therefore we have not written down the expressions for these vertices.

\subsection{Soft graviton theorem}

We first consider the soft graviton theorem. In this case besides the contributions analyzed in section 6 , we also have the diagrams of figure 17 and figure 18, obtained by replacing, in the diagrams in section 5 , the external photon by a graviton but keeping the internal line as a photon. We also have an additional set of diagrams shown in figure 19 where the external graviton connects to the internal photon. Diagrams in which the external graviton attaches to the $n$-scalar vertex vanish for $\varepsilon_{\rho}^{\rho}=0$ and have not been displayed. We carry out Grammer-Yennie decomposition for the internal photons in figure 17 following (5.3), but not for diagrams of the form shown in figure 18 and figure 19. The sum over K-photons factorize as in section 5 and gives the factor of $\exp \left[K_{\mathrm{em}}\right]$ that cancels between $\Gamma^{(n)}$ and $\Gamma^{(n, 1)}$. In this case there is no residual contribution since the analog of figure 4 holds with the upper photon in the second identity replaced by a graviton (see figure 20). This leads to the analog of (5.11) with an additional contribution to the left hand side given by diagrams of the form shown in figure 19. Denoting this contribution by $\Gamma_{\gamma \gamma g}^{(n, 1)}$ we arrive at the relation

$$
\Gamma_{\mathrm{self}}^{(n, 1)}+\Gamma_{\mathrm{G}}^{(n, 1)}+\Gamma_{\gamma \gamma g}^{(n, 1)}=i \lambda S_{\mathrm{gr}}^{(1)},
$$

with the understanding that both sides represent contributions in addition to what already appear in (6.16). None of the terms have any infrared divergence, and therefore there are no logarithmic terms from the region of integration in which the loop momentum is small compared to $\omega$. We shall describe below the organization of the various terms and then state the final result:

1. One can analyze $\Gamma_{\text {self }}^{(n, 1)}$ represented by the graphs in figure 18 by following the procedure described below (5.16). We replace the external graviton polarization by a pure gauge form $\left(\xi_{\mu} k_{\nu}+\xi_{\nu} k_{\mu}\right)$ and apply Ward identity to evaluate the sum over the graphs in figure 18. In this case the Ward identity has an additional contribution as shown in the right hand side of the second diagram in figure 21. It turns out however that its contribution to the amplitude does not have any logarithmic term. Therefore $\Gamma_{\text {self }}^{(n, 1)}$ does not generate any logarithmic contribution. 


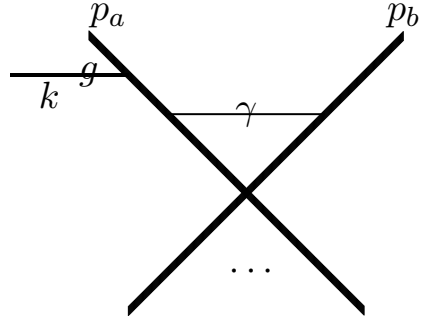

(a)



(b)

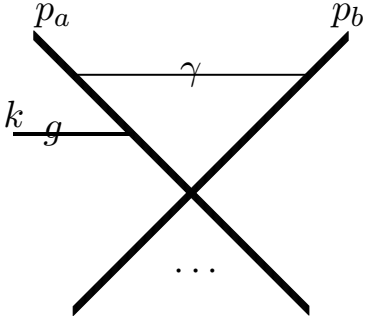

(c)

Figure 17. Contribution to soft graviton amplitude due to internal photon whose two ends are connected to two different scalar lines. Here the thickest lines denote scalars, lines of medium thickness carrying the symbol $g$ denote gravitons and the thin lines carrying the symbol $\gamma$ denote photons.
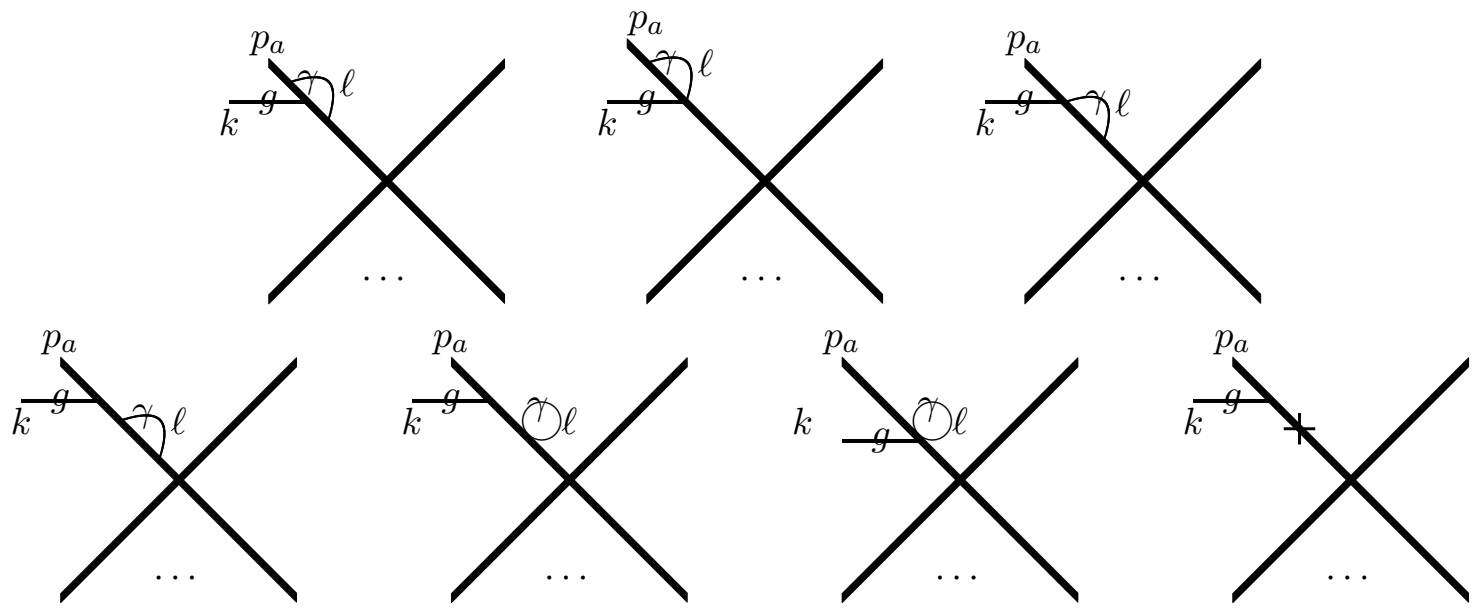

Figure 18. One loop contribution to soft graviton amplitude involving internal photon line connecting two points on the same leg.

2. $\Gamma_{\gamma \gamma g}^{(n, 1)}$ receives contribution proportional to $\ln \omega^{-1}$ from the first two diagrams of figure 19, from the region where the loop momentum is large compared to $\omega$.

3. Finally, the G-photon contribution $\Gamma_{\mathrm{G}}^{(n, 1)}$ from the first two diagrams in figure 17 also has terms proportional to $\ln \omega^{-1}$ from the region where the loop momentum is large compared to $\omega$.

The net logarithmic contribution from $\Gamma_{\gamma \gamma g}^{(n, 1)}$ and $\Gamma_{\mathrm{G}}^{(n, 1)}$ is given by:

$$
(i \lambda) \widehat{S}_{\mathrm{gr}}^{(1)} K_{\mathrm{em}}^{\mathrm{reg}} \text {. }
$$

After removing the $i \lambda$ factor, we have to add this to (6.28) to get the total logarithmic contribution to $S_{\mathrm{gr}}^{(1)}$ :

$$
\begin{aligned}
S_{\mathrm{gr}}^{(1)}= & \widehat{S}_{g r}^{(1)}\left(K_{\mathrm{em}}^{\mathrm{reg}}+K_{\mathrm{gr}}^{\mathrm{reg}}\right) \\
& +\frac{1}{4 \pi}\left(\ln \omega^{-1}+\ln R^{-1}\right)\left[i \sum_{\substack{b \\
\eta_{b}=-1}} k \cdot p_{b} \sum_{a} \frac{\varepsilon_{\mu \nu} p_{a}^{\mu} p_{a}^{\nu}}{p_{a} \cdot k}-\frac{1}{2 \pi} \sum_{a} \frac{\varepsilon_{\mu \nu} p_{a}^{\mu} p_{a}^{\nu}}{p_{a} \cdot k} \sum_{b} p_{b} \cdot k \ln \frac{m_{b}^{2}}{\left(p_{b} \cdot \hat{k}\right)^{2}}\right] .
\end{aligned}
$$



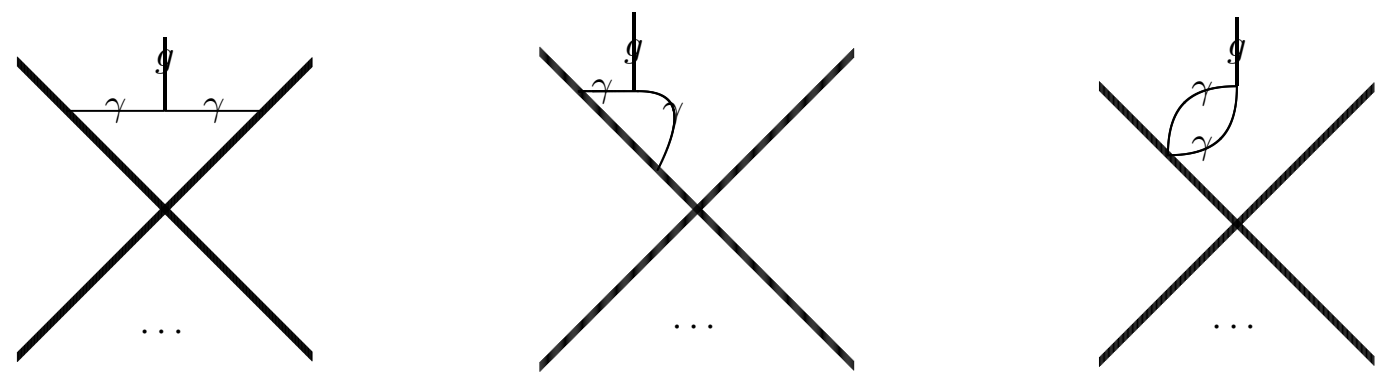

Figure 19. Diagrams containing graviton-photon-photon vertex that contribute to the soft photon contribution to the soft graviton theorem.



Figure 20. The Ward identity for the photon in the presence of a graviton-scalar-scalar vertex.
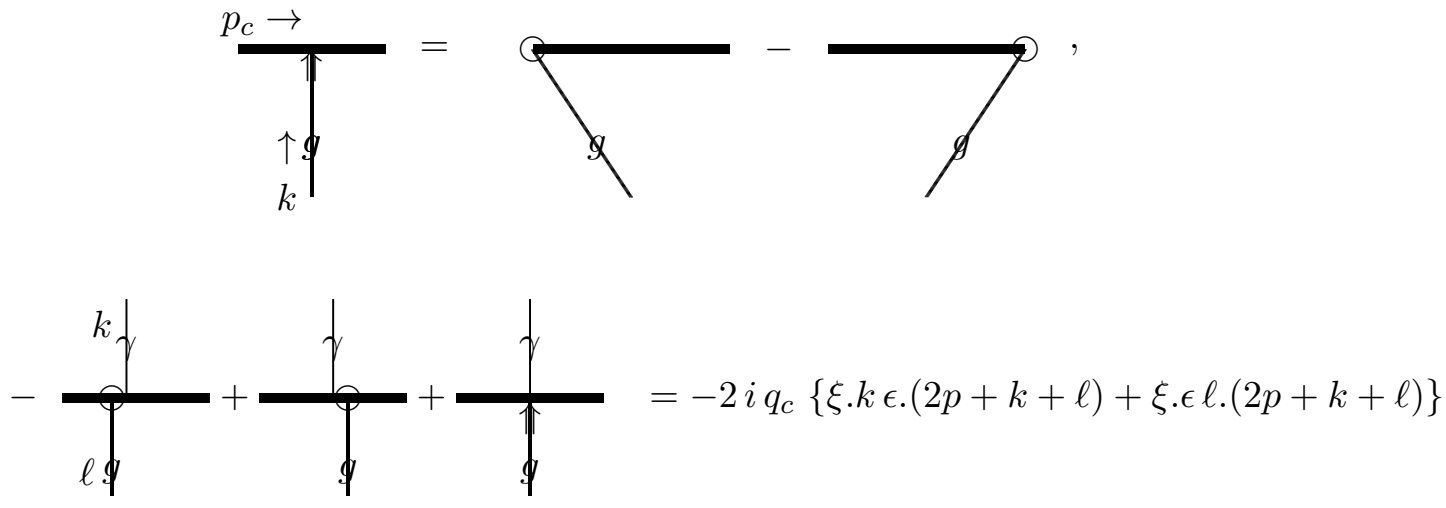

Figure 21. Analog of figure 4 for graviton in the presence of a photon. The graviton carries a polarization $\left(\xi_{\mu} \ell_{\nu}+\xi_{\nu} \ell_{\mu}\right)$ and the photon carries a polarization $\epsilon$. The circled vertex has been explained in the caption of figure 15 .

This reproduces terms proportional to $\ln \omega^{-1}$ in the sum of (2.6) and (2.7) after using (5.28) and (6.25).

\subsection{Soft photon theorem}

Next we shall consider the soft photon theorem. In this case we have all the diagrams considered in section 5, but also extra diagrams where the internal photon of figures 1 and 2 is replaced by an internal graviton, as shown in figures 22 and 23, and two additional sets of diagrams: one where one end of the internal graviton connects to the external photon as in figure 24 and the other where one end of the internal graviton is attached to the $n$-scalar vertex as in figure 25 . There is also an additional diagram obtained by 


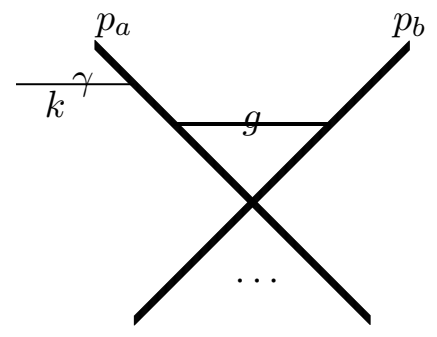

(a)

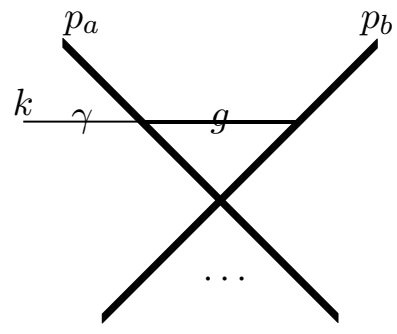

(b)



(c)

Figure 22. One loop contribution to soft photon amplitude involving internal graviton line connecting two different legs.
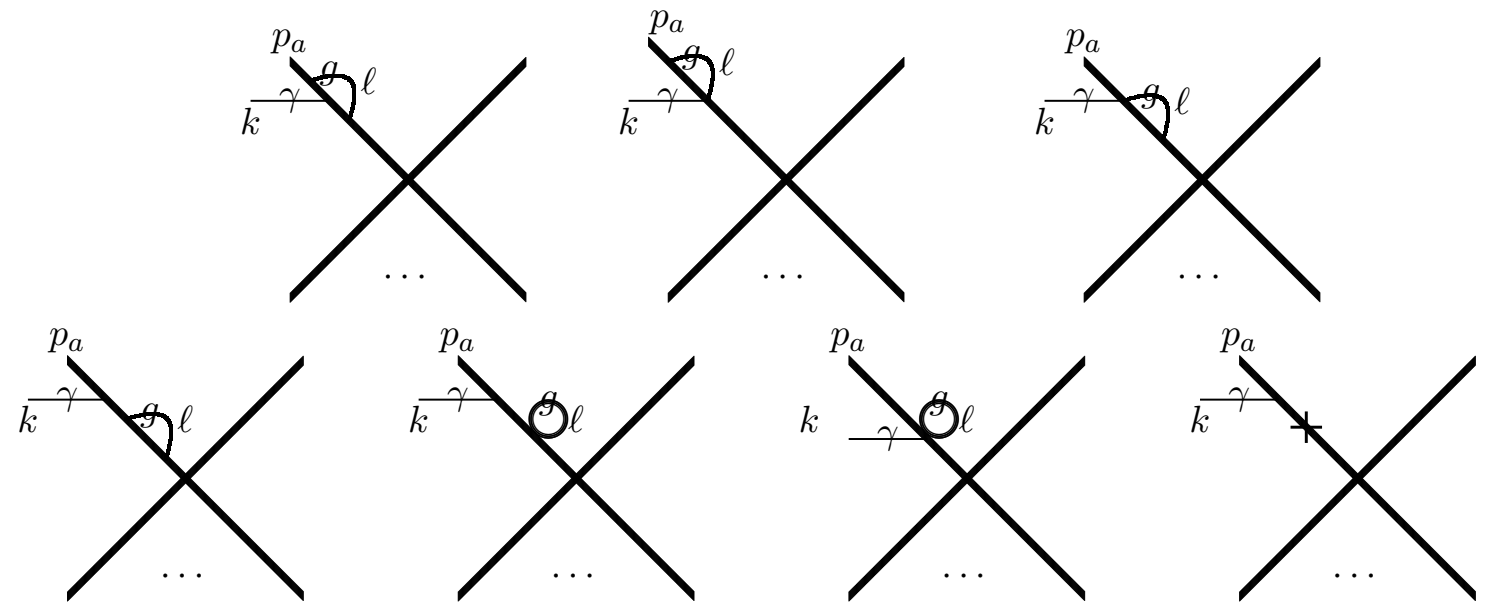

Figure 23. Diagrams in which the external photon and both ends of the internal graviton attach to the same scalar leg.
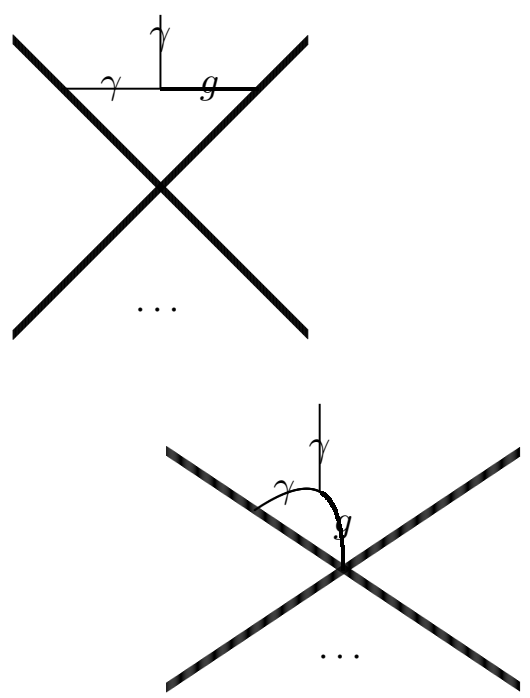
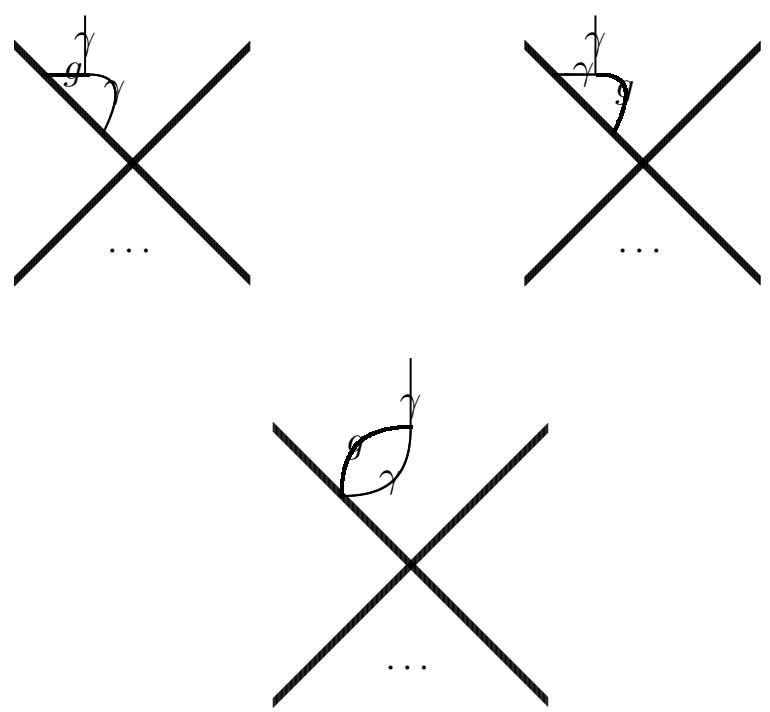

Figure 24. Diagrams involving graviton-photon-photon vertex that need to be included in computing the soft graviton contribution to the soft photon theorem. 

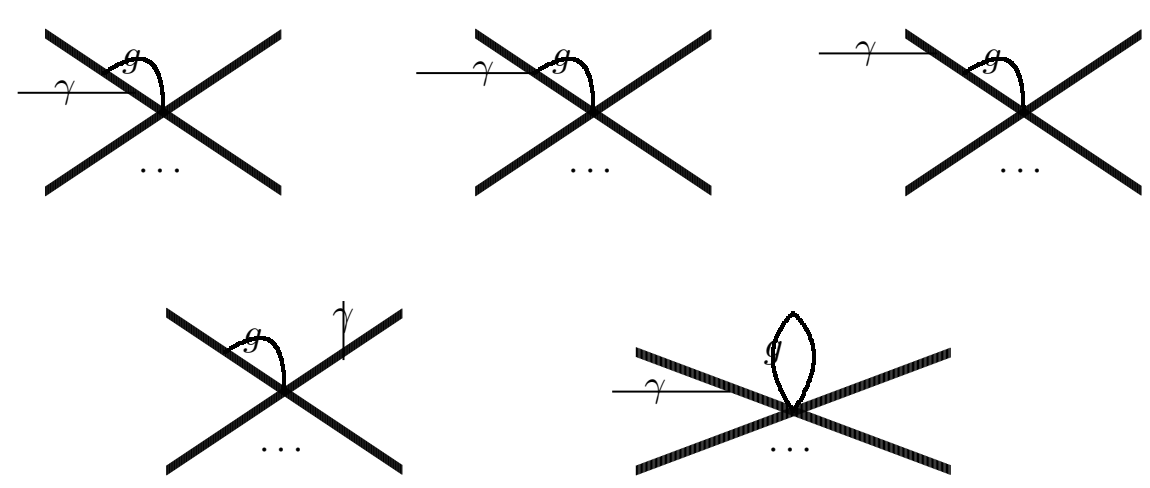

Figure 25. Diagrams with external soft photon and an internal graviton where the internal graviton attaches to the $n$-point vertex.

replacing in the first diagram of figure 14 the external graviton by the external photon, but this vanishes in dimensional regularization.

We shall analyze the diagrams in figures 22 and 25 using Grammer-Yennie decomposition for the internal graviton following the rules described in (6.9)-(6.14). The result of summing over K-gravitons in $\Gamma^{(n, 1)}$ will generate the factor of $\exp \left[K_{\mathrm{gr}}+\widetilde{K}_{\mathrm{gr}}\right]$ which cancels a similar factor in the expression of $\Gamma^{(n)}$. However there will be residual part that will be left over due to non-cancellation of the sum over K-graviton insertions reflected in the right-hand side of figure 21. Another residual contribution arises due to the momentum dependence of the circled vertices; as illustrated in figure 16, the factorized contribution of K-gravitons for $\Gamma^{(n)}$ and $\Gamma^{(n, 1)}$ differ. The only difference in the present case is that the external graviton carrying momentum $k$ in figure 16 is replaced by an external photon. As in section 6, we shall denote these residual contributions in the sum over K-gravitons by $\Gamma_{\text {residual }}^{(n, 1)}$. The G-graviton contribution to figures 22 and 25 will be denoted by $\Gamma_{\mathrm{G}}^{(n, 1)}$. The contribution from diagrams involving the coupling of graviton to photon, as shown in figure 24 , will be denoted by $\Gamma_{\gamma \gamma g}^{(n, 1)}$, and the contributions from figure 23 will be denoted by $\Gamma_{\text {self }}^{(n, 1)}$. Then the generalization of (5.11) takes the form:

$$
\Gamma_{\text {self }}^{(n, 1)}+\Gamma_{\mathrm{G}}^{(n, 1)}+\Gamma_{\gamma \gamma g}^{(n, 1)}+\Gamma_{\text {residual }}^{(n, 1)}=i \lambda S_{\mathrm{gr}}^{(1)},
$$

again with the understanding that both sides represent additional contribution besides those described in section 5 .

Analysis of various terms on the left hand side of (7.7) goes as follows:

1. $\Gamma_{\text {self }}^{(n, 1)}$ can be shown to vanish using the same argument given below (5.16). In this case the relevant Ward identities given in figures 4 and 20 do not have any left-over extra contributions.

2. It turns out that $\Gamma_{\text {residual }}^{(n, 1)}$, given by the left-over contribution after summing over $\mathrm{K}$-graviton insertions in figures 22 and 25, does not receive any logarithmic terms either from the region of loop momentum integration small compared to $\omega$ or from regions of loop momentum integration large compared to $\omega$. 
3. $\Gamma_{\mathrm{G}}^{(n, 1)}$ receives contributions proportional to $\ln \omega^{-1}$ only from the G-graviton contribution to figure 22 , from region of integration where the loop momentum is larger than $\omega$.

4. The individual diagrams contributing to $\Gamma_{\gamma \gamma g}^{(n, 1)}$ have collinear divergence from the region where the momenta of the internal graviton and photon are parallel to the momentum of the external photon. This cancels in the sum over all diagrams in figure 24. The second and third diagrams of figure 24 each has contribution proportional to $\ln \omega^{-1}$ from the region of integration where the loop momentum is large compared to $\omega$, but the sum of these contributions vanishes. Finally, $\Gamma_{\gamma \gamma g}^{(n, 1)}$ receives contributions proportional to $\ln \omega^{-1}$ from the first two diagrams in figure 24, from the region where the momentum of the internal graviton is smaller than $\omega$.

The net logarithmic contribution from the region of integration where the loop momentum is larger than $\omega$ is given by

$$
i \lambda \widehat{S}_{\mathrm{em}}^{(1)} K_{\mathrm{gr}}^{\mathrm{reg}}
$$

On the other hand the contribution to $\Gamma_{\gamma \gamma g}^{(n, 1)}$ from the small loop momentum region is given by:

$$
i \lambda\left(\ln \omega^{-1}+\ln R^{-1}\right)\left[\frac{i}{4 \pi} \sum_{\substack{b \\ \eta_{b}=-1}} k \cdot p_{b} \sum_{a} \frac{\varepsilon_{\mu} p_{a}^{\mu}}{p_{a} \cdot k} q_{a}-\frac{1}{8 \pi^{2}} \sum_{a=1}^{n} \frac{q_{a} \varepsilon_{\mu} p_{a}^{\mu}}{p_{a} \cdot k} \sum_{b=1}^{n}\left(p_{b} \cdot k\right) \ln \left(\frac{-p_{b}^{2}}{\left(p_{b} \cdot \hat{k}\right)^{2}}\right)\right] .
$$

One difference from the previous diagrams of this type, e.g. the ones shown in figure 11, is that the divergent contribution comes only from the region where the internal graviton momentum becomes small, and not when the internal photon momentum becomes small. This reflects the fact that while photons feel the long range gravitational force due to other particles, the graviton, being charge neutral, does not feel any long range Coulomb force. After removing the $i \lambda$ factors from (7.8) and (7.9), we have to add them to (5.20) to get the total soft factor $S_{\mathrm{em}}^{(1)}$. This gives

$$
\begin{aligned}
S_{\mathrm{em}}^{(1)} & =\widehat{S}_{\mathrm{em}}^{(1)}\left(K_{\mathrm{em}}^{\mathrm{reg}}+K_{\mathrm{gr}}^{\mathrm{reg}}\right) \\
& +\left(\ln \omega^{-1}+\ln R^{-1}\right)\left[\frac{i}{4 \pi} \sum_{\substack{b \\
\eta_{b}=-1}} k \cdot p_{b} \sum_{a} \frac{\varepsilon_{\mu} p_{a}^{\mu}}{p_{a} \cdot k} q_{a}-\frac{1}{8 \pi^{2}} \sum_{a=1}^{n} \frac{q_{a} \varepsilon_{\mu} p_{a}^{\mu}}{p_{a} \cdot k} \sum_{b=1}^{n}\left(p_{b} \cdot k\right) \ln \left(\frac{-p_{b}^{2}}{\left(p_{b} \cdot \hat{k}\right)^{2}}\right)\right]
\end{aligned}
$$

This reproduces terms proportional to $\ln \omega^{-1}$ in the sum of (2.4) and (2.5) after using the explicit forms of $K_{\mathrm{em}}^{\mathrm{reg}}$ and $K_{\mathrm{gr}}^{\mathrm{reg}}$ given in (5.28) and (6.25). 


\section{Acknowledgments}

We would like to thank Alok Laddha for many useful discussions and collaboration at the early stages of this work. We would also like to thank Massimo Bianchi and Gabriele Veneziano for discussions and the authors of $[97,98]$ for informing us of their results on the soft limit of scattering amplitudes of massless states. The work of A.S. was supported in part by the J.C. Bose fellowship of the Department of Science and Technology, India and also by the Infosys Chair Professorship.

Open Access. This article is distributed under the terms of the Creative Commons Attribution License (CC-BY 4.0), which permits any use, distribution and reproduction in any medium, provided the original author(s) and source are credited.

\section{References}

[1] M. Gell-Mann and M.L. Goldberger, Scattering of low-energy photons by particles of spin 1/2, Phys. Rev. 96 (1954) 1433 [InSPIRE].

[2] F.E. Low, Bremsstrahlung of very low-energy quanta in elementary particle collisions, Phys. Rev. 110 (1958) 974 [INSPIRE].

[3] S. Saito, Low-energy theorem for Compton scattering, Phys. Rev. 184 (1969) 1894 [INSPIRE].

[4] T.H. Burnett and N.M. Kroll, Extension of the low soft photon theorem, Phys. Rev. Lett. 20 (1968) 86 [INSPIRE].

[5] J.S. Bell and R. Van Royen, On the low-burnett-kroll theorem for soft-photon emission, Nuovo Cim. A 60 (1969) 62 [inSPIRE].

[6] V. Del Duca, High-energy Bremsstrahlung Theorems for Soft Photons, Nucl. Phys. B 345 (1990) 369 [INSPIRE].

[7] S. Weinberg, Photons and Gravitons in s Matrix Theory: Derivation of Charge Conservation and Equality of Gravitational and Inertial Mass, Phys. Rev. 135 (1964) B1049 [INSPIRE].

[8] S. Weinberg, Infrared photons and gravitons, Phys. Rev. 140 (1965) B516 [INSPIRE].

[9] D.J. Gross and R. Jackiw, Low-Energy Theorem for Graviton Scattering, Phys. Rev. 166 (1968) 1287 [INSPIRE].

[10] R. Jackiw, Low-Energy Theorems for Massless Bosons: Photons and Gravitons, Phys. Rev. 168 (1968) 1623 [INSPIRE].

[11] M. Ademollo et al., Soft Dilations and Scale Renormalization in Dual Theories, Nucl. Phys. B 94 (1975) 221 [INSPIRE].

[12] J.A. Shapiro, On the Renormalization of Dual Models, Phys. Rev. D 11 (1975) 2937 [INSPIRE].

[13] A. Strominger, On BMS Invariance of Gravitational Scattering, JHEP 07 (2014) 152 [arXiv: 1312.2229] [INSPIRE].

[14] T. He, V. Lysov, P. Mitra and A. Strominger, BMS supertranslations and Weinberg's soft graviton theorem, JHEP 05 (2015) 151 [arXiv:1401.7026] [INSPIRE].

[15] M. Campiglia and A. Laddha, Asymptotic symmetries and subleading soft graviton theorem, Phys. Rev. D 90 (2014) 124028 [arXiv:1408.2228] [INSPIRE]. 
[16] A. Strominger and A. Zhiboedov, Gravitational Memory, BMS Supertranslations and Soft Theorems, JHEP 01 (2016) 086 [arXiv:1411.5745] [INSPIRE].

[17] M. Campiglia and A. Laddha, New symmetries for the Gravitational S-matrix, JHEP 04 (2015) 076 [arXiv : 1502.02318] [INSPIRE].

[18] S. Pasterski, A. Strominger and A. Zhiboedov, New Gravitational Memories, JHEP 12 (2016) 053 [arXiv : 1502.06120] [INSPIRE].

[19] D. Kapec, V. Lysov, S. Pasterski and A. Strominger, Higher-Dimensional Supertranslations and Weinberg's Soft Graviton Theorem, arXiv:1502.07644 [INSPIRE].

[20] M. Campiglia and A. Laddha, Asymptotic symmetries of QED and Weinberg's soft photon theorem, JHEP 07 (2015) 115 [arXiv:1505.05346] [INSPIRE].

[21] S.G. Avery and B.U.W. Schwab, Burg-Metzner-Sachs symmetry, string theory and soft theorems, Phys. Rev. D 93 (2016) 026003 [arXiv: 1506.05789] [inSPIRE].

[22] M. Campiglia and A. Laddha, Asymptotic symmetries of gravity and soft theorems for massive particles, JHEP 12 (2015) 094 [arXiv: 1509.01406] [INSPIRE].

[23] M. Campiglia and A. Laddha, Sub-subleading soft gravitons: New symmetries of quantum gravity?, Phys. Lett. B 764 (2017) 218 [arXiv:1605.09094] [INSPIRE].

[24] M. Campiglia and A. Laddha, Subleading soft photons and large gauge transformations, JHEP 11 (2016) 012 [arXiv: 1605.09677] [INSPIRE].

[25] M. Campiglia and A. Laddha, Sub-subleading soft gravitons and large diffeomorphisms, JHEP 01 (2017) 036 [arXiv: 1608.00685] [INSPIRE].

[26] E. Conde and P. Mao, BMS Supertranslations and Not So Soft Gravitons, JHEP 05 (2017) 060 [arXiv: 1612.08294] [inSPIRE].

[27] T. He, D. Kapec, A.-M. Raclariu and A. Strominger, Loop-Corrected Virasoro Symmetry of 4D Quantum Gravity, JHEP 08 (2017) 050 [arXiv: 1701.00496] [INSPIRE].

[28] M. Asorey, A.P. Balachandran, F. Lizzi and G. Marmo, Equations of Motion as Constraints: Superselection Rules, Ward Identities, JHEP 03 (2017) 136 [arXiv:1612.05886] [INSPIRE].

[29] A. Campoleoni, D. Francia and C. Heissenberg, On higher-spin supertranslations and superrotations, JHEP 05 (2017) 120 [arXiv:1703.01351] [INSPIRE].

[30] A. Strominger, Lectures on the Infrared Structure of Gravity and Gauge Theory, arXiv: 1703.05448 [INSPIRE].

[31] M. Pate, A.-M. Raclariu and A. Strominger, Color Memory: A Yang-Mills Analog of Gravitational Wave Memory, Phys. Rev. Lett. 119 (2017) 261602 [arXiv:1707.08016] [INSPIRE].

[32] A. Laddha and P. Mitra, Asymptotic Symmetries and Subleading Soft Photon Theorem in Effective Field Theories, JHEP 05 (2018) 132 [arXiv:1709.03850] [INSPIRE].

[33] D. Kapec and P. Mitra, A d-Dimensional Stress Tensor for Mink ${ }_{d+2}$ Gravity, JHEP 05 (2018) 186 [arXiv: 1711.04371] [INSPIRE].

[34] M. Pate, A.-M. Raclariu and A. Strominger, Gravitational Memory in Higher Dimensions, JHEP 06 (2018) 138 [arXiv: 1712.01204] [INSPIRE].

[35] A. Campoleoni, D. Francia and C. Heissenberg, Asymptotic Charges at Null Infinity in Any Dimension, Universe 4 (2018) 47 [arXiv:1712.09591] [InSPIRE]. 
[36] A.H. Anupam, A. Kundu and K. Ray, Double soft graviton theorems and Bondi-Metzner-Sachs symmetries, Phys. Rev. D 97 (2018) 106019 [arXiv:1803.03023] [INSPIRE].

[37] C.D. White, Factorization Properties of Soft Graviton Amplitudes, JHEP 05 (2011) 060 [arXiv:1103.2981] [INSPIRE].

[38] F. Cachazo and A. Strominger, Evidence for a New Soft Graviton Theorem, arXiv: 1404.4091 [INSPIRE].

[39] E. Casali, Soft sub-leading divergences in Yang-Mills amplitudes, JHEP 08 (2014) 077 [arXiv: 1404.5551] [INSPIRE].

[40] B.U.W. Schwab and A. Volovich, Subleading Soft Theorem in Arbitrary Dimensions from Scattering Equations, Phys. Rev. Lett. 113 (2014) 101601 [arXiv:1404.7749] [inSPIRE].

[41] Z. Bern, S. Davies and J. Nohle, On Loop Corrections to Subleading Soft Behavior of Gluons and Gravitons, Phys. Rev. D 90 (2014) 085015 [arXiv:1405.1015] [INSPIRE].

[42] S. He, Y.-t. Huang and C. Wen, Loop Corrections to Soft Theorems in Gauge Theories and Gravity, JHEP 12 (2014) 115 [arXiv:1405.1410] [INSPIRE].

[43] A.J. Larkoski, Conformal Invariance of the Subleading Soft Theorem in Gauge Theory, Phys. Rev. D 90 (2014) 087701 [arXiv:1405.2346] [INSPIRE].

[44] F. Cachazo and E.Y. Yuan, Are Soft Theorems Renormalized?, arXiv:1405.3413 [INSPIRE].

[45] N. Afkhami-Jeddi, Soft Graviton Theorem in Arbitrary Dimensions, arXiv:1405.3533 [INSPIRE].

[46] B.U.W. Schwab, Subleading Soft Factor for String Disk Amplitudes, JHEP 08 (2014) 062 [arXiv: 1406.4172] [INSPIRE].

[47] M. Bianchi, S. He, Y.-t. Huang and C. Wen, More on Soft Theorems: Trees, Loops and Strings, Phys. Rev. D 92 (2015) 065022 [arXiv:1406.5155] [INSPIRE].

[48] J. Broedel, M. de Leeuw, J. Plefka and M. Rosso, Constraining subleading soft gluon and graviton theorems, Phys. Rev. D 90 (2014) 065024 [arXiv:1406.6574] [INSPIRE].

[49] Z. Bern, S. Davies, P. Di Vecchia and J. Nohle, Low-Energy Behavior of Gluons and Gravitons from Gauge Invariance, Phys. Rev. D 90 (2014) 084035 [arXiv:1406.6987] [INSPIRE].

[50] C.D. White, Diagrammatic insights into next-to-soft corrections, Phys. Lett. B 737 (2014) 216 [arXiv: 1406.7184] [INSPIRE].

[51] M. Zlotnikov, Sub-sub-leading soft-graviton theorem in arbitrary dimension, JHEP 10 (2014) 148 [arXiv: 1407.5936] [INSPIRE].

[52] C. Kalousios and F. Rojas, Next to subleading soft-graviton theorem in arbitrary dimensions, JHEP 01 (2015) 107 [arXiv:1407.5982] [INSPIRE].

[53] Y.-J. Du, B. Feng, C.-H. Fu and Y. Wang, Note on Soft Graviton theorem by KLT Relation, JHEP 11 (2014) 090 [arXiv: 1408.4179] [InSPIRE].

[54] D. Bonocore, E. Laenen, L. Magnea, L. Vernazza and C.D. White, The method of regions and next-to-soft corrections in Drell-Yan production, Phys. Lett. B 742 (2015) 375 [arXiv: 1410.6406] [INSPIRE]. 
[55] B.U.W. Schwab, A Note on Soft Factors for Closed String Scattering, JHEP 03 (2015) 140 [arXiv: 1411.6661] [INSPIRE].

[56] A. Sabio Vera and M.A. Vazquez-Mozo, The Double Copy Structure of Soft Gravitons, JHEP 03 (2015) 070 [arXiv: 1412.3699] [inSPIRE].

[57] P. Di Vecchia, R. Marotta and M. Mojaza, Soft theorem for the graviton, dilaton and the Kalb-Ramond field in the bosonic string, JHEP 05 (2015) 137 [arXiv:1502.05258] [INSPIRE].

[58] F. Cachazo, S. He and E.Y. Yuan, New Double Soft Emission Theorems, Phys. Rev. D 92 (2015) 065030 [arXiv:1503.04816] [INSPIRE].

[59] A.E. Lipstein, Soft Theorems from Conformal Field Theory, JHEP 06 (2015) 166 [arXiv: 1504.01364] [INSPIRE].

[60] T. Klose, T. McLoughlin, D. Nandan, J. Plefka and G. Travaglini, Double-Soft Limits of Gluons and Gravitons, JHEP 07 (2015) 135 [arXiv:1504.05558] [INSPIRE].

[61] A. Volovich, C. Wen and M. Zlotnikov, Double Soft Theorems in Gauge and String Theories, JHEP 07 (2015) 095 [arXiv: 1504.05559] [INSPIRE].

[62] M. Bianchi and A.L. Guerrieri, On the soft limit of open string disk amplitudes with massive states, JHEP 09 (2015) 164 [arXiv: 1505.05854] [INSPIRE].

[63] P. Di Vecchia, R. Marotta and M. Mojaza, Double-soft behavior for scalars and gluons from string theory, JHEP 12 (2015) 150 [arXiv:1507.00938] [INSPIRE].

[64] A.L. Guerrieri, Soft behavior of string amplitudes with external massive states, Nuovo Cim. C 39 (2016) 221 [arXiv:1507.08829] [InSPIRE].

[65] S.D. Alston, D.C. Dunbar and W.B. Perkins, n-point amplitudes with a single negative-helicity graviton, Phys. Rev. D 92 (2015) 065024 [arXiv:1507.08882] [INSPIRE].

[66] Y.-t. Huang and C. Wen, Soft theorems from anomalous symmetries, JHEP 12 (2015) 143 [arXiv: 1509.07840] [INSPIRE].

[67] P. Di Vecchia, R. Marotta and M. Mojaza, Soft Theorems from String Theory, Fortsch. Phys. 64 (2016) 389 [arXiv:1511.04921] [INSPIRE].

[68] M. Bianchi and A.L. Guerrieri, On the soft limit of closed string amplitudes with massive states, Nucl. Phys. B 905 (2016) 188 [arXiv:1512.00803] [INSPIRE].

[69] M. Bianchi and A.L. Guerrieri, On the soft limit of tree-level string amplitudes, in Proceedings, 14th Marcel Grossmann Meeting on Recent Developments in Theoretical and Experimental General Relativity, Astrophysics and Relativistic Field Theories (MG14) (In 4 Volumes), Rome, Italy, July 12-18, 2015, vol. 4, pp. 4157-4163 (2017) [DOI:10.1142/9789813226609_0555] [arXiv:1601.03457] [INSPIRE].

[70] J. Rao and B. Feng, Note on Identities Inspired by New Soft Theorems, JHEP 04 (2016) 173 [arXiv: 1604.00650] [INSPIRE].

[71] P. Di Vecchia, R. Marotta and M. Mojaza, Subsubleading soft theorems of gravitons and dilatons in the bosonic string, JHEP 06 (2016) 054 [arXiv: 1604.03355] [INSPIRE].

[72] S. He, Z. Liu and J.-B. Wu, Scattering Equations, Twistor-string Formulas and Double-soft Limits in Four Dimensions, JHEP 07 (2016) 060 [arXiv: 1604.02834] [INSPIRE].

[73] F. Cachazo, P. Cha and S. Mizera, Extensions of Theories from Soft Limits, JHEP 06 (2016) 170 [arXiv: 1604.03893] [INSPIRE]. 
[74] A.P. Saha, Double Soft Theorem for Perturbative Gravity, JHEP 09 (2016) 165 [arXiv: 1607.02700] [INSPIRE].

[75] P. Di Vecchia, R. Marotta and M. Mojaza, Soft behavior of a closed massless state in superstring and universality in the soft behavior of the dilaton, JHEP 12 (2016) 020 [arXiv: 1610.03481] [INSPIRE].

[76] A. Luna, S. Melville, S.G. Naculich and C.D. White, Next-to-soft corrections to high energy scattering in QCD and gravity, JHEP 01 (2017) 052 [arXiv: 1611.02172] [INSPIRE].

[77] H. Elvang, C.R.T. Jones and S.G. Naculich, Soft Photon and Graviton Theorems in Effective Field Theory, Phys. Rev. Lett. 118 (2017) 231601 [arXiv:1611.07534] [INSPIRE].

[78] C. Cheung, K. Kampf, J. Novotny, C.-H. Shen and J. Trnka, A Periodic Table of Effective Field Theories, JHEP 02 (2017) 020 [arXiv:1611.03137] [INSPIRE].

[79] A.P. Saha, Double soft limit of the graviton amplitude from the Cachazo-He-Yuan formalism, Phys. Rev. D 96 (2017) 045002 [arXiv:1702.02350] [InSPIRE].

[80] A. Sen, Soft Theorems in Superstring Theory, JHEP 06 (2017) 113 [arXiv:1702.03934] [INSPIRE].

[81] A. Sen, Subleading Soft Graviton Theorem for Loop Amplitudes, JHEP 11 (2017) 123 [arXiv:1703.00024] [INSPIRE].

[82] P. Di Vecchia, R. Marotta and M. Mojaza, Double-soft behavior of the dilaton of spontaneously broken conformal invariance, JHEP 09 (2017) 001 [arXiv:1705.06175] [INSPIRE].

[83] A. Laddha and A. Sen, Sub-subleading Soft Graviton Theorem in Generic Theories of Quantum Gravity, JHEP 10 (2017) 065 [arXiv:1706.00759] [INSPIRE].

[84] S. Chakrabarti, S.P. Kashyap, B. Sahoo, A. Sen and M. Verma, Subleading Soft Theorem for Multiple Soft Gravitons, JHEP 12 (2017) 150 [arXiv:1707.06803] [INSPIRE].

[85] Y. Hamada and G. Shiu, Infinite Set of Soft Theorems in Gauge-Gravity Theories as Ward-Takahashi Identities, Phys. Rev. Lett. 120 (2018) 201601 [arXiv:1801.05528] [INSPIRE].

[86] Z.-Z. Li, H.-H. Lin and S.-Q. Zhang, Infinite Soft Theorems from Gauge Symmetry, Phys. Rev. D 98 (2018) 045004 [arXiv: 1802.03148] [INSPIRE].

[87] S. Higuchi and H. Kawai, Universality of soft theorem from locality of soft vertex operators, Nucl. Phys. B 936 (2018) 400 [arXiv: 1805.11079] [INSPIRE].

[88] A. Laddha and A. Sen, Gravity Waves from Soft Theorem in General Dimensions, JHEP 09 (2018) 105 [arXiv: 1801.07719] [INSPIRE].

[89] A. Laddha and A. Sen, Logarithmic Terms in the Soft Expansion in Four Dimensions, JHEP 10 (2018) 056 [arXiv: 1804.09193] [INSPIRE].

[90] A. Laddha and A. Sen, Observational Signature of the Logarithmic Terms in the Soft Graviton Theorem, arXiv: 1806.01872 [INSPIRE].

[91] P.C. Peters, Relativistic gravitational bremsstrahlung, Phys. Rev. D 1 (1970) 1559 [INSPIRE].

[92] L. Blanchet and T. Damour, Hereditary effects in gravitational radiation, Phys. Rev. D 46 (1992) 4304 [INSPIRE]. 
[93] W.D. Goldberger and A. Ross, Gravitational radiative corrections from effective field theory, Phys. Rev. D 81 (2010) 124015 [arXiv:0912.4254] [InSPIRE].

[94] W.D. Goldberger, A. Ross and I.Z. Rothstein, Black hole mass dynamics and renormalization group evolution, Phys. Rev. D 89 (2014) 124033 [arXiv:1211.6095] [INSPIRE].

[95] A. Gruzinov and G. Veneziano, Gravitational Radiation from Massless Particle Collisions, Class. Quant. Grav. 33 (2016) 125012 [arXiv:1409.4555] [INSPIRE].

[96] M. Ciafaloni, D. Colferai, F. Coradeschi and G. Veneziano, Unified limiting form of graviton radiation at extreme energies, Phys. Rev. D 93 (2016) 044052 [arXiv:1512.00281] [INSPIRE].

[97] A. Addazi, M. Bianchi and G. Veneziano, Soft gravitational radiation from ultra-relativistic collisions at sub- and sub-sub-leading order, arXiv:1901.10986 [INSPIRE].

[98] M. Ciafaloni, D. Colferai and G. Veneziano, Infrared features of gravitational scattering and radiation in the eikonal approach, arXiv:1812.08137 [INSPIRE].

[99] G. Grammer Jr. and D.R. Yennie, Improved treatment for the infrared divergence problem in quantum electrodynamics, Phys. Rev. D 8 (1973) 4332 [InSPIRE]. 